\title{
Archimedean Muirhead Aggregation Operators of q-Rung Orthopair Fuzzy Numbers for Multicriteria Group Decision Making
}

\author{
Yuchu Qin, ${ }^{1}$ Xiaolan Cui, ${ }^{2}$ Meifa Huang ${ }^{D},{ }^{1}$ Yanru Zhong, ${ }^{3}$ Zhemin Tang, ${ }^{1}$ and Peizhi Shi ${ }^{4}$ \\ ${ }^{1}$ School of Mechanical and Electrical Engineering, Guilin University of Electronic Technology, Guilin 541004, China \\ ${ }^{2}$ School of Management, Huazhong University of Science and Technology, Wuhan 430074, China \\ ${ }^{3}$ Guangxi Key Laboratory of Intelligent Processing of Computer Images and Graphic, Guilin University of Electronic Technology, \\ Guilin 541004, China \\ ${ }^{4}$ School of Computing and Engineering, University of Huddersfield, Huddersfield HD1 3DH, UK \\ Correspondence should be addressed to Meifa Huang; meifahuang@yeah.net
}

Received 9 June 2019; Revised 9 August 2019; Accepted 24 August 2019; Published 17 December 2019

Academic Editor: Mohammad Hassan Khooban

Copyright (C) 2019 Yuchu Qin et al. This is an open access article distributed under the Creative Commons Attribution License, which permits unrestricted use, distribution, and reproduction in any medium, provided the original work is properly cited.

\begin{abstract}
q-Rung orthopair fuzzy number (qROFN) is a flexible and superior fuzzy information description tool which can provide stronger expressiveness than intuitionistic fuzzy number and Pythagorean fuzzy number. Muirhead mean (MM) operator and its dual form geometric MM (GMM) operator are two all-in-one aggregation operators for capturing the interrelationships of the aggregated arguments because they are applicable in the cases in which all arguments are independent of each other, there are interrelationships between any two arguments, and there are interrelationships among any three or more arguments. Archimedean T-norm and T-conorm (ATT) are superior operations that can generate general and versatile operational rules to aggregate arguments. To take advantage of qROFN, MM operator, GMM operator, and ATT in multicriteria group decision making (MCGDM), an Archimedean MM operator, a weighted Archimedean MM operator, an Archimedean GMM operator, and a weighted Archimedean GMM operator for aggregating qROFNs are presented to solve the MCGDM problems based on qROFNs in this paper. The properties of these operators are explored and their specific cases are discussed. On the basis of the presented operators, a method for solving the MCGDM problems based on qROFNs is proposed. The effectiveness of the proposed method is demonstrated via a numerical example, a set of experiments, and qualitative and quantitative comparisons. The demonstration results suggest that the proposed method has satisfying generality and flexibility at aggregating q-rung orthopair fuzzy information and capturing the interrelationships of criteria and the attitudes of decision makers and is feasible and effective for solving the MCGDM problems based on qROFNs.
\end{abstract}

\section{Introduction}

Multicriteria group decision making (MCGDM) or multicriteria group decision analysis is a process of finding optimal alternatives in complex scenarios via synthetically evaluating the values of multiple criteria of all alternatives from a group of experts [1]. In this process, one of the fundamental tasks is to accurately and effectively describe the values of multiple criteria. For such description, there are various available mathematical tools, where fuzzy set is one of the most important and popular tools. To date, over twenty different types of fuzzy sets have been presented within academia [2]. Among the presented fuzzy sets, Zadeh's fuzzy set (FS) [3] is a well-known type of fuzzy set that quantifies the satisfaction degree via a membership degree whose range is $[0,1]$. The expressiveness of FS is stronger enough for expressing the fuzzy information in some applications. However, FS is incapable of describing the complex fuzzy information that contains the dissatisfaction and hesitancy degrees.

To describe complex fuzzy information, Atanassov [4] presented the intuitionistic fuzzy set (IFS) theory. In this 
theory, an IFS has a membership degree and a nonmembership degree (both degrees and their sum are restricted to $[0,1])$, which can, respectively, quantify the satisfaction and dissatisfaction degrees, and the hesitancy degree is naturally obtained using one minus the sum of the membership and nonmembership degrees. Because of such expressiveness, IFSs have been widely used to express the values of multiple criteria in MCGDM during the past few decades. A variety of research topics about IFSs for MCGDM, such as operational rules of intuitionistic fuzzy numbers (IFNs) [5, 6], aggregation operators of IFNs [7-9], intuitionistic preference relations $[10,11]$, intuitionistic fuzzy calculus $[12,13]$, and multicriteria decision making (MCDM) or MCGDM methods based on IFSs [14-16], have become hot topics in the research of MCGDM in this period.

Although IFSs have gained importance and popularity in the field of MCGDM, their application is still limited by their expressiveness. To be more specific, the sum of the membership and nonmembership degrees must be in $[0,1]$ in IFSs. But in some practical applications, there may be some values of criteria whose membership and nonmembership degrees' sum is greater than one. Such values cannot be described via IFSs. To solve this issue, Yager [17] proposed the Pythagorean fuzzy set (PFS) theory, in which the condition is relaxed to the following: the sum of squares of the membership and nonmembership degrees is restricted to [0, 1]. As a result, the values whose membership and nonmembership degrees' sum is greater than one could be expressed using PFSs. Because of stronger expressiveness than IFSs, PFSs have also achieved a wide range of applications in MCGDM. A number of research topics regarding PFSs for MCGDM, such as operational rules of Pythagorean fuzzy numbers (PFNs) $[18,19]$, correlation and correlation coefficient of PFSs [20], information measures of PFSs [21-23], aggregation operators of PFNs [24-27], and MCDM or MCGDM methods based on PFSs [28-30], have received widespread attention during the past few years.

To further improve the expressiveness of PFSs, Yager [31] presented the q-rung orthopair fuzzy set (qROFS) theory, in which the condition is further relaxed to the following: the sum of the $q$-th $(q=1,2,3, \ldots)$ power of the membership degree and the $q$-th power of the nonmembership degree is in $[0,1]$. From this condition, it is not difficult to find that qROFS is actually the generalization of IFS and PFS since qROFS will reduce to IFS when $q=1$ and will reduce to PFS when $q=2$. In addition, it can also be found that as the value of the rung $q$ increases, the expressiveness of qROFSs will continue to increase, which provides more freedom for the quantification of fuzzy information. Due to such characteristic, qROFSs have also received extensive attention in the field of MCGDM during the past few years. Various research topics about qROFSs for MCGDM, such as operational rules of q-rung orthopair fuzzy numbers (qROFNs) (i.e., q-rung orthopair membership grades) [32, 33], approximate reasoning in qROFSs [34], aspects of qROFSs [35], correlation coefficient between qROFSs [36], distance measures of qROFSs [37], and aggregation operators of qROFNs [38-50], are gaining importance and popularity within academia.
For solving MCGDM problems, there are usually two ways. One way is to leverage traditional methods (e.g., TOPSIS, VIKOR, TODIM, ELECTRE, and PROMETHEE), and the other way is to use aggregation operators [51-53]. In general, aggregation operators can solve MCGDM problems more effectively than traditional methods because they can generate comprehensive values and rankings of alternatives, while traditional methods can only provide rankings [42]. So far, over twenty different aggregation operators of qROFNs have been presented. Representative examples are the weighted exponential (WE) operator presented by Peng et al. [38], the weighted point (WP) operators presented by Xing et al. [39], the weighted averaging (WA) operator and weighted geometric (WG) operator presented by Liu and Wang [40], the weighted Bonferroni mean (WBM) operator and weighted geometric Bonferroni mean (WGBM) operator presented by Liu and Liu [41], the weighted Archimedean Bonferroni mean (WABM) operators presented by Liu and Wang [42], the weighted extended Bonferroni mean (WEBM) operator presented by Liu et al. [43], the weighted partitioned Bonferroni mean (WPBM) operator and weighted partitioned geometric Bonferroni mean (WPGBM) operator presented by Yang and Pang [44], the weighted Heronian mean (WHM) operator and weighted geometric Heronian mean (WGHM) operator presented by Wei et al. [45], the WHM* operator (please note that this operator is different from the WHM operator in [45] although they have the same names) and weighted partitioned Heronian mean (WPHM) operator presented by Liu et al. [46], the weighted Maclaurin symmetric mean (WMSM) operator and weighted geometric Maclaurin symmetric mean (WGMSM) operator presented by Wei et al. [47], the weighted power Maclaurin symmetric mean (WPMSM) operator presented by Liu et al. [48], the weighted power partitioned Maclaurin symmetric mean (WPPMSM) operator presented by Bai et al. [49], and the weighted Muirhead mean (WMM) operator and weighted geometric Muirhead mean (WGMM) operator presented by Wang et al. [50]. Each operator has its own features and application conditions. However, there is not yet an operator that is both versatile and flexible for capturing the interrelationships of criteria and the attitudes of decision makers when solving the MCGDM problems based on qROFNs.

In actual MCGDM problems, the interrelationships of criteria and the attitudes of decision makers are likely to change as the actual situation changes. An ideal aggregation operator should provide desirable generality and flexibility to adapt to these changes [42]. Among the aggregation operators of qROFNs above, the WE, WP, WA, and WG operators are only applicable for the qROFNs based MCGDM problems where all criteria are independent of each other. The WBM, WGBM, WABM, WEBM, WPBM, WPGBM, WHM, WGHM, WHM*, and WPHM operators are more general in capturing the interrelationships of criteria than the WE, WP, WA, and WG operators. They can be used to solve the problems in which there are no interrelationships among criteria or there are interrelationships between two criteria. The WMSM, WGMSM, WPMSM, WPPMSM, WMM, and WGMM operators are 
the most versatile in dealing with the interrelationships. They are applicable for the problems where all criteria are mutually independent or there are interrelationships between two or among more criteria. But their flexibility in reflecting the attitudes of decision makers is limited because the aggregations in these operators are based on the fixed Algebraic T-norm and T-conorm operation. Based on this, the motivations of this paper are summarized as follows:

(1) To develop aggregation operators of qROFNs that are versatile in capturing the interrelationships of criteria, the Muirhead mean (MM) and geometric MM (GMM) operators [54] are introduced. The MM and GMM operators, which are the generalizations of the generalized arithmetic averaging, generalized geometric averaging, Bonferroni mean (BM), geometric BM (GBM), Maclaurin symmetric mean (MSM), and geometric MSM (GMSM) operators, are two all-in-one aggregation operators for dealing with the interrelationships of criteria because they are suitable for the cases in which all criteria are independent of each other, there are interrelationships between any two criteria, and there are interrelationships among any multiple (more than two) criteria $[50,55,56]$.

(2) To improve the generality and flexibility in reflecting decision makers' attitudes of the aggregation operators, the Archimedean T-norm and T-conorm (ATT) operations [57] are adopted to perform the operations in them. The ATT operations are important mathematical tools for constructing general operational rules for fuzzy numbers. A fuzzy information aggregation operator based on them is rather versatile and flexible for capturing the attitudes of decision makers [42].

Based on the motivations above, the present paper combines the MM and GMM operators with the ATT operations under q-rung orthopair fuzzy environment to construct Archimedean MM and GMM operators of qROFNs for the MCGDM problems based on qROFNs. Due to such combination, the constructed operators can achieve satisfying generality and flexibility in capturing both the interrelationships of criteria and the attitudes of decision makers. The major contributions of the paper are as follows: (1) a q-rung orthopair fuzzy Archimedean MM (qROFAMM) operator and a q-rung orthopair fuzzy weighted Archimedean MM (qROFWAMM) operator are presented; (2) a q-rung orthopair fuzzy Archimedean GMM (qROFAGMM) operator and a q-rung orthopair fuzzy weighted Archimedean GMM (qROFWAGMM) operator are presented; and (3) a MCGDM method based on the presented operators is developed. Although there are already two Muirhead aggregation operators of qROFNs (i.e., the WMM and WGMM operators in [50]), the presented operators are still of necessity because they are more general and flexible than these two operators. The WMM and WGMM operators are based on the Algebraic T-norm and T-conorm, one of the many families of ATTs, while the Archimedean MM and
GMM operators can be applied to any families of ATTs and the WMM and WGMM operators are just their special cases, respectively.

The remainder of the paper is organized as follows. A brief introduction of some related fundamental concepts is provided Section 2. Sections 3, respectively, explains the details of the presented Archimedean MM and GMM operators. A MCGDM method based on the Archimedean MM and GMM operators is designed in Section 4. Section 5 demonstrates and evaluates the presented operators and designed method via a numerical example, a set of experiments, and qualitative and quantitative comparisons. Section 6 ends the paper with a conclusion.

\section{Preliminaries}

In this section, some prerequisites in the qROFS theory, operational rules of qROFNs based on ATT, and MM and GMM operators are briefly introduced to facilitate the understanding of the present paper.

2.1. qROFS Theory. qROFS [31] is the generalization of FS [3], IFS [4], and PFS [17]. Its formal definition is as follows.

Definition 1 (see [31]). A qROFS $S$ in a finite universe of discourse $X$ is $S=\left\{<x, \mu_{S}(x), v_{S}(x)>\mid x \in X\right\}$, where $\mu_{S}: X \longrightarrow[0$, $1]$ is the membership degree of $x \in X$ to $S$ and $\nu_{S}: X \longrightarrow[0,1]$ is the nonmembership degree of $x \in X$ to $S$, such that $0 \leq\left(\mu_{S}(x)\right)^{q}+\left(\nu_{S}(x)\right)^{q} \leq 1 \quad(q=1,2,3, \ldots)$. The hesitancy degree of $x \in X$ to $S$ is $\pi_{S}(x)=\left(1-\left(\mu_{S}(x)\right)^{q}-\left(v_{S}(x)\right)^{q}\right)^{1 / q}$.

For convenience, a pair $\left\langle\mu_{S}(x), \nu_{S}(x)>\right.$ is called a qROFN, which is commonly simplified as $\Xi=\langle\mu, \nu\rangle$. To compare two qROFNs, their scores and accuracies are required, which can be calculated according to the following definitions.

Definition 2 (see [40]). Let $\Xi=\langle\mu, v\rangle$ be a qROFN. Then its score is $S(\Xi)=\mu^{q}-\gamma^{q}$. Obviously, $-1 \leq S(\Xi) \leq 1$.

Definition 3 (see [40]). Let $\Xi=\langle\mu, v\rangle$ be a qROFN. Then its accuracy is $A(\Xi)=\mu^{q}+\nu^{q}$. Obviously, $0 \leq A(\Xi) \leq 1$.

Using $S(\Xi)$ and $A(\Xi)$, two qROFNs can be compared via the following definition.

Definition 4 (see [40]). Let $\Xi_{1}=\left\langle\mu_{1}, \nu_{1}\right\rangle$ and $\Xi_{2}=\left\langle\mu_{2}, \nu_{2}\right\rangle$ be any two qROFNs, $S\left(\Xi_{1}\right)$ and $S\left(\Xi_{2}\right)$ be, respectively, the scores of $\Xi_{1}$ and $\Xi_{2}$, and $A\left(\Xi_{1}\right)$ and $A\left(\Xi_{2}\right)$ be, respectively, the accuracies of $\Xi_{1}$ and $\Xi_{2}$. Then, (1) if $S\left(\Xi_{1}\right)>S\left(\Xi_{2}\right)$, then $\Xi_{1}>\Xi_{2}$; (2) if $S\left(\Xi_{1}\right)=S\left(\Xi_{2}\right)$ and $A\left(\Xi_{1}\right)>A\left(\Xi_{2}\right)$, then $\Xi_{1}>\Xi_{2}$; and (3) if $S\left(\Xi_{1}\right)=S\left(\Xi_{2}\right)$ and $A\left(\Xi_{1}\right)=A\left(\Xi_{2}\right)$, then $\Xi_{1}=\Xi_{2}$.

2.2. Operational Rules of qROFNs Based on ATT. Based on ATT, a set of general and flexible operational rules of qROFNs were presented in [42], which can be formally defined as follows.

Definition 5 (see [42]). Let $\Xi=\langle\mu, \nu\rangle, \Xi_{1}=\left\langle\mu_{1}, \nu_{1}\right\rangle$, and $\left.\Xi_{2}=<\mu_{2}, \nu_{2}\right\rangle$ be any three qROFNs, and $\sigma$ and $\tau$ be any two 
real numbers and $\sigma, \tau>0$. Then the sum, product, multiplication, and power operations of qROFNs based on the Archimedean T-norm $T(x, y)=f^{-1}(f(x)+f(y))$ and its T-conorm $T^{\mathrm{C}}(x, y)=g^{-1}(g(x)+g(y))$ can be, respectively, defined as follows:

$$
\begin{aligned}
\Xi_{1} \oplus \Xi_{2} & =\left\langle T^{C}\left(\mu_{1}, \mu_{2}\right), T\left(\nu_{1}, v_{2}\right)\right\rangle \\
& =\left\langle g^{-1}\left(g\left(\mu_{1}\right)+g\left(\mu_{2}\right)\right), f^{-1}\left(f\left(v_{1}\right)+f\left(v_{2}\right)\right)\right\rangle,
\end{aligned}
$$

$$
\begin{aligned}
\Xi_{1} \otimes \Xi_{2} & =\left\langle T\left(\mu_{1}, \mu_{2}\right), T^{C}\left(v_{1}, v_{2}\right)\right\rangle \\
& =\left\langle f^{-1}\left(f\left(\mu_{1}\right)+f\left(\mu_{2}\right)\right), g^{-1}\left(g\left(v_{1}\right)+g\left(v_{2}\right)\right)\right\rangle,
\end{aligned}
$$

$$
\begin{gathered}
\sigma \Xi=\left\langle g^{-1}(\sigma g(\mu)), f^{-1}(\sigma f(\nu))\right\rangle, \\
\Xi^{\tau}=\left\langle f^{-1}(\tau f(\mu)), g^{-1}(\tau g(\nu))\right\rangle .
\end{gathered}
$$

2.3. MM and GMM Operators. The MM operator was introduced to aggregate crisp numbers by Muirhead [54]. It has prominent characteristics in capturing the interrelationships among multiple aggregated arguments and providing a general form of a number of other aggregation operators. The formal definition of the MM operator is as follows.

Definition 6 (see [54]). Let $\left(\Theta_{1}, \Theta_{2}, \ldots, \Theta_{n}\right)$ be a collection of crisp numbers, $\Delta=\left(\delta_{1}, \delta_{2}, \ldots, \delta_{n}\right)$ (where $\delta_{1}, \delta_{2}, \ldots, \delta_{n} \geq 0$ but not at the same time $\left.\delta_{1}=\delta_{2}=\ldots=\delta_{n}=0\right)$ be a collection of $n$ real numbers, $p(i)$ be a permutation of $(1,2, \ldots, n)$, and $\mathbf{P}_{n}$ be the set of all permutations of $(1,2, \ldots, n)$. Then the aggregation function,

$$
\operatorname{MM}^{\Delta}\left(\Theta_{1}, \Theta_{2}, \ldots, \Theta_{n}\right)=\left(\frac{1}{n !} \sum_{p \in \mathbf{P}_{n}} \prod_{i=1}^{n} \Theta_{p(i)}^{\delta_{i}}\right)^{1 / \Sigma_{i=1}^{n} \delta_{i}},
$$

is called the MM operator.

In this operator, whether the interrelationships are considered depends on the values of $\delta_{i}(i=1,2, \ldots, n)$ : (1) if $\delta_{1}>0$ and $\delta_{2}=\delta_{3}=\ldots=\delta_{n}=0$, then the interrelationships are not considered; (2) if $\delta_{1}, \delta_{2}>0$ and $\delta_{3}=\delta_{4}=\ldots=\delta_{n}=0$, then the interrelationships between two crisp numbers are considered; and (3) if $\delta_{1}, \delta_{2}, \ldots, \delta_{k}>0(k=3,4, \ldots, n)$ and $\delta_{k+1}=\delta_{k+2}=\ldots=\delta_{n}=0$, then the interrelationships among $k$ crisp numbers are considered.

The dual form of the MM operator is called the dual MM or GMM operator. Its formal definition is as follows.

Definition 7 (see [55]). Let $\left(\Theta_{1}, \Theta_{2}, \ldots, \Theta_{n}\right)$ be a collection of crisp numbers, $\Delta=\left(\delta_{1}, \delta_{2}, \ldots, \delta_{n}\right)$ (where $\delta_{1}, \delta_{2}, \ldots, \delta_{n} \geq 0$ but not at the same time $\delta_{1}=\delta_{2}=\ldots=\delta_{n}=0$ ) be a collection of $n$ real numbers, $p(i)$ be a permutation of $(1,2, \ldots, n)$, and $\mathbf{P}_{n}$ be the set of all permutations of $(1,2, \ldots, n)$. Then, the aggregation function,

$$
\operatorname{GMM}^{\Delta}\left(\Theta_{1}, \Theta_{2}, \ldots, \Theta_{n}\right)=\frac{1}{\sum_{i=1}^{n} \delta_{i}}\left(\prod_{p \in \mathbf{P}_{n}} \sum_{i=1}^{n}\left(\delta_{i} \Theta_{p(i)}\right)\right)^{1 / n !},
$$

is called the GMM operator.

Similarly, in this operator, whether the interrelationships are described also relies on the values of $\delta_{i}(i=1,2, \ldots, n)$ with the same cases as they are in the MM operator.

\section{Archimedean Muirhead Aggregation Operators}

This section consists of two subsections. In the first subsection, a qROFAMM operator and a qROFWAMM operator are presented using the $\mathrm{MM}$ operator and the operational rules of qROFNs based on ATT. The properties of these two operators are proved and their specific cases are discussed. In the second subsection, the dual form of the qROFAMM operator, i.e., a qROFAGMM operator, and the dual form of the qROFWAMM operator, i.e., a qROFWAGMM operator, are presented using the GMM operator and the operational rules of qROFNs based on ATT. The properties of these two operators are explored and their specific cases are discussed.

\subsection{Archimedean MM Operators}

3.1.1. qROFAMM Operator. A qROFAMM operator is a MM operator for aggregating qROFNs, in which the sum, product, multiplication, and power operations are performed using the operational rules of qROFNs based on ATT. Its formal definition is as follows.

Definition 8. Let $\left(\Xi_{1}, \Xi_{2}, \ldots, \Xi_{n}\right)\left(\Xi_{i}=<\mu_{i}, \nu_{i}>, i=1,2, \ldots, n\right)$ be a collection of $n$ qROFNs $(q=1,2,3, \ldots), \Delta=\left(\delta_{1}, \delta_{2}, \ldots\right.$, $\left.\delta_{n}\right) \quad\left(\delta_{1}, \delta_{2}, \ldots, \delta_{n} \geq 0\right.$ but not at the same time $\left.\delta_{1}=\delta_{2}=\ldots=\delta_{n}=0\right)$ be a collection of $n$ real numbers, $p(i)$ be a permutation of $(1,2, \ldots, n), \mathbf{P}_{n}$ be the set of all permutations of $(1,2, \ldots, n), \Xi_{i} \oplus \Xi_{j}$ and $\Xi_{i} \otimes \Xi_{j}(i, j=1,2, \ldots, n)$ be, respectively, the sum and product operations of $\Xi_{i}$ and $\Xi_{j}$ based on ATT, and $\sigma \Xi_{r}$ and $\Xi_{s}^{\tau}(r, s=1,2, \ldots, n ; \sigma, \tau>0)$ be, respectively, the multiplication operation of $\Xi_{r}$ and the power operation of $\Xi_{s}$ based on ATT. Then, the aggregation function,

$$
\operatorname{qROFAMM}^{\Delta}\left(\Xi_{1}, \Xi_{2}, \ldots, \Xi_{n}\right)=\left(\frac{1}{n !} \underset{p \in \mathbf{P}_{n}}{\oplus} \otimes_{i=1}^{n}\left(\Xi_{p(i)}^{\delta_{i}}\right)\right)^{1 / \Sigma_{i=1}^{n} \delta_{i}},
$$

is called the qROFAMM operator.

According to equations (1)-(4) and (7), the following theorem is obtained.

Theorem 1. Let $\left(\Xi_{1}, \Xi_{2}, \ldots, \Xi_{n}\right)\left(\Xi_{i}=<\mu_{i}, v_{i}>, i=1,2, \ldots, n\right)$ be a collection of $n$ RROFNs $(q=1,2,3, \ldots)$. Then 


$$
\begin{aligned}
\operatorname{qROFAMM}^{\Delta}\left(\Xi_{1}, \Xi_{2}, \ldots, \Xi_{n}\right)= & \left\langlef ^ { - 1 } \left(\frac{1}{\sum_{i=1}^{n} \delta_{i}} f\left(g^{-1}\left(\frac{1}{n !} \sum_{p \in \mathbf{P}_{n}} g\left(f^{-1}\left(\sum_{i=1}^{n}\left(\delta_{i} f\left(\mu_{p(i)}\right)\right)\right)\right)\right)\right),\right.\right. \\
& \left.g^{-1}\left(\frac{1}{\sum_{i=1}^{n} \delta_{i}} g\left(f^{-1}\left(\frac{1}{n !} \sum_{p \in \mathbf{P}_{n}} f\left(g^{-1}\left(\sum_{i=1}^{n}\left(\delta_{i} g\left(v_{p(i)}\right)\right)\right)\right)\right)\right)\right)\right\rangle
\end{aligned}
$$

and $q \operatorname{ROFAMM} M^{\Delta}\left(\Xi_{1}, \Xi_{2}, \ldots, \Xi_{n}\right)$ is still a $q R O F N$.

For the details of the proof of this theorem, please refer to Appendix A. The following three theorems, respectively, state the idempotency, monotonicity, and boundedness of the qROFAMM operator:

Theorem 2 (idempotency). Let $\left(\Xi_{1}, \Xi_{2}, \ldots, \Xi_{n}\right)\left(\Xi_{i}=\left\langle\mu_{i}, v_{i}\right\rangle\right.$, $i=1,2, \ldots, n)$ be a collection of $n q R O F N s(q=1,2,3, \ldots)$. If $\Xi_{i}=\Xi=\left\langle\mu, v>\right.$ for all $i=1,2, \ldots, n$, then $q \operatorname{ROFAMM}^{\Delta}\left(\Xi_{1}, \Xi_{2}\right.$, $\left.\ldots, \Xi_{n}\right)=\Xi$.

Theorem 3 (monotonicity). Let $\left(\Xi_{1,1}, \Xi_{1,2}, \ldots, \Xi_{1, n}\right)$ $\left(\Xi_{1, i}=\left\langle\mu_{1, i}, v_{1, i}>, i=1,2, \ldots, n\right)\right.$ and $\left(\Xi_{2,1}, \Xi_{2,2}, \ldots, \Xi_{2, n}\right)$ $\left(\Xi_{2, i}=\left\langle\mu_{2, i}, v_{2, i}>\right)\right.$ be two collections of $n q R O F N s(q=1,2,3$, ...). If $\mu_{1, i} \geq \mu_{2, i}$ and $v_{1, i} \leq v_{2, i}$ for all $i=1,2, \ldots, n$, then $\operatorname{qROFAMM}^{\Delta}\left(\Xi_{1,1}, \Xi_{1,2}, \ldots, \Xi_{1, n}\right) \geq q \operatorname{ROFAMM}^{\Delta}\left(\Xi_{2,1}, \Xi_{2,2}\right.$, $\left.\ldots, \Xi_{2, n}\right)$.

Theorem 4 (boundedness). Let $\left(\Xi_{1}, \Xi_{2}, \ldots, \Xi_{n}\right)\left(\Xi_{i}=<\mu_{i}\right.$, $\left.v_{i}>, i=1,2, \ldots, n\right)$ be a collection of $n q R O F N s(q=1,2,3$, $\left.\ldots), \quad \Xi_{U B}=<\max \left(\mu_{i}\right), \quad \min \left(v_{i}\right)\right\rangle$, and $\Xi_{L B}=<\min \left(\mu_{i}\right)$, $\max \left(v_{i}\right)>$. Then $\Xi_{L B} \leq q R \operatorname{ROFAM} M^{\Delta}\left(\Xi_{1}, \Xi_{2}, \ldots, \Xi_{n}\right) \leq \Xi_{U B}$.

For the details of the proofs of these three theorems, please refer to Appendixes B-D, respectively.

Equation (8) is a generalized form of the qROFAMM operator. If specific values are assigned to $q$ and $\delta_{1}, \delta_{2}, \ldots, \delta_{n}$ and specific forms are assigned to $f$, then specific operators can be obtained:

(1) If $q=1$, then the qROFAMM operator will reduce to an intuitionistic fuzzy Archimedean MM operator.

(2) If $q=2$, then the qROFAMM operator will reduce to a Pythagorean fuzzy Archimedean MM operator.

(3) If $\delta_{1}=\delta>0$ and $\delta_{2}=\delta_{3}=\ldots=\delta_{n}=0$, then the qROFAMM operator will reduce to a q-rung orthopair fuzzy Archimedean generalized arithmetic averaging operator.

(4) If $\delta_{1}, \delta_{2}>0$ and $\delta_{3}=\delta_{4}=\ldots=\delta_{n}=0$, then the qROFAMM operator will reduce to the q-rung orthopair fuzzy Archimedean BM operator presented by Liu and Wang [42].

(5) If $\delta_{1}=\delta_{2}=\ldots=\delta_{k}=1$ and $\delta_{k+1}=\delta_{k+2}=\ldots=\delta_{n}=0$, then the qROFAMM operator will reduce to a q-rung orthopair fuzzy Archimedean MSM operator.

(6) If $\delta_{1}=\delta_{2}=\ldots=\delta_{n}=\delta>0$, then the qROFAMM operator will reduce to a q-rung orthopair fuzzy
Archimedean generalized geometric averaging operator.

(7) If $f(t)=-\operatorname{In} t^{q}$, then $g(t)=-\operatorname{In}\left(1-t^{q}\right), f^{-1}(t)=\left(e^{-t}\right)^{1 / q}$, and $g^{-1}(t)=\left(1-e^{-t}\right)^{1 / q}$. According to equation (8), a q-rung orthopair fuzzy Archimedean Algebraic MM (qROFAAMM) operator is constructed as follows:

$$
\begin{aligned}
& \operatorname{qROFAAMM}^{\Delta}\left(\Xi_{1}, \Xi_{2}, \ldots, \Xi_{n}\right) \\
& =\left\langle\left(\left(1-\left(\prod_{p \in \mathbf{P}_{n}}\left(1-\prod_{i=1}^{n} \mu_{p(i)}^{q \delta_{i}}\right)\right)^{1 / n !}\right)^{1 / \sum_{i=1}^{n} \delta_{i}}\right)^{1 / q},\right. \\
& \left.\left(1-\left(1-\left(\prod_{p \in \mathbf{P}_{n}}\left(1-\prod_{i=1}^{n}\left(1-v_{p(i)}^{q}\right)^{\delta_{i}}\right)\right)^{1 / n !}\right)^{1 / \Sigma_{i=1}^{n} \delta_{i}}\right)^{1 / q}\right\rangle .
\end{aligned}
$$

This operator is actually the q-rung orthopair fuzzy MM operator presented by Wang et al. [50]. It has the following special cases:

(1) If $q=1$, then the qROFAAMM operator will reduce to

$$
\begin{aligned}
& \left\langle\left(1-\left(\prod_{p \in \mathbf{P}_{n}}\left(1-\prod_{i=1}^{n} \mu_{p(i)}^{\delta_{i}}\right)\right)^{1 / n !}\right)^{1 / \sum_{i=1}^{n} \delta_{i}},\right. \\
& \left.1-\left(1-\left(\prod_{p \in \mathbf{P}_{n}}\left(1-\prod_{i=1}^{n}\left(1-v_{p(i)}\right)^{\delta_{i}}\right)\right)^{1 / n !}\right)^{1 / \Sigma_{i=1}^{n} \delta_{i}}\right\rangle,
\end{aligned}
$$

which is the intuitionistic fuzzy MM operator presented by Liu and $\mathrm{Li}$ [55].

(2) If $q=2$, then the qROFAAMM operator will reduce to

$$
\begin{aligned}
& \left\langle\left(\left(1-\left(\prod_{p \in \mathbf{P}_{n}}\left(1-\prod_{i=1}^{n} \mu_{p(i)}^{2 \delta_{i}}\right)\right)^{1 / n !}\right)^{1 / \Sigma_{i=1}^{n} \delta_{i}}\right)^{1 / 2},\right. \\
& \left.\left(1-\left(1-\left(\prod_{p \in \mathbf{P}_{n}}\left(1-\prod_{i=1}^{n}\left(1-v_{p(i)}^{2}\right)^{\delta_{i}}\right)\right)^{1 / n !}\right)^{1 / \Sigma_{i=1}^{n} \delta_{i}}\right)^{1 / 2}\right\rangle,
\end{aligned}
$$

which is the Pythagorean fuzzy MM operator presented by Zhu and Li [56].

(3) If $\delta_{1}=\delta>0$ and $\delta_{2}=\delta_{3}=\ldots=\delta_{n}=0$, then the qROFAAMM operator will reduce to 


$$
\left\langle\left(\left(1-\left(\prod_{i=1}^{n}\left(1-\mu_{i}^{q \delta}\right)\right)^{1 / n}\right)^{1 / \delta}\right)^{1 / q},\left(1-\left(1-\left(\prod_{i=1}^{n}\left(1-\left(1-v_{i}^{q}\right)^{\delta}\right)\right)^{1 / n}\right)^{1 / \delta}\right)^{1 / q}\right\rangle
$$

which is a q-rung orthopair fuzzy generalized arithmetic averaging operator.
(4) If $\delta_{1}, \delta_{2}>0$ and $\delta_{3}=\delta_{4}=\ldots=\delta_{n}=0$, then the qROFAAMM operator will reduce to

$$
\left\langle\left(\left(1-\left(\prod_{\substack{i, j=1 \\ j \neq i}}^{n}\left(1-\mu_{i}^{q \delta_{1}} \mu_{j}^{q \delta_{2}}\right)\right)^{1 / n(n-1)}\right)^{1 /\left(\delta_{1}+\delta_{2}\right)}\right)^{1 / q},\left(1-\left(1-\left(\prod_{\substack{i, j=1 \\ j \neq i}}^{n}\left(1-\left(1-v_{i}^{q}\right)^{\delta_{1}}\left(1-v_{j}^{q}\right)^{\delta_{2}}\right)\right)^{1 / n(n-1)}\right)^{1 /\left(\delta_{1}+\delta_{2}\right)}\right)^{1 / q}\right)^{1 / q}
$$

which is the q-rung orthopair fuzzy Archimedean Algebraic BM operator presented by Liu and Wang [42].
(5) If $\delta_{1}=\delta_{2}=\ldots=\delta_{k}=1$ and $\delta_{k+1}=\delta_{k+2}=\ldots=$ $\delta_{n}=0$, then the qROFAAMM operator will reduce to

$$
\left\langle\left(\left(1-\prod_{1 \leq i_{1}<\ldots<i_{k} \leq n}^{n}\left(1-\prod_{j=1}^{k} \mu_{i_{j}}^{q}\right)^{k !(n-k) ! / n !}\right)^{1 / k}\right)^{1 / q},\left(1-\left(1-\prod_{1 \leq i_{1}<\ldots<i_{k} \leq n}^{n}\left(1-\prod_{j=1}^{k}\left(1-v_{i_{j}}^{q}\right)\right)^{k !(n-k) ! / n !}\right)^{1 / k}\right)^{1 / q}\right\rangle^{\prime}
$$

which is the q-rung orthopair fuzzy MSM operator presented by Wei et al. [47] and Liu et al. [48].

(6) If $\delta_{1}=\delta_{2}=\ldots=\delta_{n}=\delta>0$, then the qROFAAMM operator will reduce to

$$
\begin{aligned}
& \left\langle\left(1-\left(1-\prod_{i=1}^{n}\left(1-\left(1-\mu_{i}^{q}\right)^{\delta}\right)^{1 / n}\right)^{1 / \delta}\right)^{1 / q},\right. \\
& \left.\left(\left(1-\prod_{i=1}^{n}\left(1-v_{i}^{q \delta}\right)^{1 / n}\right)^{1 / \delta}\right)^{1 / q}\right\rangle,
\end{aligned}
$$

which is a q-rung orthopair fuzzy generalized geometric averaging operator.

(7) If $f(t)=\operatorname{In}\left[\left(2-t^{q}\right) / t^{q}\right]$, then $g(t)=\operatorname{In}\left[\left(1+t^{q}\right) /\right.$ $\left.\left(1-t^{q}\right)\right], \quad f^{-1}(t)=\left[2 /\left(\mathrm{e}^{t}+1\right)\right]^{1 / q}, \quad$ and $\quad g^{-1}(t)=$ $\left[\left(e^{t}-1\right) /\left(e^{t}+1\right)\right]^{1 / q}$. According to equation (8), a q-rung orthopair fuzzy Archimedean Einstein MM (qROFAEMM) operator is constructed as follows:

$$
\begin{aligned}
\operatorname{qROFAEMM}^{\Delta}\left(\Xi_{1}, \Xi_{2}, \ldots, \Xi_{n}\right) \\
=\left\langle\left(\frac{2\left(\mu^{\prime}-1\right)^{1 /\left(\sum_{i=1}^{n} \delta_{i}\right)}}{\left(\mu^{\prime}+3\right)^{1 /\left(\Sigma_{i=1}^{n} \delta_{i}\right)}+\left(\mu^{\prime}-1\right)^{1 /\left(\sum_{i=1}^{n} \delta_{i}\right)}}\right)^{1 / q},\right. \\
\\
\left.\qquad\left(\frac{\left(\nu^{\prime}+3\right)^{1 /\left(\sum_{i=1}^{n} \delta_{i}\right)}-\left(\nu^{\prime}-1\right)^{1 /\left(\sum_{i=1}^{n} \delta_{i}\right)}}{\left(\nu^{\prime}+3\right)^{1 /\left(\sum_{i=1}^{n} \delta_{i}\right)}+\left(\nu^{\prime}-1\right)^{1 /\left(\sum_{i=1}^{n} \delta_{i}\right)}}\right)^{1 / q}\right\rangle,
\end{aligned}
$$

where

$$
\begin{aligned}
& \mu^{\prime}=\left(\prod_{p \in \mathbf{P}_{n}}\left(\frac{\prod_{i=1}^{n}\left(\left(2-\mu_{p(i)}^{q}\right) / \mu_{p(i)}^{q}\right)^{\delta_{i}}+3}{\prod_{i=1}^{n}\left(\left(2-\mu_{p(i)}^{q}\right) / \mu_{p(i)}^{q}\right)^{\delta_{i}}-1}\right)\right)^{1 / n !}, \\
& \nu^{\prime}=\left(\prod_{p \in \mathbf{P}_{n}}\left(\frac{\prod_{i=1}^{n}\left(\left(1+\nu_{p(i)}^{q}\right) /\left(1-v_{p(i)}^{q}\right)\right)^{\delta_{i}}+3}{\prod_{i=1}^{n}\left(\left(1+v_{p(i)}^{q}\right) /\left(1-v_{p(i)}^{q}\right)\right)^{\delta_{i}}-1}\right)\right)^{1 / n !} .
\end{aligned}
$$


(8) If $f(t)=\operatorname{In}\left\{\left[\lambda+(1-\lambda) t^{q}\right] / t^{q}\right\} \quad(\lambda>0)$, then $g(t)=$ $\operatorname{In}\left\{\left[\lambda+(1-\lambda)\left(1-t^{q}\right)\right] /\left(1-t^{q}\right)\right\}, \quad f^{-1}(t)=\left[\lambda /\left(e^{t}+\right.\right.$ $\lambda-1)]^{1 / q}$, and $g^{-1}(t)=\left[\left(\mathrm{e}^{t}-1\right) /\left(\mathrm{e}^{t}+\lambda-1\right)\right]^{1 / q}$. According to equation (8), a q-rung orthopair fuzzy Archimedean Hamacher MM (qROFAHMM) operator is constructed as follows:

$\operatorname{qROFAHMM}^{\Delta}\left(\Xi_{1}, \Xi_{2}, \ldots, \Xi_{n}\right)$

$$
\begin{aligned}
= & \left\langle\left(\frac{\lambda\left(\mu^{\prime}-1\right)^{1 /\left(\sum_{i=1}^{n} \delta_{i}\right)}}{\left(\mu^{\prime}+\lambda^{2}-1\right)^{1 /\left(\sum_{i=1}^{n} \delta_{i}\right)}+(\lambda-1)\left(\mu^{\prime}-1\right)^{1 /\left(\sum_{i=1}^{n} \delta_{i}\right)}}\right)^{1 / q},\right. \\
& \left.\left(\frac{\left(\nu^{\prime}+\lambda^{2}-1\right)^{1 /\left(\sum_{i=1}^{n} \delta_{i}\right)}-\left(v^{\prime}-1\right)^{1 /\left(\sum_{i=1}^{n} \delta_{i}\right)}}{\left(\nu^{\prime}+\lambda^{2}-1\right)^{1 /\left(\sum_{i=1}^{n} \delta_{i}\right)}+(\lambda-1)\left(\nu^{\prime}-1\right)^{1 /\left(\sum_{i=1}^{n} \delta_{i}\right)}}\right)^{1 / q}\right\rangle,
\end{aligned}
$$

where

$$
\begin{aligned}
& \mu^{\prime}=\left(\prod_{p \in \mathbf{P}_{n}}\left(\frac{\prod_{i=1}^{n}\left(\left(\lambda+(1-\lambda) \mu_{p(i)}^{q}\right) / \mu_{p(i)}^{q}\right)^{\delta_{i}}+\lambda^{2}-1}{\prod_{i=1}^{n}\left(\left(\lambda+(1-\lambda) \mu_{p(i)}^{q}\right) / \mu_{p(i)}^{q}\right)^{\delta_{i}}-1}\right)\right)^{1 / n !}, \\
& \nu^{\prime}=\left(\prod_{p \in \mathbf{P}_{n}}\left(\frac{\prod_{i=1}^{n}\left(\left(\lambda+(1-\lambda)\left(1-v_{p(i)}^{q}\right)\right) /\left(1-v_{p(i)}^{q}\right)\right)^{\delta_{i}}+\lambda^{2}-1}{\prod_{i=1}^{n}\left(\left(\lambda+(1-\lambda)\left(1-\gamma_{p(i)}^{q}\right)\right) /\left(1-v_{p(i)}^{q}\right)\right)^{\delta_{i}}-1}\right)\right)^{1 / n !} .
\end{aligned}
$$

(9) If $f(t)=-\operatorname{In}\left[(\varepsilon-1) /\left(\varepsilon^{y}-1\right)\right]\left(y=t^{q} ; \varepsilon>1\right)$, then $g(t)=-\operatorname{In}\left[(\varepsilon-1) /\left(\varepsilon^{1-y}-1\right)\right], \quad f^{-1}(t)=\left\{\log _{\varepsilon}[(\varepsilon-\right.$ $\left.\left.\left.1+e^{-t}\right) / e^{-t}\right]\right\}^{1 / q}$, and $g^{-1}(t)=\left\{1-\log _{\varepsilon}\left[\left(\varepsilon-1+e^{-t}\right)\right.\right.$ $\left.\left.\mid e^{-t}\right]\right\}^{1 / q}$. According to equation (8), a q-rung orthopair fuzzy Archimedean Frank MM (qROFAFMM) operator is constructed as follows:

$$
\begin{aligned}
& \operatorname{qROFAFMM}^{\Delta}\left(\Xi_{1}, \Xi_{2}, \ldots, \Xi_{n}\right) \\
& =\left\langle\left(\log \left(1+\frac{\left(\varepsilon^{\mu^{\prime \prime}}-1\right)^{1 /\left(\sum_{i=1}^{n} \delta_{i}\right)}}{(\varepsilon-1)^{1 /\left(\sum_{i=1}^{n} \delta_{i}\right)-1}}\right)\right)^{1 / q},\right. \\
& \\
& \left.\quad\left(1-\log _{\varepsilon}\left(1+\frac{\left(\varepsilon^{1-\nu^{\prime \prime}}-1\right)^{1 /\left(\sum_{i=1}^{n} \delta_{i}\right)}}{(\varepsilon-1)^{1 /\left(\sum_{i=1}^{n} \delta_{i}\right)-1}}\right)\right)^{1 / q}\right\rangle,
\end{aligned}
$$

where

$$
\begin{aligned}
& \mu^{\prime \prime}=1-\log _{\varepsilon} \frac{\varepsilon-1+\left(\prod_{p \in \mathbf{P}_{n}}\left((\varepsilon-1) /\left(\varepsilon^{1-\mu^{\prime}}-1\right)\right)\right)^{1 / n !}}{\left(\prod_{p \in \mathbf{P}_{n}}\left((\varepsilon-1) /\left(\varepsilon^{1-\mu^{\prime}}-1\right)\right)\right)^{1 / n !}}, \\
& v^{\prime \prime}=\log _{\varepsilon} \frac{\varepsilon-1+\left(\prod_{p \in \mathbf{P}_{n}}\left((\varepsilon-1) /\left(\varepsilon^{v^{\prime}}-1\right)\right)\right)^{1 / n !}}{\left(\prod_{p \in \mathbf{P}_{n}}\left((\varepsilon-1) /\left(\varepsilon^{v^{\prime}}-1\right)\right)\right)^{1 / n !}}, \\
& \mu^{\prime}=\log _{\varepsilon} \frac{\varepsilon-1+\prod_{i=1}^{n}\left((\varepsilon-1) /\left(\varepsilon^{\mu_{p(i)}^{q}}-1\right)\right)^{\delta_{i}}}{\prod_{i=1}^{n}\left((\varepsilon-1) /\left(\varepsilon^{\mu_{p(i)}^{q}}-1\right)\right)^{\delta_{i}}}, \\
& \nu^{\prime}=1-\log _{\varepsilon} \frac{\varepsilon-1+\prod_{i=1}^{n}\left((\varepsilon-1) /\left(\varepsilon^{1-v_{p(i)}^{q}}-1\right)\right)^{\delta_{i}}}{\prod_{i=1}^{n}\left((\varepsilon-1) /\left(\varepsilon^{1-v_{p(i)}^{q}}-1\right)\right)^{\delta_{i}}} .
\end{aligned}
$$

3.1.2. qROFWAMM Operator. The qROFAMM operator has advantages in having desirable generality and flexibility and capturing the interrelationships among multiple aggregated qROFNs. But it does not consider the relative importance of each aggregated qROFN. To make up for this deficiency, weights are introduced and a qROFWAMM operator is presented. The formal definition of the presented operator is as follows.

Definition 9. Let $\left(\Xi_{1}, \Xi_{2}, \ldots, \Xi_{n}\right)\left(\Xi_{i}=<\mu_{i}, v_{i}>, i=1,2, \ldots, n\right)$ be a collection of $n$ qROFNs $(q=1,2,3, \ldots), \Delta=\left(\delta_{1}, \delta_{2}, \ldots\right.$, $\left.\delta_{n}\right)\left(\delta_{1}, \delta_{2}, \ldots, \delta_{n} \geq 0\right.$ but not at the same time $\delta_{1}=\delta_{2}=\ldots=\delta_{n}=0$ ) be a collection of $n$ real numbers, $p(i)$ be a permutation of $(1,2, \ldots, n), \mathbf{P}_{n}$ be the set of all permutations of $(1,2, \ldots, n), \Xi_{i} \oplus \Xi_{j}$ and $\Xi_{i} \otimes \Xi_{j}(i, j=1,2, \ldots, n)$ be, respectively, the sum and product operations of $\Xi_{i}$ and $\Xi_{j}$ based on ATT, $\sigma \Xi_{r}$ and $\Xi_{s}^{\tau}(r, s=1,2, \ldots, n ; \sigma, \tau>0)$ be, respectively, the multiplication operation of $\Xi_{r}$ and the power operation of $\Xi_{s}$ based on ATT, and $w_{1}, w_{2}, \ldots, w_{n}$ be, respectively, the weights of $\Xi_{1}, \Xi_{2}, \ldots, \Xi_{n}$ such that $0 \leq w_{1}$, $w_{2}, \ldots, w_{n} \leq 1$ and $w_{1}+w_{2}+\ldots+w_{n}=1$. Then, the aggregation function

$$
\begin{aligned}
& \operatorname{qROFWAMM}^{\Delta}\left(\Xi_{1}, \Xi_{2}, \ldots, \Xi_{n}\right) \\
& =\left(\frac{1}{n !} \underset{p \in \mathbf{P}_{n}}{\oplus} \otimes_{i=1}^{n}\left(\left(n w_{p(i)}\right) \Xi_{p(i)}\right)^{\delta_{i}}\right)^{1 / \Sigma_{i=1}^{n} \delta_{i},}
\end{aligned}
$$

is called the qROFWAMM operator.

According to equations (1)-(4) and (22), the following theorem is obtained.

Theorem 5. Let $\left(\Xi_{1}, \Xi_{2}, \ldots, \Xi_{n}\right)\left(\Xi_{i}=\left\langle\mu_{i}, v_{i}>, i=1,2, \ldots, n\right)\right.$ be a collection of $n$ qROFNs $(q=1,2,3, \ldots)$. Then 


$$
\begin{aligned}
& \operatorname{qROFWAmM~}^{\Delta}\left(\Xi_{1}, \Xi_{2}, \ldots, \Xi_{n}\right) \\
& =\left\langle f^{-1}\left(\frac{1}{\sum_{i=1}^{n} \delta_{i}} f\left(g^{-1}\left(\frac{1}{n !} \sum_{p \in \mathbf{P}_{n}} g\left(f^{-1}\left(\sum_{i=1}^{n}\left(\delta_{i} f\left(g^{-1}\left(\left(n w_{p(i)}\right) g\left(\mu_{p(i)}\right)\right)\right)\right)\right)\right)\right)\right)\right),\right. \\
& \left.g^{-1}\left(\frac{1}{\sum_{i=1}^{n} \delta_{i}} g\left(f^{-1}\left(\frac{1}{n !} \sum_{p \in \mathbf{P}_{n}} f\left(g^{-1}\left(\sum_{i=1}^{n}\left(\delta_{i} g\left(f^{-1}\left(\left(n w_{p(i)}\right) f\left(v_{p(i)}\right)\right)\right)\right)\right)\right)\right)\right)\right)\right\rangle,
\end{aligned}
$$

and $q \operatorname{ROFWAMM} M^{\Delta}\left(\Xi_{1}, \Xi_{2}, \ldots, \Xi_{n}\right)$ is still a $q R O F N$.

For the details of the proof of this theorem, please refer to Appendix E. In addition, it is similar to prove that the qROFWAMM operator has the properties of monotonicity and boundedness (Please note that the qROFWAMM operator no longer has idempotency).

Like equation (8), equation (23) is a generalized form of the qROFWAMM operator. If specific values are assigned to $q$ and $\delta_{1}, \delta_{2}, \ldots, \delta_{n}$ and specific forms are assigned to $f$, then specific operators can be obtained:

(1) If $q=1$, then the qROFWAMM operator will reduce to an intuitionistic fuzzy weighted Archimedean MM operator.

(2) If $q=2$, then the qROFWAMM operator will reduce to a Pythagorean fuzzy weighted Archimedean MM operator.

(3) If $\delta_{1}=\delta>0$ and $\delta_{2}=\delta_{3}=\ldots=\delta_{n}=0$, then the qROFWAMM operator will reduce to a q-rung orthopair fuzzy weighted Archimedean generalized arithmetic averaging operator.

(4) If $\delta_{1}, \delta_{2}>0$ and $\delta_{3}=\delta_{4}=\ldots=\delta_{n}=0$, then the qROFAMM operator will reduce to the q-rung orthopair fuzzy weighted Archimedean BM operator presented by Liu and Wang [42].

(5) If $\delta_{1}=\delta_{2}=\ldots=\delta_{k}=1$ and $\delta_{k+1}=\delta_{k+2}=\ldots=\delta_{n}=0$, then the qROFWAMM operator will reduce to a q-rung orthopair fuzzy weighted Archimedean MSM operator.

(6) If $\delta_{1}=\delta_{2}=\ldots=\delta_{n}=\delta>0$, then the qROFWAMM operator will reduce to a q-rung orthopair fuzzy weighted Archimedean generalized geometric averaging operator.

(7) If $f(t)=-\operatorname{In} t^{q}$, then $g(t)=-\operatorname{In}\left(1-t^{q}\right), f^{-1}(t)=\left(e^{-t}\right)^{1 / q}$, and $g^{-1}(t)=\left(1-e^{-t}\right)^{1 / q}$. According to equation (23), a q-rung orthopair fuzzy weighted Archimedean Algebraic MM (qROFWAAMM) operator is constructed as follows:

$$
\begin{aligned}
\operatorname{qROFWAAMM}^{\Delta}\left(\Xi_{1}, \Xi_{2}, \ldots, \Xi_{n}\right)= & \left\langle\left(1-\left(\prod_{p \in \mathbf{P}_{n}}\left(1-\prod_{i=1}^{n}\left(1-\left(1-\mu_{p(i)}^{q}\right)^{n w_{p(i)}}\right)^{\delta_{i}}\right)\right)^{1 / n !}\right)^{1 / \Sigma_{i=1}^{n} \delta_{i}}\right)^{1 / q}, \\
& \left.\left(1-\left(1-\left(\prod_{p \in \mathbf{P}_{n}}\left(1-\prod_{i=1}^{n}\left(1-v_{p(i)}^{q n w_{p(i)}}\right)^{\delta_{i}}\right)\right)^{1 / n !}\right)^{1 / \Sigma_{i=1}^{n} \delta_{i}}\right)^{1 / q}\right\rangle .
\end{aligned}
$$

This operator is actually the q-rung orthopair fuzzy weighted MM operator presented by Wang et al. [50]. It has the following special cases:
(1) If $q=1$, then the qROFWAAMM operator will reduce to

$$
\left\langle\left(1-\left(\prod_{p \in \mathbf{P}_{n}}\left(1-\prod_{i=1}^{n}\left(1-\left(1-\mu_{p(i)}\right)^{n w_{p(i)}}\right)^{\delta_{i}}\right)\right)^{1 / n !}\right)^{1 / \Sigma_{i=1}^{n} \delta_{i}}, 1-\left(1-\left(\prod_{p \in \mathbf{P}_{n}}\left(1-\prod_{i=1}^{n}\left(1-\nu_{p(i)}^{n w_{p(i)}}\right)^{\delta_{i}}\right)^{1 / n !}\right)^{1 / \Sigma_{i=1}^{n} \delta_{i}}\right\rangle\right.
$$

which is the intuitionistic fuzzy weighted MM operator presented by Liu and Li [55].
(2) If $q=2$, then the qROFWAAMM operator will reduce to 


$$
\left\langle\left(\left(1-\left(\prod_{p \in \mathbf{P}_{n}}\left(1-\prod_{i=1}^{n}\left(1-\left(1-\mu_{p(i)}^{2}\right)^{n w_{p(i)}}\right)^{\delta_{i}}\right)\right)^{1 / n !}\right)^{1 / \Sigma_{i=1}^{n} \delta_{i}}\right)^{1 / 2},\left(1-\left(1-\left(\prod_{p \in \mathbf{P}_{n}}\left(1-\prod_{i=1}^{n}\left(1-v_{p(i)}^{2 n w_{p(i)}}\right)^{\delta_{i}}\right)\right)^{1 / n !}\right)^{1 / \Sigma_{i=1}^{n} \delta_{i}}\right)^{1 / 2}\right\rangle
$$

which is the Pythagorean fuzzy weighted MM operator presented by Zhu and Li [56].
(3) If $\delta_{1}=\delta>0$ and $\delta_{2}=\delta_{3}=\ldots=\delta_{n}=0$, then the qROFWAAMM operator will reduce to

$$
\left\langle\left(\left(1-\left(\prod_{i=1}^{n}\left(1-\left(1-\left(1-\mu_{i}^{q}\right)^{n w_{i}}\right)^{\delta}\right)\right)^{1 / n}\right)^{1 / \delta}\right)^{1 / q},\left(1-\left(1-\left(\prod_{i=1}^{n}\left(1-\left(1-v_{i}^{q n w_{i}}\right)^{\delta}\right)\right)^{1 / n}\right)^{1 / \delta}\right)^{1 / q}\right\rangle,
$$

which is a q-rung orthopair fuzzy weighted generalized arithmetic averaging operator.
(4) If $\delta_{1}, \delta_{2}>0$ and $\delta_{3}=\delta_{4}=\ldots=\delta_{n}=0$, then the qROFWAAMM operator will reduce to

$$
\begin{aligned}
& \left\langle\left(\left(1-\left(\prod_{\substack{i, j=1 \\
j \neq i}}^{n}\left(1-\left(1-\left(1-\mu_{i}^{q}\right)^{n w_{i}}\right)^{\delta_{1}}\left(1-\left(1-\mu_{j}^{q}\right)^{n w_{j}}\right)^{\delta_{2}}\right)\right)^{1 / n(n-1)}\right)^{1 /\left(\delta_{1}+\delta_{2}\right)}\right)^{1 / q},\right. \\
& \left.\left(1-\left(1-\left(\prod_{\substack{i, j=1 \\
j \neq i}}^{n}\left(1-\left(1-v_{i}^{q n w_{i}}\right)^{\delta_{1}}\left(1-v_{j}^{q n w_{j}}\right)^{\delta_{2}}\right)\right)^{1 / n(n-1)}\right)^{1 /\left(\delta_{1}+\delta_{2}\right)}\right)^{1 / q}\right)
\end{aligned}
$$

which is the q-rung orthopair fuzzy weighted Archimedean Algebraic BM operator presented by Liu and Wang [42].
(5) If $\delta_{1}=\delta_{2}=\ldots=\delta_{k}=1$ and $\delta_{k+1}=\delta_{k+2}=\ldots=\delta_{n}=0$, then the GROFAAMM operator will reduce to

$$
\begin{aligned}
& \left\langle\left(\left(1-\prod_{1 \leq i_{1}<\ldots<i_{k} \leq n}^{n}\left(1-\prod_{j=1}^{k}\left(1-\left(1-\mu_{i_{j}}^{q}\right)^{n w_{i_{j}}}\right)\right)^{k !(n-k) ! n !}\right)^{1 / k}\right)^{1 / q},\right. \\
& \left.\left(1-\left(1-\prod_{1 \leq i_{1}<\ldots<i_{k} \leq n}^{n}\left(1-\prod_{j=1}^{k}\left(1-v_{i_{j}}^{q n w_{i_{j}}}\right)\right)^{k !(n-k) ! n !}\right)^{1 / k}\right)^{1 / q}\right\rangle,
\end{aligned}
$$

which is the q-rung orthopair fuzzy weighted MSM operator presented by Wei et al. [47] and Liu et al. [48].
(6) If $\delta_{1}=\delta_{2}=\ldots=\delta_{n}=\delta>0$, then the qROFWAAMM operator will reduce to 


$$
\left\langle\left(1-\left(1-\prod_{i=1}^{n}\left(1-\left(1-\mu_{i}^{q n w_{i}}\right)^{\delta}\right)^{1 / n}\right)^{1 / \delta}\right)^{1 / q},\left(\left(1-\prod_{i=1}^{n}\left(1-\left(1-\left(1-v_{i}^{q}\right)^{n w_{i}}\right)^{\delta}\right)^{1 / n}\right)^{1 / \delta}\right)^{1 / q}\right\rangle
$$

which is a q-rung orthopair fuzzy weighted generalized geometric averaging operator.

(7) If $f(t)=\operatorname{In}\left[\left(2-t^{q}\right) / t^{q}\right]$, then $g(t)=\operatorname{In}\left[\left(1+t^{q}\right) /\left(1-t^{q}\right)\right]$, $f^{-1}(t)=\left[2 /\left(\mathrm{e}^{t}+1\right)\right]^{1 / q}$, and $g^{-1}(t)=\left[\left(\mathrm{e}^{t}-1\right) /\left(\mathrm{e}^{t}+1\right)\right]^{1 / q}$.
According to equation (23), a q-rung orthopair fuzzy weighted Archimedean Einstein MM (qROFWAEMM) operator is constructed as follows:

$\operatorname{qROFWAEMM}^{\Delta}\left(\Xi_{1}, \Xi_{2}, \ldots, \Xi_{n}\right)=\left\langle\left(\frac{2\left(\mu^{\prime}-1\right)^{1 /\left(\sum_{i=1}^{n} \delta_{i}\right)}}{\left(\mu^{\prime}+3\right)^{1 /\left(\Sigma_{i=1}^{n} \delta_{i}\right)}+\left(\mu^{\prime}-1\right)^{1 /\left(\sum_{i=1}^{n} \delta_{i}\right)}}\right)^{1 / q},\left(\frac{\left(\nu^{\prime}+3\right)^{1 /\left(\sum_{i=1}^{n} \delta_{i}\right)}-\left(\nu^{\prime}-1\right)^{1 /\left(\sum_{i=1}^{n} \delta_{i}\right)}}{\left(\nu^{\prime}+3\right)^{1 /\left(\sum_{i=1}^{n} \delta_{i}\right)}+\left(\nu^{\prime}-1\right)^{1 /\left(\sum_{i=1}^{n} \delta_{i}\right)}}\right)^{1 / q}\right\rangle$,

where

$$
\begin{aligned}
& \mu^{\prime}=\left(\prod_{p \in \mathbf{P}_{n}}\left(\frac{\prod_{i=1}^{n}\left(\left(\left(1+\mu_{p(i)}^{q}\right)^{n w_{p(i)}}+3\left(1-\mu_{p(i)}^{q}\right)^{n w_{p(i)}}\right) /\left(\left(1+\mu_{p(i)}^{q}\right)^{n w_{p(i)}}-\left(1-\mu_{p(i)}^{q}\right)^{n w_{p(i)}}\right)\right)^{\delta_{i}}+3}{\prod_{i=1}^{n}\left(\left(\left(1+\mu_{p(i)}^{q}\right)^{n w_{p(i)}}+3\left(1-\mu_{p(i)}^{q}\right)^{n w_{p(i)}}\right) /\left(\left(1+\mu_{p(i)}^{q}\right)^{n w_{p(i)}}-\left(1-\mu_{p(i)}^{q}\right)^{n w_{p(i)}}\right)\right)^{\delta_{i}}-1}\right)^{1 / n !},\right. \\
& \nu^{\prime}=\left(\prod_{p \in \mathbf{P}_{n}}\left(\frac{\prod_{i=1}^{n}\left(\left(\left(2-v_{p(i)}^{q}\right)^{n w_{p(i)}}+3 v_{p(i)}^{q n w_{p(i)}}\right) /\left(\left(2-v_{p(i)}^{q}\right)^{n w_{p(i)}}-v_{p(i)}^{q n w_{p(i)}}\right)\right)^{\delta_{i}}+3}{\prod_{i=1}^{n}\left(\left(\left(2-v_{p(i)}^{q}\right)^{n w_{p(i)}}+3 v_{p(i)}^{q n w_{p(i)}}\right) /\left(\left(2-v_{p(i)}^{q}\right)^{p(i)}-v_{p(i)}^{q n w_{p(i)}}\right)\right)^{\delta_{i}}-1}\right)\right)^{1 / n !} .
\end{aligned}
$$

(8) If $f(t)=\operatorname{In}\left\{\left[\lambda+(1-\lambda) t^{q}\right] / t^{q}\right\} \quad(\lambda>0)$, then $g(t)=\operatorname{In}$ $\left\{\left[\lambda+(1-\lambda)\left(1-t^{q}\right)\right] /\left(1-t^{q}\right)\right\}, f^{-1}(t)=\left[\lambda /\left(e^{t}+\lambda-1\right)\right]^{1 / q}$, and $g^{-1}(t)=\left[\left(e^{t}-1\right) /\left(e^{t}+\lambda-1\right)\right]^{1 / q}$. According to equation (23), a q-rung orthopair fuzzy weighted Archimedean Hamacher MM (qROFWAHMM) operator is constructed as follows:

$$
\begin{aligned}
\operatorname{qROFWAHMM}^{\Delta}\left(\Xi_{1}, \Xi_{2}, \ldots, \Xi_{n}\right)= & \left\langle\left(\frac{\lambda\left(\mu^{\prime}-1\right)^{1 /\left(\Sigma_{i=1}^{n} \delta_{i}\right)}}{\left(\mu^{\prime}+\lambda^{2}-1\right)^{1 /\left(\sum_{i=1}^{n} \delta_{i}\right)}+(\lambda-1)\left(\mu^{\prime}-1\right)^{1 /\left(\sum_{i=1}^{n} \delta_{i}\right)}}\right)^{1 / q},\right. \\
& \left.\left(\frac{\left(\nu^{\prime}+\lambda^{2}-1\right)^{1 /\left(\sum_{i=1}^{n} \delta_{i}\right)}-\left(\nu^{\prime}-1\right)^{1 /\left(\sum_{i=1}^{n} \delta_{i}\right)}}{\left(\nu^{\prime}+\lambda^{2}-1\right)^{1 /\left(\sum_{i=1}^{n} \delta_{i}\right)}+(\lambda-1)\left(\nu^{\prime}-1\right)^{1 /\left(\sum_{i=1}^{n} \delta_{i}\right)}}\right)^{1 / q}\right\rangle,
\end{aligned}
$$

where 


$$
\begin{aligned}
& \mu^{\prime}=\left(\prod_{p \in \mathbb{P}_{n}}\left(\frac{\prod_{i=1}^{n}\left(\left(\lambda+(1-\lambda)\left(1-\mu_{p(i)}^{q}\right)\right)^{n w_{p(i)}}+\left(\lambda^{2}-1\right)\left(1-\mu_{p(i)}^{q}\right)^{n w_{p(i)}}\right) /\left(\left(\lambda+(1-\lambda)\left(1-\mu_{p(i)}^{q}\right)\right)^{n w_{p(i)}}-\left(1-\mu_{p(i)}^{q}\right)^{n w_{p(i)}}\right)^{\delta_{i}}+\lambda^{2}-1}{\prod_{i=1}^{n}\left(\left(\left(\lambda+(1-\lambda)\left(1-\mu_{p(i)}^{q}\right)\right)^{n w_{p(i)}}+\left(\lambda^{2}-1\right)\left(1-\mu_{p(i)}^{q}\right)^{n w_{p(i)}}\right) /\left(\lambda+(1-\lambda)\left(1-\mu_{p(i)}^{q}\right)\right)^{n w_{p(i)}}-\left(1-\mu_{p(i)}^{q}\right)^{n w_{p(i)}}\right)^{\delta_{i}}-1}\right)\right)^{1 / n !}, \\
& \nu^{\prime}=\left(\prod_{p \in \mathbf{P}_{n}}\left(\frac{\prod_{i=1}^{n}\left(\left(\left(\lambda+(1-\lambda) v_{p(i)}^{q}\right)^{n w_{p(i)}}+\left(\lambda^{2}-1\right) v_{p(i)}^{q n w_{(i)}}\right) /\left(\left(\lambda+(1-\lambda) v_{p(i)}^{q}\right)^{n w_{p(i)}}-v_{p(i)}^{q n w_{p(i)}}\right)\right)^{\delta_{i}}+\lambda^{2}-1}{\prod_{i=1}^{n}\left(\left(\left(\lambda+(1-\lambda) v_{p(i)}^{q}\right)^{n w_{p(i)}}+\left(\lambda^{2}-1\right) v_{p(i)}^{q n \omega_{p(i)}}\right) /\left(\left(\lambda+(1-\lambda) v_{p(i)}^{q}\right)^{n w_{p(i)}}-v_{p(i)}^{q n w_{p(i)}}\right)\right)^{\delta_{i}}-1}\right)\right)^{1 / n !} .
\end{aligned}
$$

(9) If $f(t)=-\operatorname{In}\left[(\varepsilon-1) /\left(\varepsilon^{y}-1\right)\right] \quad\left(y=t^{q} ; \quad \varepsilon>1\right)$, then $g(t)=-\operatorname{In}\left[(\varepsilon-1) /\left(\varepsilon^{1-y}-1\right)\right], f^{-1}(t)=\left\{\log _{\varepsilon}\left[\left(\varepsilon-1+e^{-t}\right) /\right.\right.$ $\left.\left.e^{-t}\right]\right\}^{1 / q}, \quad$ and $\quad g^{-1}(t)=\left\{1-\log _{\varepsilon}\left[\left(\varepsilon-1+e^{-t}\right) / e^{-t}\right]\right\}^{1 / q}$.
According to equation (23), a q-rung orthopair fuzzy weighted Archimedean Frank MM (qROFWAFMM) operator is constructed as follows:

$$
\begin{aligned}
& \operatorname{qROFWAFMM}^{\Delta}\left(\Xi_{1}, \Xi_{2}, \ldots, \Xi_{n}\right) \\
& =\left\langle\left(\log _{\varepsilon}\left(1+\frac{(\varepsilon-1)\left(\varepsilon^{\mu^{\prime \prime \prime}}-1\right)^{1 /\left(\sum_{i=1}^{n} \delta_{i}\right)}}{(\varepsilon-1)^{1 /\left(\sum_{i=1}^{n} \delta_{i}\right)}}\right)\right)^{1 / q},\left(1-\log _{\varepsilon}\left(1+\frac{(\varepsilon-1)\left(\varepsilon^{1-\nu^{\prime \prime \prime}}-1\right)^{1 /\left(\sum_{i=1}^{n} \delta_{i}\right)}}{(\varepsilon-1)^{1 /\left(\sum_{i=1}^{n} \delta_{i}\right)}}\right)\right)^{1 / q}\right\rangle,
\end{aligned}
$$

where

$$
\begin{aligned}
& \mu^{\prime \prime \prime}=1-\log _{\varepsilon} \frac{\varepsilon-1+\left(\prod_{p \in \mathbf{P}_{n}}\left((\varepsilon-1) /\left(\varepsilon^{1-\mu^{\prime \prime}}-1\right)\right)\right)^{1 / n !}}{\left(\prod_{p \in \mathbf{P}_{n}}\left((\varepsilon-1) /\left(\varepsilon^{1-\mu^{\prime \prime}}-1\right)\right)\right)^{1 / n !}}, \\
& \nu^{\prime \prime \prime}=\log _{\varepsilon} \frac{\varepsilon-1+\left(\prod_{p \in \mathbf{P}_{n}}\left((\varepsilon-1) /\left(\varepsilon^{\nu^{\prime \prime}}-1\right)\right)\right)^{1 / n !}}{\left(\prod_{p \in \mathbf{P}_{n}}\left((\varepsilon-1) /\left(\varepsilon^{\nu^{\prime \prime}}-1\right)\right)\right)^{1 / n !}}, \\
& \mu^{\prime \prime}=\log _{\varepsilon} \frac{\varepsilon-1+\prod_{i=1}^{n}\left((\varepsilon-1) /\left(\varepsilon^{u^{\prime}}-1\right)\right)^{\delta_{i}}}{\prod_{i=1}^{n}\left((\varepsilon-1) /\left(\varepsilon^{u^{\prime}}-1\right)\right)^{\delta_{i}}}, \\
& v^{\prime \prime}=1-\log _{\varepsilon} \frac{\varepsilon-1+\prod_{i=1}^{n}\left((\varepsilon-1) /\left(\varepsilon^{1-\gamma^{\prime}}-1\right)\right)^{\delta_{i}}}{\prod_{i=1}^{n}\left((\varepsilon-1) /\left(\varepsilon^{1-\nu^{\prime}}-1\right)\right)^{\delta_{i}}}, \\
& \mu^{\prime}=1-\log _{\varepsilon}\left(1+\frac{\left(\varepsilon^{1-\mu_{p(i)}^{q}}-1\right)^{n w_{p(i)}}}{(\varepsilon-1)^{n w_{p(i)}-1}}\right), \\
& v^{\prime}=\log _{\varepsilon}\left(1+\frac{\left(\varepsilon^{\left.v_{p(i)}^{q}-1\right)^{n w_{p(i)}}}\right)}{(\varepsilon-1)^{n w_{p(i)}-1}}\right) .
\end{aligned}
$$

\subsection{Archimedean GMM Operators}

3.2.1. qROFAGMM Operator. A qROFAGMM operator is a GMM operator for aggregating qROFNs, in which the sum, product, multiplication, and power operations are performed using the operational rules of qROFNs based on ATT. Its formal definition is as follows.

Definition 10. Let $\left(\Xi_{1}, \Xi_{2}, \ldots, \Xi_{n}\right)\left(\Xi_{i}=<\mu_{i}, v_{i}>, i=1,2, \ldots\right.$, $n)$ be a collection of $n$ qROFNs $(q=1,2,3, \ldots), \Delta=\left(\delta_{1}, \delta_{2}\right.$, $\left.\ldots, \delta_{n}\right)\left(\delta_{1}, \delta_{2}, \ldots, \delta_{n} \geq 0\right.$ but not at the same time $\delta_{1}=\delta_{2}=\ldots=\delta_{n}=0$ ) be a collection of $n$ real numbers, $p(i)$ be a permutation of $(1,2, \ldots, n), \mathbf{P}_{n}$ be the set of all permutations of $(1,2, \ldots, n), \Xi_{i} \oplus \Xi_{j}$ and $\Xi_{i} \otimes \Xi_{j}(i, j=1,2, \ldots, n)$ be, respectively, the sum and product operations of $\Xi_{i}$ and $\Xi_{j}$ based on ATT, and $\sigma \Xi_{r}$ and $\Xi_{s}^{\tau}(r, s=1,2, \ldots, n ; \sigma, \tau>0)$ be, respectively, the multiplication operation of $\Xi_{r}$ and the power operation of $\Xi_{s}$ based on ATT. Then the aggregation function

$$
\operatorname{qROFAGMM}^{\Delta}\left(\Xi_{1}, \Xi_{2}, \ldots, \Xi_{n}\right)=\frac{1}{\sum_{i=1}^{n} \delta_{i}}\left(\underset{p \in \mathbb{P}_{n}}{\otimes} \underset{i=1}{\stackrel{n}{\oplus}}\left(\delta_{i} \Xi_{p(i)}\right)\right)^{1 / n !},
$$

is called the qROFAGMM operator.

According to equations (1)-(4) and (37), the following theorem is obtained.

Theorem 6. Let $\left(\Xi_{1}, \Xi_{2}, \ldots, \Xi_{n}\right)\left(\Xi_{i}=\left\langle\mu_{i}, v_{i}>, i=1,2, \ldots, n\right)\right.$ be a collection of $n$ qROFNs $(q=1,2,3, \ldots)$. Then 


$$
\begin{aligned}
\operatorname{qROFAGMM}^{\Delta}\left(\Xi_{1}, \Xi_{2}, \ldots, \Xi_{n}\right)= & \left\langleg ^ { - 1 } \left(\frac{1}{\sum_{i=1}^{n} \delta_{i}} g\left(f^{-1}\left(\frac{1}{n !} \sum_{p \in \mathbf{P}_{n}} f\left(g^{-1}\left(\sum_{i=1}^{n}\left(\delta_{i} g\left(\mu_{p(i)}\right)\right)\right)\right)\right)\right),\right.\right. \\
& f^{-1}\left(\frac{1}{\sum_{i=1}^{n} \delta_{i}} f\left(g^{-1}\left(\frac{1}{n !} \sum_{p \in \mathbf{P}_{n}} g\left(f^{-1}\left(\sum_{i=1}^{n}\left(\delta_{i} f\left(v_{p(i)}\right)\right)\right)\right)\right)\right)\right),
\end{aligned}
$$

and $q$ ROFAGMM ${ }^{\Delta}\left(\Xi_{1}, \Xi_{2}, \ldots, \Xi_{n}\right)$ is still a $q R O F N$.

For the details of the proof of this theorem, please refer to Appendix F. The following three theorems, respectively, state the idempotency, monotonicity, and boundedness of the qROFAGMM operator.

Theorem 7 (idempotency). Let $\left(\Xi_{1}, \Xi_{2}, \ldots, \Xi_{n}\right)\left(\Xi_{i}=\left\langle\mu_{i}, v_{i}\right\rangle\right.$, $i=1,2, \ldots, n)$ be a collection of $n q \operatorname{ROFNs}(q=1,2,3, \ldots)$. If $\Xi_{i}=\Xi=\left\langle\mu\right.$, v> for all $i=1,2, \ldots, n$, then $q \operatorname{ROFAGM} M^{\Delta}\left(\Xi_{1}\right.$, $\left.\Xi_{2}, \ldots, \Xi_{n}\right)=\Xi$.

Theorem 8 (monotonicity). Let $\left(\Xi_{1,1}, \Xi_{1,2}, \ldots, \Xi_{1, n}\right)$ $\left(\Xi_{1, i}=\left\langle\mu_{1, i}, v_{1, i}\right\rangle, i=1,2, \ldots, n\right)$ and $\left(\Xi_{2,1}, \Xi_{2,2}, \ldots, \Xi_{2, n}\right)$ $\left(\Xi_{2, i}=\left\langle\mu_{2, i}, \nu_{2, i}>\right)\right.$ be two collections of $n q R O F N s(q=1,2,3$, ...). If $\mu_{1, i} \geq \mu_{2, i}$ and $\nu_{1, i} \leq \nu_{2, i}$ for all $i=1,2, \ldots, n$, then $\operatorname{qROFAGMM}^{\Delta}\left(\Xi_{1,1}, \quad \Xi_{1,2}, \ldots, \Xi_{1, n}\right) \geq q \operatorname{ROFAGM} M^{\Delta}\left(\Xi_{2,1}\right.$, $\left.\Xi_{2,2}, \ldots, \Xi_{2, n}\right)$.

Theorem 9 (boundedness). Let $\left(\Xi_{1}, \Xi_{2}, \ldots, \Xi_{n}\right)\left(\Xi_{i}=<\mu_{i}\right.$, $\left.\nu_{i}>, i=1,2, \ldots, n\right)$ be a collection of $n q R O F N s(q=1,2,3$, ...), $\Xi_{U B}=<\max \left(\mu_{i}\right), \quad \min \left(\nu_{i}\right)>$, and $\Xi_{L B}=<\min \left(\mu_{i}\right)$, $\max \left(\nu_{i}\right)>$. Then $\Xi_{L B} \leq q \operatorname{ROFAGMM}{ }^{\Delta}\left(\Xi_{1}, \Xi_{2}, \ldots, \Xi_{n}\right) \leq \Xi_{U B}$.

For the details of the proofs of these three theorems, please refer to Appendixes G-I, respectively.

Equation (38) is a generalized form of the qROFAGMM operator. If specific values are assigned to $q$ and $\delta_{1}, \delta_{2}, \ldots, \delta_{n}$ and specific forms are assigned to $f$, then specific operators can be obtained:
(1) If $q=1$, then the qROFAGMM operator will reduce to an intuitionistic fuzzy Archimedean GMM operator.

(2) If $q=2$, then the qROFAGMM operator will reduce to a Pythagorean fuzzy Archimedean GMM operator.

(3) If $\delta_{1}=\delta>0$ and $\delta_{2}=\delta_{3}=\ldots=\delta_{n}=0$, then the qROFAGMM operator will reduce to a q-rung orthopair fuzzy Archimedean generalized geometric averaging operator.

(4) If $\delta_{1}, \delta_{2}>0$ and $\delta_{3}=\delta_{4}=\ldots=\delta_{n}=0$, then the qROFAGMM operator will reduce to a q-rung orthopair fuzzy Archimedean GBM operator.

(5) If $\delta_{1}=\delta_{2}=\ldots=\delta_{k}=1$ and $\delta_{k+1}=\delta_{k+2}=\ldots=\delta_{n}=0$, then the qROFAGMM operator will reduce to a q-rung orthopair fuzzy Archimedean GMSM operator.

(6) If $\delta_{1}=\delta_{2}=\ldots=\delta_{n}=\delta>0$, then the qROFAGMM operator will reduce to a q-rung orthopair fuzzy Archimedean generalized arithmetic averaging operator.

(7) If $f(t)=-\operatorname{In} t^{q}$, then $g(t)=-\operatorname{In}\left(1-t^{q}\right), f^{-1}(t)=\left(e^{-t}\right)^{1 / q}$, and $g^{-1}(t)=\left(1-e^{-t}\right)^{1 / q}$. According to equation (38), a q-rung orthopair fuzzy Archimedean Algebraic GMM (qROFAAGMM) operator is constructed as follows:

$$
\begin{aligned}
\operatorname{qROFAAGMM}^{\Delta}\left(\Xi_{1}, \Xi_{2}, \ldots, \Xi_{n}\right)= & \left\langle\left(1-\left(1-\left(\prod_{p \in \mathbf{P}_{n}}\left(1-\prod_{i=1}^{n}\left(1-\mu_{p(i)}^{q}\right)^{\delta_{i}}\right)\right)^{1 / n !}\right)^{1 / \Sigma_{i=1}^{n} \delta_{i}}\right)^{1 / q},\right. \\
& \left.\left.\left(\left(1-\left(\prod_{p \in \mathbf{P}_{n}}\left(1-\prod_{i=1}^{n} \nu_{p(i)}^{q \delta_{i}}\right)\right)^{1 / n !}\right)^{1 / \Sigma_{i=1}^{n} \delta_{i}}\right)^{1 / q}\right)^{1 / q}\right)
\end{aligned}
$$

This operator is actually the q-rung orthopair fuzzy GMM operator presented by Wang et al. [50]. It has the following special cases:

(1) If $q=1$, then the qROFAAGMM operator will reduce to

$$
\begin{aligned}
\left\langle 1-\left(1-\left(\prod_{p \in \mathbf{P}_{n}}\left(1-\prod_{i=1}^{n}\left(1-\mu_{p(i)}\right)^{\delta_{i}}\right)\right)^{1 / n !}\right)^{1 / \Sigma_{i=1}^{n} \delta_{i}},\right. \\
\left.\left(1-\left(\prod_{p \in \mathbf{P}_{n}}\left(1-\prod_{i=1}^{n} \nu_{p(i)}^{\delta_{i}}\right)\right)^{1 / n !}\right)^{1 / \Sigma_{i=1}^{n} \delta_{i}}\right\rangle,
\end{aligned}
$$


which is the intuitionistic fuzzy GMM operator presented by Liu and Li [55].
(2) If $q=2$, then the qROFAAGMM operator will reduce to

$$
\left\langle\left(1-\left(1-\left(\prod_{p \in \mathbf{P}_{\mathbf{n}}}\left(1-\prod_{i=1}^{n}\left(1-\mu_{p(i)}^{2}\right)^{\delta_{i}}\right)\right)^{1 / n !}\right)^{1 / \Sigma_{i=1}^{n} \delta_{i}}\right)^{1 / 2},\left(\left(1-\left(\prod_{p \in \mathbf{P}_{n}}\left(1-\prod_{i=1}^{n} v_{p(i)}^{2 \delta_{i}}\right)\right)^{1 / n !}\right)^{1 / \Sigma_{i=1}^{n} \delta_{i}}\right)^{1 / 2}\right\rangle
$$

which is the Pythagorean fuzzy GMM operator presented by Zhu and Li [56].

(3) If $\delta_{1}=\delta>0$ and $\delta_{2}=\delta_{3}=\ldots=\delta_{n}=0$, then the qROFAAGMM operator will reduce to the operator in equation (15).
(4) If $\delta_{1}, \delta_{2}>0$ and $\delta_{3}=\delta_{4}=\ldots=\delta_{n}=0$, then the qROFAAGMM operator will reduce to

$$
\left\langle\left(1-\left(1-\prod_{\substack{i, j=1 \\ j \neq i}}^{n}\left(1-\left(1-\mu_{i}^{q}\right)^{\delta_{1}}\left(1-\mu_{j}^{q}\right)^{\delta_{2}}\right)^{1 / n(n-1)}\right)^{1 /\left(\delta_{1}+\delta_{2}\right)}\right)^{1 / q},\left(\left(1-\prod_{\substack{i, j=1 \\ j \neq i}}^{n}\left(1-v_{i}^{q \delta_{1}} \gamma_{j}^{q \delta_{2}}\right)^{1 / n(n-1)}\right)^{1 /\left(\delta_{1}+\delta_{2}\right)}\right)^{1 / q}\right\rangle,
$$

which is a q-rung orthopair fuzzy Archimedean Algebraic GBM operator.
(5) If $\delta_{1}=\delta_{2}=\ldots=\delta_{k}=1$ and $\delta_{k+1}=\delta_{k+2}=\ldots=\delta_{n}=0$, then the qROFAAGMM operator will reduce to

$$
\left\langle\left(1-\left(1-\prod_{1 \leq i_{1}<\ldots<i_{k} \leq n}^{n}\left(1-\prod_{j=1}^{k}\left(1-\mu_{i_{j}}^{q}\right)\right)^{k !(n-k) ! / n !}\right)^{1 / k}\right)^{1 / q},\left(\left(1-\prod_{1 \leq i_{1}<\cdots<i_{k} \leq n}^{n}\left(1-\prod_{j=1}^{k} \nu_{i_{j}}^{q}\right)^{k !(n-k) ! / n !}\right)^{1 / k}\right)^{1 / q}\right\rangle,
$$

which is the q-rung orthopair fuzzy GMSM operator presented by Wei et al. [47].

(6) If $\delta_{1}=\delta_{2}=\ldots=\delta_{n}=\delta>0$, then the qROFAAGMM operator will reduce to the operator in equation (12).
(7) If $f(t)=\operatorname{In}\left[\left(2-t^{q}\right) / t^{q}\right]$, then $g(t)=\operatorname{In}\left[\left(1+t^{q}\right) /\left(1-t^{q}\right)\right]$, $f^{-1}(t)=\left[2 /\left(e^{t}+1\right)\right]^{1 / q}$, and $g^{-1}(t)=\left[\left(e^{t}-1\right) /\left(e^{t}+1\right)\right]^{1 / q}$. According to equation (38), a q-rung orthopair fuzzy Archimedean Einstein GMM (qROFAEGMM) operator is constructed as follows:

$$
\operatorname{qROFAEGMM}^{\Delta}\left(\Xi_{1}, \Xi_{2}, \ldots, \Xi_{n}\right)=\left\langle\left(\frac{\left(\mu^{\prime}+3\right)^{1 /\left(\sum_{i=1}^{n} \delta_{i}\right)}-\left(\mu^{\prime}-1\right)^{1 /\left(\sum_{i=1}^{n} \delta_{i}\right)}}{\left(\mu^{\prime}+3\right)^{1 /\left(\sum_{i=1}^{n} \delta_{i}\right)}+\left(\mu^{\prime}-1\right)^{1 /\left(\sum_{i=1}^{n} \delta_{i}\right)}}\right)^{1 / q},\left(\frac{2 \sum\left(\nu^{\prime}-1\right)^{1 /\left(\sum_{i=1}^{n} \delta_{i}\right)}}{\left(\nu^{\prime}+3\right)^{1 /\left(\sum_{i=1}^{n} \delta_{i}\right)}+\left(\nu^{\prime}-1\right)^{1 /\left(\sum_{i=1}^{n} \delta_{i}\right)}}\right)^{1 / q}\right\rangle,
$$


where

$$
\begin{aligned}
& \mu^{\prime}=\prod_{p \in \mathbf{P}_{n}}\left(\frac{\prod_{i=1}^{n}\left(\left(1+\mu_{p(i)}^{q}\right) /\left(1-\mu_{p(i)}^{q}\right)\right)^{\delta_{i}}+3}{\prod_{i=1}^{n}\left(\left(1+\mu_{p(i)}^{q}\right) /\left(1-\mu_{p(i)}^{q}\right)\right)^{\delta_{i}}-1}\right)^{1 / n !}, \\
& \nu^{\prime}=\prod_{p \in \mathbf{P}_{n}}\left(\frac{\prod_{i=1}^{n}\left(\left(2-\nu_{p(i)}^{q}\right) / \nu_{p(i)}^{q}\right)^{\delta_{i}}+3}{\prod_{i=1}^{n}\left(\left(2-\nu_{p(i)}^{q}\right) / \nu_{p(i)}^{q}\right)^{\delta_{i}}-1}\right)^{1 / n !} .
\end{aligned}
$$

(8) If $f(t)=\operatorname{In}\left\{\left[\lambda+(1-\lambda) t^{q}\right] / t^{q}\right\} \quad(\lambda>0)$, then $g(t)=\operatorname{In}$ $\left\{\left[\lambda+(1-\lambda)\left(1-t^{q}\right)\right] /\left(1-t^{q}\right)\right\}, f^{-1}(t)=\left[\lambda /\left(e^{t}+\lambda-1\right)\right]^{1 / q}$, and $g^{-1}(t)=\left[\left(e^{t}-1\right) /\left(e^{t}+\lambda-1\right)\right]^{1 / q}$. According to equation (38), a q-rung orthopair fuzzy Archimedean Hamacher GMM (qROFAHGMM) operator is constructed as follows:

$$
\begin{aligned}
\operatorname{qROFAHGMM}^{\Delta}\left(\Xi_{1}, \Xi_{2}, \ldots, \Xi_{n}\right)= & \left\langle\left(\frac{\left(\mu^{\prime}+\lambda^{2}-1\right)^{1 /\left(\sum_{i=1}^{n} \delta_{i}\right)}-\left(\mu^{\prime}-1\right)^{1 /\left(\sum_{i=1}^{n} \delta_{i}\right)}}{\left(\mu^{\prime}+\lambda^{2}-1\right)^{1 /\left(\sum_{i=1}^{n} \delta_{i}\right)}+(\lambda-1)\left(\mu^{\prime}-1\right)^{1 /\left(\sum_{i=1}^{n} \delta_{i}\right)}}\right)^{1 / q},\right. \\
& \left.\left(\frac{\lambda\left(\nu^{\prime}-1\right)^{1 /\left(\Sigma_{i=1}^{n} \delta_{i}\right)}}{\left(\nu^{\prime}+\lambda^{2}-1\right)^{1 /\left(\sum_{i=1}^{n} \delta_{i}\right)}+(\lambda-1)\left(\nu^{\prime}-1\right)^{1 /\left(\sum_{i=1}^{n} \delta_{i}\right)}}\right)^{1 / q}\right\rangle,
\end{aligned}
$$

where

$$
\begin{aligned}
& \mu^{\prime}=\prod_{p \in \mathbf{P}_{n}}\left(\frac{\prod_{i=1}^{n}\left(\left(\lambda+(1-\lambda)\left(1-\mu_{p(i)}^{q}\right)\right) /\left(1-\mu_{p(i)}^{q}\right)\right)^{\delta_{i}}+\lambda^{2}-1}{\prod_{i=1}^{n}\left(\left(\lambda+(1-\lambda)\left(1-\mu_{p(i)}^{q}\right)\right) /\left(1-\mu_{p(i)}^{q}\right)\right)^{\delta_{i}}-1}\right)^{1 / n !}, \\
& \nu^{\prime}=\prod_{p \in \mathbf{P}_{n}}\left(\frac{\prod_{i=1}^{n}\left(\left(\lambda+(1-\lambda) v_{p(i)}^{q}\right) / \nu_{p(i)}^{q}\right)^{\delta_{i}}+\lambda^{2}-1}{\prod_{i=1}^{n}\left(\left(\lambda+(1-\lambda) v_{p(i)}^{q}\right) / \nu_{p(i)}^{q}\right)^{\delta_{i}}-1}\right)^{1 / n !} .
\end{aligned}
$$

(9) If $f(t)=-\operatorname{In}\left[(\varepsilon-1) /\left(\varepsilon^{y}-1\right)\right] \quad\left(y=t^{q} ; \quad \varepsilon>1\right)$, then $g(t)=-\operatorname{In}\left[(\varepsilon-1) /\left(\varepsilon^{1-y}-1\right)\right], f^{-1}(t)=\left\{\log _{\varepsilon}\left[\left(\varepsilon-1+e^{-t}\right) /\right.\right.$ $\left.\left.e^{-t}\right]\right\}^{1 / q}, \quad$ and $\quad g^{-1}(t)=\left\{1-\log _{\varepsilon}\left[\left(\varepsilon-1+e^{-t}\right) / e^{-t}\right]\right\}^{1 / q}$.
According to equation (38), a q-rung orthopair fuzzy Archimedean Frank GMM (qROFAFGMM) operator is constructed as follows:

$\operatorname{qROFAFGMM}^{\Delta}\left(\Xi_{1}, \Xi_{2}, \ldots, \Xi_{n}\right)=\left\langle\left(1-\log _{\varepsilon}\left(1+\frac{\left(\varepsilon^{1-\mu^{\prime \prime}}-1\right)^{1 /\left(\sum_{i=1}^{n} \delta_{i}\right)}}{(\varepsilon-1)^{\left(1 /\left(\sum_{i=1}^{n} \delta_{i}\right)\right)-1}}\right)\right)^{1 / q},\left(\log _{\varepsilon}\left(1+\frac{\left(\varepsilon^{v^{\prime \prime}}-1\right)^{1 /\left(\sum_{i=1}^{n} \delta_{i}\right)}}{(\varepsilon-1)^{\left(1 /\left(\sum_{i=1}^{n} \delta_{i}\right)\right)-1}}\right)\right)^{1 / q}\right\rangle$, 
where

$$
\begin{aligned}
& \mu^{\prime \prime}=\log _{\varepsilon} \frac{\varepsilon-1+\left(\prod_{p \in \mathbf{P}_{n}}\left((\varepsilon-1) /\left(\varepsilon^{\mu^{\prime}}-1\right)\right)\right)^{1 / n !}}{\left(\prod_{p \in \mathbf{P}_{n}}\left((\varepsilon-1) /\left(\varepsilon^{\mu^{\prime}}-1\right)\right)\right)^{1 / n !}}, \\
& \nu^{\prime \prime}=1-\log _{\varepsilon} \frac{\varepsilon-1+\left(\prod_{p \in \mathbf{P}_{n}}\left((\varepsilon-1) /\left(\varepsilon^{1-\nu^{\prime}}-1\right)\right)\right)^{1 / n !}}{\left(\prod_{p \in \mathbf{P}_{n}}\left((\varepsilon-1) /\left(\varepsilon^{1-\nu^{\prime}}-1\right)\right)\right)^{1 / n !}}, \\
& \mu^{\prime}=1-\log _{\varepsilon} \frac{\varepsilon-1+\prod_{i=1}^{n}\left((\varepsilon-1) /\left(\varepsilon^{1-\mu_{p(i)}^{q}}-1\right)\right)^{\delta_{i}}}{\prod_{i=1}^{n}\left((\varepsilon-1) /\left(\varepsilon^{1-\mu_{p(i)}^{q}}-1\right)\right)^{\delta_{i}}}, \\
& \nu^{\prime}=\log _{\varepsilon} \frac{\varepsilon-1+\prod_{i=1}^{n}\left((\varepsilon-1) /\left(\varepsilon^{v_{p(i)}^{q}}-1\right)\right)^{\delta_{i}}}{\prod_{i=1}^{n}\left((\varepsilon-1) /\left(\varepsilon^{v_{p(i)}^{q}}-1\right)\right)^{\delta_{i}}} .
\end{aligned}
$$

3.2.2. qROFWAGMM Operators. The qROFAGMM operator has advantages in having desirable generality and flexibility and capturing the interrelationships among multiple aggregated qROFNs. But it does not consider the relative importance of each aggregated qROFN. To make up for this deficiency, weights are introduced and a
qROFWAGMM operator is presented. The formal definition of the presented operator is as follows:

Definition 11. Let $\left(\Xi_{1}, \Xi_{2}, \ldots, \Xi_{n}\right)\left(\Xi_{i}=<\mu_{i}, v_{i}>, i=1,2, \ldots\right.$, $n)$ be a collection of $n$ qROFNs $(q=1,2,3, \ldots), \Delta=\left(\delta_{1}, \delta_{2}\right.$, $\left.\ldots, \delta_{n}\right)\left(\delta_{1}, \delta_{2}, \ldots, \delta_{n} \geq 0\right.$ but not at the same time $\left.\delta_{1}=\delta_{2}=\ldots=\delta_{n}=0\right)$ be a collection of $n$ real numbers, $p(i)$ be a permutation of $(1,2, \ldots, n), \mathbf{P}_{n}$ be the set of all permutations of $(1,2, \ldots, n), \Xi_{i} \oplus \Xi_{j}$ and $\Xi_{i} \otimes \Xi_{j}(i, j=1,2, \ldots, n)$ be, respectively, the sum and product operations of $\Xi_{i}$ and $\Xi_{j}$ based on ATT, $\sigma \Xi_{r}$ and $\Xi_{s}^{\tau}(r, s=1,2, \ldots, n ; \sigma, \tau>0)$ be, respectively, the multiplication operation of $\Xi_{r}$ and the power operation of $\Xi_{s}$ based on ATT, and $w_{1}, w_{2}, \ldots, w_{n}$ be, respectively, the weights of $\Xi_{1}, \Xi_{2}, \ldots, \Xi_{n}$ such that $0 \leq w_{1}$, $w_{2}, \ldots, w_{n} \leq 1$ and $w_{1}+w_{2}+\ldots+w_{n}=1$. Then the aggregation function,

$$
\begin{gathered}
\operatorname{qROFWAGMM}^{\Delta}\left(\Xi_{1}, \Xi_{2}, \ldots, \Xi_{n}\right) \\
=\frac{1}{\sum_{i=1}^{n} \delta_{i}}\left(\underset{p \in \mathbf{P}_{n}}{\otimes} \underset{i=1}{\oplus}\left(\delta_{i} \Xi_{p(i)}^{n w_{p(i)}}\right)\right)^{1 / n !},
\end{gathered}
$$

is called the qROFWAGMM operator.

According to equations (1)-(4) and (50), the following theorem is obtained.

Theorem 10. Let $\left(\Xi_{1}, \Xi_{2}, \ldots, \Xi_{n}\right)\left(\Xi_{i}=<\mu_{i}, v_{i}>, i=1,2, \ldots\right.$, $n)$ be a collection of $n q R O F N s(q=1,2,3, \ldots)$. Then

$$
\begin{aligned}
\operatorname{qROFWAGMM}^{\Delta}\left(\Xi_{1}, \Xi_{2}, \ldots, \Xi_{n}\right)= & \left\langleg ^ { - 1 } \left(\frac{1}{\sum_{i=1}^{n} \delta_{i}} g\left(f^{-1}\left(\frac{1}{n !} \sum_{p \in \mathbf{P}_{n}} f\left(g^{-1}\left(\sum_{i=1}^{n}\left(\delta_{i} g\left(f^{-1}\left(\left(n w_{p(i)}\right) f\left(\mu_{p(i)}\right)\right)\right)\right)\right)\right)\right)\right)\right.\right. \\
& f^{-1}\left(\frac{1}{\sum_{i=1}^{n} \delta_{i}} f\left(g^{-1}\left(\frac{1}{n !} \sum_{p \in \mathbf{P}_{n}} g\left(f^{-1}\left(\sum_{i=1}^{n}\left(\delta_{i} f\left(g^{-1}\left(\left(n w_{p(i)}\right) g\left(\nu_{p(i)}\right)\right)\right)\right)\right)\right)\right)\right)\right),
\end{aligned}
$$

and $q \operatorname{ROFWAGMM}{ }^{\Delta}\left(\Xi_{1}, \Xi_{2}, \ldots, \Xi_{n}\right)$ is still a $q R O F N$.

For the details of the proof of this theorem, please refer to Appendix J. In addition, it is similar to prove that the qROFWAGMM operator has the properties of monotonicity and boundedness (please note that the qROFWAGMM operator no longer has idempotency).

Like equation (38), equation (51) is a generalized form of the qROFWAGMM operator. If specific values are assigned to $q$ and $\delta_{1}, \delta_{2}, \ldots, \delta_{n}$ and specific forms are assigned to $f$, then specific operators can be constructed:

(1) If $q=1$, then the qROFWAGMM operator will reduce to an intuitionistic fuzzy weighted Archimedean GMM operator.

(2) If $q=2$, then the qROFWAGMM operator will reduce to a Pythagorean fuzzy weighted Archimedean GMM operator.
(3) If $\delta_{1}=\delta>0$ and $\delta_{2}=\delta_{3}=\ldots=\delta_{n}=0$, then the qROFWAGMM operator will reduce to a q-rung orthopair fuzzy weighted Archimedean generalized geometric averaging operator.

(4) If $\delta_{1}, \delta_{2}>0$ and $\delta_{3}=\delta_{4}=\ldots=\delta_{n}=0$, then the qROFWAGMM operator will reduce to a q-rung orthopair fuzzy weighted Archimedean GBM operator.

(5) If $\delta_{1}=\delta_{2}=\ldots=\delta_{k}=1$ and $\delta_{k+1}=\delta_{k+2}=\ldots=\delta_{n}=0$, then the qROFWAGMM operator will reduce to a q-rung orthopair fuzzy weighted Archimedean GMSM operator.

(6) If $\delta_{1}=\delta_{2}=\ldots=\delta_{n}=\delta>0$, then the qROFWAGMM operator will reduce to a q-rung orthopair fuzzy weighted Archimedean generalized arithmetic averaging operator.

(7) If $f(t)=-\operatorname{In} t^{q}$, then $g(t)=-\operatorname{In}\left(1-t^{q}\right), f^{-1}(t)=\left(e^{-t}\right)^{1 / q}$, and $g^{-1}(t)=\left(1-e^{-t}\right)^{1 / q}$. According to equation (51), a 
q-rung orthopair fuzzy weighted Archimedean Algebraic GMM (qROFWAAGMM) operator is constructed as follows:

$$
\begin{aligned}
\operatorname{qROFWAAGMM}^{\Delta}\left(\Xi_{1}, \Xi_{2}, \ldots, \Xi_{n}\right)= & \left\langle\left(1-\left(1-\prod_{p \in \mathbf{P}_{n}}\left(1-\prod_{i=1}^{n}\left(1-\mu_{p(i)}^{q n w_{p(i)}}\right)^{\delta_{i}}\right)^{1 / n !}\right)^{1 / \Sigma_{i=1}^{n} \delta_{i}}\right)^{1 / q},\right. \\
& \left.\left(\left(1-\prod_{p \in \mathbf{P}_{n}}\left(1-\prod_{i=1}^{n}\left(1-\left(1-v_{p(i)}^{q}\right)^{n w_{p(i)}}\right)^{\delta_{i}}\right)^{1 / n !}\right)^{1 / \Sigma_{i=1}^{n} \delta_{i}}\right)^{1 / q}\right)^{1 / q} .
\end{aligned}
$$

This operator is actually the q-rung orthopair fuzzy weighted GMM operator presented by Wang et al. [50]. It has the following special cases:
(1) If $q=1$, then the qROFWAAGMM operator will reduce to

$\left\langle 1-\left(1-\prod_{p \in \mathbf{P}_{n}}\left(1-\prod_{i=1}^{n}\left(1-\mu_{p(i)}^{n w_{p(i)}}\right)^{\delta_{i}}\right)^{1 / n !},\left(1-\prod_{p \in \mathbf{P}_{n}}\left(1-\prod_{i=1}^{n}\left(1-\left(1-v_{p(i)}\right)^{n w_{p(i)}^{n} \delta_{i}}\right)^{\delta_{i}}\right)^{1 / n !}\right)^{1 / \Sigma_{i=1}^{n} \delta_{i}}\right)\right.$,

which is the intuitionistic fuzzy weighted GMM operator presented by Liu and Li [55].
(2) If $q=2$, then the qROFWAAGMM operator will reduce to

$$
\left\langle\left(1-\left(1-\prod_{p \in \mathbf{P}_{n}}\left(1-\prod_{i=1}^{n}\left(1-\mu_{p(i)}^{2 n w_{p(i)}}\right)^{\delta_{i}}\right)^{1 / n !}\right)^{1 / \Sigma_{i=1}^{n} \delta_{i}}\right)^{1 / 2},\left(\left(1-\prod_{p \in \mathbf{P}_{n}}\left(1-\prod_{i=1}^{n}\left(1-\left(1-v_{p(i)}^{2}\right)^{n w_{p(i)}}\right)^{\delta_{i}}\right)^{1 / n !}\right)^{1 / \Sigma_{i=1}^{n} \delta_{i}}\right)^{1 / 2}\right)^{1 / 2}
$$

which is the Pythagorean fuzzy weighted GMM operator presented by Zhu and Li [56].

(3) If $\delta_{1}=\delta>0$ and $\delta_{2}=\delta_{3}=\ldots=\delta_{n}=0$, then the qROFWAAGMM operator will reduce to the operator in equation (30).
(4) If $\delta_{1}, \delta_{2}>0$ and $\delta_{3}=\delta_{4}=\ldots=\delta_{n}=0$, then the qROFWAAGMM operator will reduce to

$$
\begin{aligned}
& \left\langle\left(1-\left(1-\prod_{\substack{i, j=1 \\
j \neq i}}^{n}\left(1-\left(1-\mu_{i}^{q n w_{i}}\right)^{\delta_{1}}\left(1-\mu_{j}^{q n w_{j}}\right)^{\delta_{2}}\right)^{1 / n(n-1)}\right)^{1 /\left(\delta_{1}+\delta_{2}\right)}\right)^{1 / q},\right. \\
& \left.\left(\left(1-\prod_{\substack{i, j=1 \\
j \neq i}}^{n}\left(1-\left(1-\left(1-v_{i}^{q}\right)^{n w_{i}}\right)^{\delta_{1}}\left(1-\left(1-v_{j}^{q}\right)^{n w_{j}}\right)^{\delta_{2}}\right)^{n(n-1)}\right)^{1 /\left(\delta_{1}+\delta_{2}\right)}\right)^{1 / q}\right\rangle,
\end{aligned}
$$


which is a q-rung orthopair fuzzy weighted Archimedean Algebraic GBM operator.
(5) If $\delta_{1}=\delta_{2}=\ldots=\delta_{k}=1$ and $\delta_{k+1}=\delta_{k+2}=\ldots=\delta_{n}=0$, then the qROFWAAGMM operator will reduce to

$$
\left\langle\left(1-\left(1-\prod_{1 \leq i_{1}<\ldots<i_{k} \leq n}^{n}\left(1-\prod_{j=1}^{k}\left(1-\mu_{i_{j}}^{q n w_{i_{j}}}\right)\right)^{k !(n-k) !}\right)^{1 / k}\right)^{1 / q},\left(\left(1-\prod_{1 \leq i_{1}<\ldots<i_{k} \leq n}^{n}\left(1-\prod_{j=1}^{k}\left(1-\left(1-v_{i_{j}}^{q}\right)^{n w_{i_{j}}}\right)\right)^{k !(n-k) ! n !}\right)^{1 / k}\right)^{1 / q}\right\rangle^{\prime}
$$

which is the q-rung orthopair fuzzy weighted GMSM operator presented by Wei et al. [47].

(6) If $\delta_{1}=\delta_{2}=\ldots=\delta_{n}=\delta>0$, then the qROFWAAGMM operator will reduce to the qROFWGAA operator in equation (27).
(7) If $f(t)=\operatorname{In}\left[\left(2-t^{q}\right) / t^{q}\right]$, then $g(t)=\operatorname{In}\left[\left(1+t^{q}\right) /\left(1-t^{q}\right)\right]$, $f^{-1}(t)=\left[2 /\left(\mathrm{e}^{t}+1\right)\right]^{1 / q}$, and $g^{-1}(t)=\left[\left(\mathrm{e}^{t}-1\right) /\left(\mathrm{e}^{t}+1\right)\right]^{1 /}$ $q$. According to equation (51), a q-rung orthopair fuzzy weighted Archimedean Einstein GMM (qROFWAEGMM) operator is constructed as follows:

$\operatorname{qROFWAEGMM}^{\Delta}\left(\Xi_{1}, \Xi_{2}, \ldots, \Xi_{n}\right)=\left\langle\left(\frac{\left(\mu^{\prime}+3\right)^{1 /\left(\sum_{i=1}^{n} \delta_{i}\right)}-\left(\mu^{\prime}-1\right)^{1 /\left(\Sigma_{i=1}^{n} \delta_{i}\right)}}{\left(\mu^{\prime}+3\right)^{1 /\left(\sum_{i=1}^{n} \delta_{i}\right)}+\left(\mu^{\prime}-1\right)^{1 /\left(\sum_{i=1}^{n} \delta_{i}\right)}}\right)^{1 / q},\left(\frac{2\left(\nu^{\prime}-1\right)^{1 /\left(\Sigma_{i=1}^{n} \delta_{i}\right)}}{\left(\nu^{\prime}+3\right)^{1 /\left(\Sigma_{i=1}^{n} \delta_{i}\right)}+\left(\nu^{\prime}-1\right)^{1 /\left(\sum_{i=1}^{n} \delta_{i}\right)}}\right)^{1 / q}\right\rangle$,

where

$$
\begin{aligned}
& \mu^{\prime}=\prod_{p \in \mathbf{P}_{n}}\left(\frac{\prod_{i=1}^{n}\left(\left(\left(2-\mu_{p(i)}^{q}\right)^{n w_{p(i)}}+3 \mu_{p(i)}^{q n w_{p(i)}}\right) /\left(\left(2-\mu_{p(i)}^{q}\right)^{n w_{p(i)}}-\mu_{p(i)}^{q n w_{p(i)}}\right)\right)^{\delta_{i}}+3}{\prod_{i=1}^{n}\left(\left(\left(2-\mu_{p(i)}^{q}\right)^{n w_{p(i)}}+3 \mu_{p(i)}^{q n w_{p(i)}}\right) /\left(\left(2-\mu_{p(i)}^{q}\right)^{n w_{p(i)}}-\mu_{p(i)}^{q n w_{p(i)}}\right)\right)^{\delta_{i}}-1},\right. \\
& \nu^{\prime}=\prod_{p \in \mathbf{P}_{n}}\left(\frac{\prod_{i=1}^{n}\left(\left(\left(1+v_{p(i)}^{q}\right)^{n w_{p(i)}}+3\left(1-v_{p(i)}^{q}\right)^{p(i)}\right) /\left(\left(1+v_{p(i)}^{q}\right)^{n w_{p(i)}}-\left(1-v_{p(i)}^{q}\right)^{n w_{p(i)}}\right)\right)^{\delta_{i}}+3}{\prod_{i=1}^{n}\left(\left(\left(1+v_{p(i)}^{q}\right)^{n w_{p(i)}}+3\left(1-v_{p(i)}^{q}\right)^{n w_{p(i)}}\right) /\left(\left(1+v_{p(i)}^{q}\right)^{n w_{p(i)}}-\left(1-v_{p(i)}^{q}\right)^{n w_{p(i)}}\right)\right)^{\delta_{i}}-1} .\right.
\end{aligned}
$$

(8) If $f(t)=\operatorname{In}\left\{\left[\lambda+(1-\lambda) t^{q}\right] / t^{q}\right\} \quad(\lambda>0)$, then $g(t)=\operatorname{In}$ $\left\{\left[\lambda+(1-\lambda)\left(1-t^{q}\right)\right] /\left(1-t^{q}\right)\right\}, f^{-1}(t)=\left[\lambda /\left(\mathrm{e}^{t}+\lambda-1\right)\right]^{1 / q}$, and $g^{-1}(t)=\left[\left(e^{t}-1\right) /\left(e^{t}+\lambda-1\right)\right]^{1 / q}$. According to equation (51), a q-rung orthopair fuzzy weighted Archimedean Hamacher GMM (qROFWAHGMM) operator is constructed as follows:

$$
\begin{aligned}
\operatorname{qROFWAHGMM}^{\Delta}\left(\Xi_{1}, \Xi_{2}, \ldots, \Xi_{n}\right)= & \left\langle\left(\frac{\left(\mu^{\prime}+\lambda^{2}-1\right)^{1 /\left(\sum_{i=1}^{n} \delta_{i}\right)}-\left(\mu^{\prime}-1\right)^{1 /\left(\sum_{i=1}^{n} \delta_{i}\right)}}{\left(\mu^{\prime}+\lambda^{2}-1\right)^{1 /\left(\Sigma_{i=1}^{n} \delta_{i}\right)}+(\lambda-1)\left(\mu^{\prime}-1\right)^{1 /\left(\Sigma_{i=1}^{n} \delta_{i}\right)}}\right)^{1 / q},\right. \\
& \left.\left(\frac{\lambda\left(\nu^{\prime}-1\right)^{1 /\left(\Sigma_{i=1}^{n} \delta_{i}\right)}}{\left(\nu^{\prime}+\lambda^{2}-1\right)^{1 /\left(\Sigma_{i=1}^{n} \delta_{i}\right)}+(\lambda-1)\left(\nu^{\prime}-1\right)^{1 /\left(\Sigma_{i=1}^{n} \delta_{i}\right)}}\right)^{1 / q}\right\rangle,
\end{aligned}
$$

where 


$$
\begin{aligned}
& \mu^{\prime}=\prod_{p \in \mathbf{P}_{n}}\left(\frac{\prod_{i=1}^{n}\left(\left(\left(\lambda+(1-\lambda) \mu_{p(i)}^{q}\right)^{n w_{p(i)}}+\left(\lambda^{2}-1\right) \mu_{p(i)}^{q n w_{p(i)}}\right) /\left(\left(\lambda+(1-\lambda) \mu_{p(i)}^{q}\right)^{n w_{p(i)}}-\mu_{p(i)}^{q n w_{p(i)}}\right)\right)^{\delta_{i}}+\lambda^{2}-1}{\prod_{i=1}^{n}\left(\left(\left(\lambda+(1-\lambda) \mu_{p(i)}^{q}\right)^{n w_{p(i)}}+\left(\lambda^{2}-1\right) \mu_{p(i)}^{q n w_{p(i)}}\right) /\left(\left(\lambda+(1-\lambda) \mu_{p(i)}^{q}\right)^{n w_{p(i)}}-\mu_{p(i)}^{q n w_{p(i)}}\right)\right)^{\delta_{i}}-1},\right. \\
& \nu^{\prime}=\prod_{p \in \mathbf{P}_{n}}\left(\frac{\left.\prod_{i=1}^{n}\left(\left(\left(\lambda+(1-\lambda)\left(1-v_{p(i)}^{q}\right)\right)^{n w_{p(i)}}+\left(\lambda^{2}-1\right)\left(1-v_{p(i)}^{q}\right)^{n w_{p(i)}}\right) /\left(\left(\lambda+(1-\lambda)\left(1-v_{p(i)}^{q}\right)\right)^{n w_{p(i)}}-\left(1-v_{p(i)}^{q}\right)\right)^{n w_{p(i)}}\right)\right)^{\delta_{i}}+\lambda^{2}-1}{\prod_{i=1}^{n}\left(\left(\left(\lambda+(1-\lambda)\left(1-v_{p(i)}^{q}\right)\right)^{n w_{p(i)}}+\left(\lambda^{2}-1\right)\left(1-v_{p(i)}^{q}\right)^{n w_{p(i)}}\right) /\left(\left(\lambda+(1-\lambda)\left(1-v_{p(i)}^{q}\right)\right)^{n w_{p(i)}}-\left(1-v_{p(i)}^{q}\right)\right.\right.} .\right.
\end{aligned}
$$

(9) If $f(t)=-\operatorname{In}\left[(\varepsilon-1) /\left(\varepsilon^{y}-1\right)\right] \quad\left(y=t^{q} ; \quad \varepsilon>1\right)$, then $g(t)=-\operatorname{In}\left[(\varepsilon-1) /\left(\varepsilon^{1-y}-1\right)\right], f^{-1}(t)=\left\{\log _{\varepsilon}\left[\left(\varepsilon-1+e^{-t}\right) /\right.\right.$ $\left.\left.e^{-t}\right]\right\}^{1 / q}, \quad$ and $\quad g^{-1}(t)=\left\{1-\log _{\varepsilon}\left[\left(\varepsilon-1+e^{-t}\right) / e^{-t}\right]\right\}^{1 / q}$.
According to equation (51), a q-rung orthopair fuzzy weighted Archimedean Frank GMM (qROFWAFGMM) operator is constructed as follows:

$\operatorname{qROFWAFGMM}^{\Delta}\left(\Xi_{1}, \Xi_{2}, \ldots, \Xi_{n}\right)=\left\langle\left(1-\log _{\varepsilon}\left(1+\frac{(\varepsilon-1)\left(\varepsilon^{1-\mu^{\prime \prime}}-1\right)^{1 /\left(\sum_{i=1}^{n} \delta_{i}\right)}}{(\varepsilon-1)^{1 /\left(\sum_{i=1}^{n} \delta_{i}\right)}}\right)\right)^{1 / q},\left(\log _{\varepsilon}\left(1+\frac{(\varepsilon-1)\left(\varepsilon^{\varepsilon^{\prime \prime \prime}}-1\right)^{1 /\left(\sum_{i=1}^{n} \delta_{i}\right)}}{(\varepsilon-1)^{1 /\left(\sum_{i=1}^{n} \delta_{i}\right)}}\right)\right)^{1 / q}\right\rangle$,

where

$$
\begin{aligned}
& \mu^{\prime \prime \prime}=\log _{\varepsilon} \frac{\varepsilon-1+\prod_{p \in \mathbf{P}_{n}}\left((\varepsilon-1) /\left(\varepsilon^{\mu^{\prime \prime}}-1\right)\right)^{1 / n !}}{\prod_{p \in \mathbf{P}_{n}}\left((\varepsilon-1) /\left(\varepsilon^{\mu^{\prime \prime}}-1\right)\right)^{1 / n !}}, \\
& \nu^{\prime \prime \prime}=1-\log _{\varepsilon} \frac{\varepsilon-1+\prod_{p \in \mathbf{P}_{n}}\left((\varepsilon-1) /\left(\varepsilon^{1-\nu^{\prime \prime}}-1\right)\right)^{1 / n !}}{\prod_{p \in \mathbf{P}_{n}}\left((\varepsilon-1) /\left(\varepsilon^{1-\nu^{\prime \prime}}-1\right)\right)^{1 / n !}} \\
& \mu^{\prime \prime}=1-\log _{\varepsilon} \frac{\varepsilon-1+\prod_{i=1}^{n}\left((\varepsilon-1) /\left(\varepsilon^{1-\mu^{\prime}}-1\right)\right)^{\delta_{i}}}{\prod_{i=1}^{n}\left((\varepsilon-1) /\left(\varepsilon^{1-\mu^{\prime}}-1\right)\right)^{\delta_{i}}}, \\
& v^{\prime \prime}=\log _{\varepsilon} \frac{\varepsilon-1+\prod_{i=1}^{n}\left((\varepsilon-1) /\left(\varepsilon^{\nu^{\prime}}-1\right)\right)^{\delta_{i}}}{\prod_{i=1}^{n}\left((\varepsilon-1) /\left(\varepsilon^{\nu^{\prime}}-1\right)\right)^{\delta_{i}}}, \\
& \mu^{\prime}=\log _{\varepsilon}\left(1+\frac{\left(\varepsilon^{\mu_{p(i)}^{q}}-1\right)^{n w_{p(i)}}}{(\varepsilon-1)^{n w_{p(i)}-1}}\right) \\
& \nu^{\prime}=1-\log _{\varepsilon}\left(1+\frac{\left(\varepsilon^{1-v_{p(i)}^{q}}-1\right)^{n w_{p(i)}}}{(\varepsilon-1)^{n w_{p(i)}-1}}\right) .
\end{aligned}
$$

\section{MCGDM Method}

In this section, a MCGDM method based on the qROFWAMM operator and the qROFWAGMM operator is proposed to solve the MCGDM problems based on qROFNs.

Generally, a MCGDM problem based on qROFNs can be described via a set of alternatives $\mathbf{A}=\left\{A_{1}, A_{2}, \ldots, A_{m}\right\}$, a set of criteria $\mathbf{C}=\left\{C_{1}, C_{2}, \ldots, C_{n}\right\}$, a vector of weights of criteria $\mathbf{w}=\left[w_{1}, \quad w_{2}, \ldots, \quad w_{n}\right] \quad\left(0 \leq w_{1}, \quad w_{2}, \ldots, \quad w_{n} \leq 1\right.$, $w_{1}+w_{2}+\ldots+w_{n}=1$, and each element, respectively, stands for the relative importance of the criteria $C_{1}, C_{2}, \ldots, C_{n}$ ), a set of experts $\mathbf{E}=\left\{E_{1}, E_{2}, \ldots, E_{N}\right\}$, a vector of weights of experts $\boldsymbol{\varpi}=\left[\begin{array}{llll}\varpi_{1}, & \omega_{2}, \ldots, \omega_{N}\end{array}\right] \quad\left(0 \leq \varpi_{1}, \varpi_{2}, \ldots, \varpi_{N} \leq 1\right.$, $\omega_{1}+\omega_{2}+\ldots+\Phi_{N}=1$, and each element, respectively, stands for the relative importance of the expert $\left.E_{1}, E_{2}, \ldots, E_{N}\right)$, and $N$ q-rung orthopair fuzzy decision matrices $\mathbf{M}_{k}=\left[\Xi_{k, i, j}\right]_{m \times n}$ $\left(k=1,2, \ldots, N ; i=1,2, \ldots, m ; j=1,2, \ldots, n ; \Xi_{k, i, j}=\left\langle\mu_{k, i, j}\right.\right.$, $v_{k, i, j}>$ is a qROFN that stands for the evaluation value of criterion $C_{j}$ with respect to alternative $A_{i}$ provided by expert $\left.E_{k}\right)$. Based on these components, the MCGDM problem can be described as follows: determining the optimal alternative with the help of a ranking of the elements of $\mathbf{A}$ based on $\mathbf{M}_{k}$, $\mathbf{w}$, and $\boldsymbol{\sigma}$. Using the qROFWAMM operator or the qROFWAGMM operator, the problem is solved according to the following steps:

(1) Normalize the q-rung orthopair fuzzy decision matrices. In general, a MCGDM problem may contain two different types of criteria, i.e., benefit and cost criteria. They affect the aggregation results positively and negatively, respectively. To eliminate 
such effects, the q-rung orthopair fuzzy decision matrices $\mathbf{M}_{k}=\left[\Xi_{k, i, j}\right]_{m \times n}$ are normalized as

$$
\mathbf{M}_{\mathbf{N}, k}= \begin{cases}{\left[\left\langle\mu_{k, i, j}, v_{k, i, j}\right\rangle\right]_{m \times n^{\prime}}} & \text { if } C_{j} \text { is a benefit criterion, } \\ {\left[\left\langle v_{k, i, j}, \mu_{k, i, j}\right\rangle\right]_{m \times n},} & \text { if } C_{j} \text { isa cost criterion. }\end{cases}
$$

(2) Calculate the collective evaluation value of each criterion of each alternative. Taking the normalized q-rung orthopair fuzzy decision matrices $\mathbf{M}_{\mathbf{N}, k}$ and the expert weight vector $\boldsymbol{\varpi}$ as input, the collective evaluation value of criterion $C_{j}$ of alternative $A_{i}$ is computed using the following equation:

$$
\begin{aligned}
\Xi_{i, j}= & \left\langle\mu_{i, j}, v_{i, j}\right\rangle=\text { qROFWAMM }^{\Delta} \\
& \cdot\left(\Xi_{1, i, j}, \Xi_{2, i, j}, \ldots, \Xi_{N, i, j}\right),
\end{aligned}
$$

or

$$
\begin{aligned}
\Xi_{i, j}= & \left\langle\mu_{i, j}, v_{i, j}\right\rangle=\text { qROFWAGMM }^{\Delta} \\
& \cdot\left(\Xi_{1, i, j}, \Xi_{2, i, j}, \ldots, \Xi_{N, i, j}\right),
\end{aligned}
$$

where qROFWAMM is an arbitrary specific qROFWAMM operator, such as the qROFWAAMM operator in equation (24), the qROFWAEMM operator in equation (31), the qROFWAHMM operator in equation (33), and the qROFWAFMM operator in equation (35); qROFWAGMM is an arbitrary specific qROFWAGMM operator, such as the qROFWAAGMM operator in equation (52), the qROFWAEGMM operator in equation (57), the qROFWAHGMM operator in equation (59), and the qROFWAFGMM operator in equation (61); and the values of the elements in $\Delta=\left(\delta_{1}, \delta_{2}, \ldots, \delta_{n}\right)$ are determined via judging whether the evaluation results of all experts are mutually independent, there are interrelationships between the evaluation results of any two experts, or there are interrelationships among the evaluation results of any three or more experts. Generally, the evaluation results of all experts should be independent. Therefore, $\delta_{1}>0$ and $\delta_{2}=\delta_{3}=\ldots=\delta_{n}=0$.

(3) Establish a collective q-rung orthopair fuzzy decision matrix. According to the collective evaluation value of each criterion of each alternative $\Xi_{i, j}$, the collective q-rung orthopair fuzzy decision matrix is established as $\mathbf{M}=\left[\Xi_{i, j}\right]_{m \times n}$.

(4) Calculate the collective overall preference of each alternative. Taking the collective q-rung orthopair fuzzy decision matrix $\mathbf{M}$ and the criterion weight vector $\mathbf{w}$ as input, the collective overall preference of alternative $A_{i}$ is computed using the following equation:

$$
\Xi_{i}=\left\langle\mu_{i}, v_{i}\right\rangle=\operatorname{qROFWAMM}^{\Delta}\left(\Xi_{i, 1}, \Xi_{i, 2}, \ldots, \Xi_{i, n}\right),
$$

$$
\begin{aligned}
& \text { or } \\
& \Xi_{i}=\left\langle\mu_{i}, v_{i}\right\rangle=\operatorname{qROFWAGMM}^{\Delta}\left(\Xi_{i, 1}, \Xi_{i, 2}, \ldots, \Xi_{i, n}\right),
\end{aligned}
$$

where qROFWAMM is the same specific qROFWAMM operator used in equation (64), qROFWAGMM is the same specific qROFWAGMM operator used in equation (65), and the values of the elements in $\Delta=\left(\delta_{1}, \delta_{2}, \ldots, \delta_{n}\right)$ are determined via judging whether all criteria are mutually independent, there are interrelationships between any two criteria, or there are interrelationships among any three or more criteria. When all criteria are mutually independent, then $\delta_{1}>0$ and $\delta_{2}=\delta_{3}=\ldots=\delta_{n}=0$; when there are interrelationships between any two criteria, $\delta_{1}$, $\delta_{2}>0$ and $\delta_{3}=\delta_{4}=\ldots=\delta_{n}=0$; and when there are interrelationships among any $r(r=3,4, \ldots, n)$ criteria, $\quad \delta_{1}, \quad \delta_{2}, \quad \ldots, \quad \delta_{r}>0$ and $\delta_{r+1}=$ $\delta_{r+2}=\ldots=\delta_{n}=0$.

(5) Calculate the score and accuracy of the collective overall preference of each alternative. The score and accuracy of the collective overall preference of alternative $A_{i}$ is, respectively, computed using the equations in Definitions 2 and 3.

(6) Generate a ranking of all alternatives and determine the optimal one. On the basis of the scores and accuracies of the collective overall preferences of all alternatives, a ranking of these alternatives can be generated according to the comparison rules in Definition 4. The optimal alternative is determined with the help of the ranking.

\section{Example, Experiments, and Comparisons}

In this section, a numerical example is firstly leveraged to illustrate the working process of the proposed MCGDM method. Then a set of experiments are carried out to explore the effects of different specific operators and different parameter values on the aggregation results. Finally, qualitative and quantitative comparisons between the proposed method and some existing methods are made to demonstrate the feasibility and effectiveness of the proposed method.

5.1. Example. A numerical example about the determination of the best company for investment from five possible companies (cited from Reference [58]) is used to demonstrate the proposed MCGDM method. In this example, the five possible companies are $A_{1}, A_{2}, A_{3}, A_{4}$, and $A_{5}$. There are four determination criteria of these companies, which are the risk analysis $\left(C_{1}\right)$, the growth analysis $\left(C_{2}\right)$, the socialpolitical impact analysis $\left(C_{3}\right)$, and the environmental impact analysis $\left(C_{4}\right)$. The relative importance of these criteria is measured by the weight vector $\mathbf{w}=[0.2,0.1,0.3,0.4]$. The evaluation group consists of three different experts $E_{1}, E_{2}$, and $E_{3}$ whose relative importance is quantified by the weight 
vector $\boldsymbol{\varpi}=[0.35,0.40,0.25]$. To provide more freedom in the evaluation of the values of the four criteria of each company, experts were allowed to use qROFNs. The evaluation results of the three experts are, respectively, given in the following three matrices:

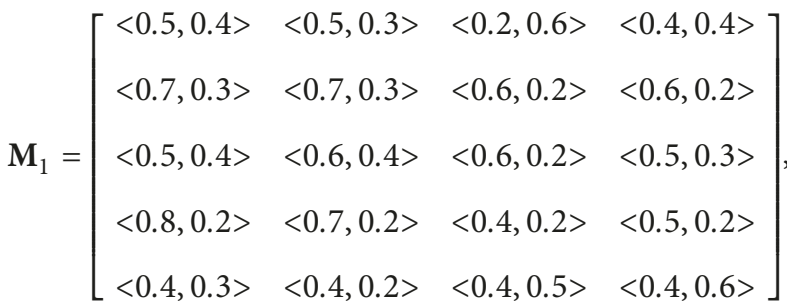

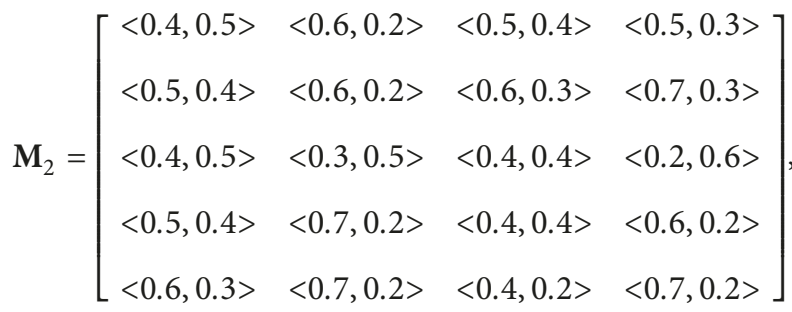

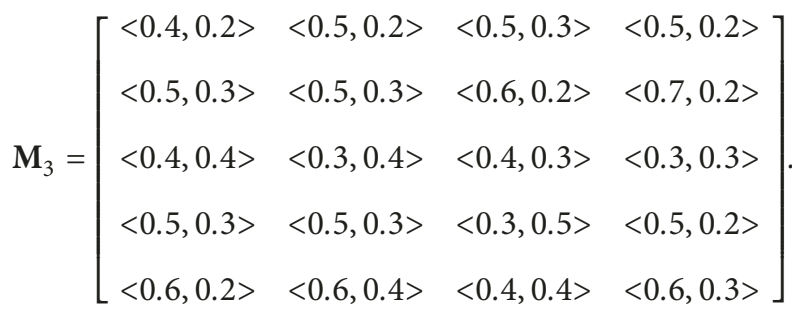

With the q-rung orthopair fuzzy decision matrices $\mathbf{M}_{1}, \mathbf{M}_{2}$, and $\mathbf{M}_{3}$, the expert weight vector $\boldsymbol{\varpi}$, and the criterion weight vector $\mathbf{w}$, the determination of the best company for investment can be carried out using the proposed MCGDM method. The process of the determination consists of the following six steps:

(i) Normalize the q-rung orthopair fuzzy decision matrices. Since the four criteria are all benefit criteria, normalization is not required. In other words, each normalized q-rung orthopair fuzzy decision matrix $\mathbf{M}_{\mathbf{N}, k}(k=1,2,3)$ is equal to $\mathbf{M}_{k}$, i.e., $\mathbf{M}_{\mathbf{N}, k}=\mathbf{M}_{k}$.

(ii) Calculate the collective evaluation value of each criterion of each company. Taking $\mathbf{M}_{\mathbf{N}, k}$ and $\boldsymbol{\varpi}$ as input, the collective evaluation value of $C_{j}(j=1,2,3$, $4)$ of $A_{i}(i=1,2,3,4,5)$ can be computed according to equation (64) or equation (65). Without loss of generality, the qROFWAHMM operator in equation (33) and the qROFWAHGMM operator in equation (59) (it is worth nothing that if $\lambda=1$ and $\lambda=2$, the Hamacher operators will reduce to the Algebraic and Einstein operators, respectively) are, respectively, used in equations (64) and (65).

(a) When the qROFWAHMM operator (it is assumed that the evaluation results of the three experts are independent. Therefore, $\Delta$ is assigned as $\Delta=\left(\delta_{1}, \delta_{2}, \delta_{3}\right)=(1,0,0)$. Further, $\lambda$ and $q$ are, respectively, assigned as $\lambda=3$ and $q=1$ ) is used, the computed results are as follows:

$\Xi_{1,1}=<0.4359,0.3748>, \Xi_{1,2}=<0.5415,0.2313>$, $\Xi_{1,3}=<0.3994,0.4357>$, and $\Xi_{1,4}=<0.4659$, $0.3024>$

$\Xi_{2,1}=<0.5774,0.3376>, \Xi_{2,2}=<0.6149,0.2561>$, $\Xi_{2,3}=<0.6000,0.2361>$, and $\Xi_{2,4}=<0.6673$, $0.2361>$

$\Xi_{3,1}=<0.4359,0.4385>, \Xi_{3,2}=<0.4127,0.4385>$, $\Xi_{3,3}=<0.4745,0.2952>$, and $\Xi_{3,4}=<0.3331$, $0.4052>$

$\left.\Xi_{4,1}=<0.6270,0.2952>, \Xi_{4,2}=<0.6562,0.2220\right\rangle$, $\Xi_{4,3}=<0.3754,0.3385>$, and $\Xi_{4,4}=<0.5415$, $0.2000>$

$\Xi_{5,1}=<0.5348,0.2719>, \Xi_{5,2}=<0.5815,0.2403>$, $\Xi_{5,3}=<0.4000,0.3356>$, and $\Xi_{5,4}=<0.5815$, $0.3374>$

(b) When the qROFWAHGMM operator $\left(\Delta=\left(\delta_{1}\right.\right.$, $\left.\delta_{2}, \delta_{3}\right)=(1,0,0), \lambda=3$, and $\left.q=1\right)$ is used, the computed results are as follows:

$\Xi_{1,1}=<0.4335,0.3929>, \quad \Xi_{1,2}=<0.5391, \quad 0.2350>$, $\Xi_{1,3}=<0.3730,0.4511>$, and $\Xi_{1,4}=<0.4636,0.3106>$ $\Xi_{2,1}=<0.5673,0.3404>, \Xi_{2,2}=<0.6084,0.2600>$, $\Xi_{2,3}=<0.6000,0.2400>$, and $\Xi_{2,4}=<0.6646$, $0.2400>$

$\Xi_{3,1}=<0.4335,0.4409>, \Xi_{3,2}=<0.3909,0.4409>$, $\Xi_{3,3}=<0.4652,0.3057>$, and $\Xi_{3,4}=<0.3118$, $0.4283>$

$\Xi_{4,1}=<0.6006,0.3057>, \Xi_{4,2}=<0.6481,0.2250>$, $\Xi_{4,3}=<0.3731,0.3575>$, and $\Xi_{4,4}=<0.5391$, $0.2000>$

$\Xi_{5,1}=<0.5255,0.2750>, \Xi_{5,2}=<0.5632,0.2505>$, $\Xi_{5,3}=<0.4000,0.3582>$, and $\Xi_{5,4}=<0.5632$, $0.3741>$

(iii) Establish a collective q-rung orthopair fuzzy decision matrix. According to the calculated $\Xi_{i, j}$, the collective q-rung orthopair fuzzy decision matrix can be established:

(a) When qROFWAHMM is used, the collective q-rung orthopair fuzzy decision matrix is as follows: 


$$
\mathbf{M}_{\mathrm{MM}}=\left[\begin{array}{cccc}
<0.4359,0.3748> & <0.5415,0.2313> & <0.3994,0.4357> & <0.4659,0.3024> \\
<0.5774,0.3376> & <0.6149,0.2561> & <0.6000,0.2361> & <0.6673,0.2361> \\
<0.4359,0.4385> & <0.4127,0.4385> & <0.4745,0.2952> & <0.3331,0.4052> \\
<0.6270,0.2952> & <0.6562,0.2220> & <0.3754,0.3385> & <0.5415,0.2000> \\
<0.5348,0.2719> & <0.5815,0.2403> & <0.4000,0.3356> & <0.5815,0.3374>
\end{array}\right] .
$$

(b) When qROFWAHGMM is used, the collective q-rung orthopair fuzzy decision matrix is as follows:

$$
\mathbf{M}_{\mathrm{GMM}}=\left[\begin{array}{llll}
<0.4335,0.3929> & <0.5391,0.2350> & <0.3730,0.4511> & <0.4636,0.3106> \\
<0.5673,0.3404> & <0.6084,0.2600> & <0.6000,0.2400> & <0.6646,0.2400> \\
<0.4335,0.4409> & <0.3909,0.4409> & <0.4652,0.3057> & <0.3118,0.4283> \\
<0.6006,0.3057> & <0.6481,0.2250> & <0.3731,0.3575> & <0.5391,0.2000> \\
<0.5255,0.2750> & <0.5632,0.2505\rangle & <0.4000,0.3582> & <0.5632,0.3741>
\end{array}\right] .
$$

(iv) Calculate the collective overall preference of each company. Taking $\mathbf{M}_{\mathrm{MM}}$ or $\mathbf{M}_{\mathrm{GMM}}$ and $\mathbf{w}$ as input, the collective overall preference of $A_{i}$ can be computed according to equation (66) or (67). Since qROFWAHMM and qROFWAHGMM are, respectively, used in the second step, they are, respectively, used in equations (66) and (67) to calculate the collective overall preference of $A_{i}$ :

(a) When qROFWAHMM (it is assumed that there are interrelationships among the four criteria. Therefore, $\Delta$ is assigned as $\Delta=\left(\delta_{1}, \delta_{2}, \delta_{3}\right.$, $\left.\delta_{4}\right)=(1,2,3,4)$. Further, $\lambda$ and $q$ are, respectively, assigned as $\lambda=3$ and $q=1$ ) is used, the computed results are as follows:

$\Xi_{1}=<0.4424,0.3722>, \quad \Xi_{2}=<0.5991,0.2999>$, $\Xi_{3}=<0.4030,0.3995>, \quad \Xi_{4}=<0.5170,0.3065>$, and $\Xi_{5}=<0.5047,0.3299>$

(b) When qROFWAHGMM $\left(\Delta=\left(\delta_{1}, \quad \delta_{2}, \quad \delta_{3}\right.\right.$, $\left.\delta_{4}\right)=(1,2,3,4), \lambda=3$, and $\left.q=1\right)$ is used, the computed results are as follows:

$\Xi_{1}=<0.4493,0.3558>, \quad \Xi_{2}=<0.6213,0.2601>$, $\Xi_{3}=<0.4179,0.3903>, \quad \Xi_{4}=<0.5235,0.2670>$, and $\Xi_{5}=<0.5121,0.3274>$

(v) Calculate the score and accuracy of the collective overall preference of each company. According to the calculated $\Xi_{i}$, the score and accuracy of the collective overall preference of $A_{i}$ can be computed using the equations in Definitions 2 and 3:

(a) When qROFWAHMM is used, the score and accuracy of $\Xi_{i}$ are, respectively, as follows:

$S\left(\Xi_{1}\right)=0.0702, \quad S\left(\Xi_{2}\right)=0.2992, \quad S\left(\Xi_{3}\right)=0.0034$,

$S\left(\Xi_{4}\right)=0.2105$, and $S\left(\Xi_{5}\right)=0.1748$

$A\left(\Xi_{1}\right)=0.8146, A\left(\Xi_{2}\right)=0.8990, A\left(\Xi_{3}\right)=0.8025$, $A\left(\Xi_{4}\right)=0.8235$, and $A\left(\Xi_{5}\right)=0.8346$

(b) When qROFWAHGMM is used, the score and accuracy of $\Xi_{i}$ are, respectively, as follows: $S\left(\Xi_{1}\right)=0.0934, \quad S\left(\Xi_{2}\right)=0.3612, \quad S\left(\Xi_{3}\right)=0.0276$, $S\left(\Xi_{4}\right)=0.2565$, and $S\left(\Xi_{5}\right)=0.1847$

$$
\begin{aligned}
& A\left(\Xi_{1}\right)=0.8051, A\left(\Xi_{2}\right)=0.8814, A\left(\Xi_{3}\right)=0.8082, \\
& A\left(\Xi_{4}\right)=0.7905, \text { and } A\left(\Xi_{5}\right)=0.8394
\end{aligned}
$$

(vi) Generate a ranking of all companies and determine the optimal one. On the basis of the calculated $S\left(\Xi_{i}\right)$ and $A\left(\Xi_{i}\right)$, a ranking of the five companies can be generated according to the comparison rules in Definition 4:

(a) When qROFWAHMM is used, the generated ranking is $A_{2}>A_{4}>A_{5}>A_{1}>A_{3}$

(b) When qROFWAHGMM is used, the generated ranking is $A_{2}>A_{4}>A_{5}>A_{1}>A_{3}$

With the help of the generated ranking, the optimal company is determined as company $A_{2}$.

5.2. Experiments. To explore the effect of using different groups of specific operators and assigning different values to parameters on the aggregation results, the following four experiments were carried out.

5.2.1. Experiment 1. It was carried out to show the influence of the use of different groups of specific operators on the aggregation results. In this experiment, $\mathbf{M}_{\mathbf{N} k}(k=1,2,3), \boldsymbol{\varpi}$, and $w$ in the example were used as the input of the presented eight groups of Archimedean Muirhead aggregation operators (when adapting the eight groups of operators, $\Delta=\left(\delta_{1}\right.$, $\left.\delta_{2}, \delta_{3}\right)=(1,0,0)$ (for equation (64) or equation (65)), $\Delta=\left(\delta_{1}\right.$, $\left.\delta_{2}, \delta_{3}, \delta_{4}\right)=(1,2,3,4)$ (for equation (66) or equation (67)), $\lambda=\varepsilon=3$, and $q=1)$. The results of the experiment are the calculated scores of the collective overall preferences of the five companies and the generated rankings of the five companies, which are listed in Table 1. As can be seen from the table, there are slight differences among the scores of the same company calculated by the four groups of weighted Archimedean MM operators or the four groups of weighted Archimedean GMM operators. But the scores of each group of GMM operators are obviously greater than the scores of the corresponding group of MM operators. 
TABLE 1: The results of Experiment 1.

\begin{tabular}{|c|c|c|c|c|c|c|c|}
\hline \multirow{2}{*}{$\begin{array}{l}\text { Specific operator used in equation } \\
\text { (64) or equation }(65)\end{array}$} & \multirow{2}{*}{$\begin{array}{l}\text { Specific operator used in equation } \\
(66) \text { or equation }(67)\end{array}$} & \multicolumn{5}{|c|}{$\begin{array}{l}\text { Calculated scores of the collective overall } \\
\text { preferences of the five companies }\end{array}$} & \multirow[t]{2}{*}{ Generated ranking } \\
\hline & & $S_{1}$ & $S_{2}$ & $S_{3}$ & $S_{4}$ & $S_{5}$ & \\
\hline qROFWAAMM & & 0.0431 & 0.2400 & -0.0460 & 0.1834 & 0.1430 & $A_{2}>A_{4}>A_{5}>A_{1}>A_{3}$ \\
\hline qROFWAEMM & qROFWAEMM & 0.0554 & 0.2718 & -0.0172 & 0.1950 & 0.1580 & $A_{2}>A_{4}>A_{5}>A_{1}>A_{3}$ \\
\hline qROFWAHMM & qROFWAHMM & 0.0702 & 0.2992 & 0.0034 & 0.2105 & 0.1748 & $A_{2}>A_{4}>A_{5}>A_{1}>A_{3}$ \\
\hline qROFWAFMM & qROFWAFMM & 0.0525 & 0.2636 & -0.0227 & 0.1913 & 0.1541 & $A_{2}>A_{4}>A_{5}>A_{1}>A_{3}$ \\
\hline qROFWAAGMM & qROFWAAGMM & 0.1508 & 0.3815 & 0.0698 & 0.3077 & 0.2321 & $A_{2}>A_{4}>A_{5}>A_{1}>A_{3}$ \\
\hline qROFWAEGMM & qROFWAEGMM & 0.1155 & 0.3696 & 0.0464 & 0.2760 & 0.2033 & $A_{2}>A_{4}>A_{5}>A_{1}>A_{3}$ \\
\hline qROFWAHGMM & qROFWAHGMM & 0.0934 & 0.3612 & 0.0276 & 0.2565 & 0.1847 & $A_{2}>A_{4}>A_{5}>A_{1}>A_{3}$ \\
\hline qROFWAFGMM & qROFWAFGMM & 0.1217 & 0.3712 & 0.0510 & 0.2812 & 0.2076 & $A_{2}>A_{4}>A_{5}>A_{1}>A_{3}$ \\
\hline
\end{tabular}

This indicates that the GMM operators tend to provide optimistic expectations, while the MM operators tend to generate pessimistic results relatively. Nevertheless, the rankings remain the same with respect to the eight groups of specific operators. This suggests that the use of different groups of specific operators has no obvious effect on the aggregation results.

5.2.2. Experiment 2. It was carried out to show the influence of the assignment of different values to the rung $q$ on the aggregation results. In this experiment, $\mathbf{M}_{M M}$ and $\mathbf{w}$ and $\mathbf{M}_{\mathrm{GMM}}$ and $\mathbf{w}$ in the example were, respectively, used as the input of the qROFWAHMM operator $\left(\Delta=\left(\delta_{1}, \delta_{2}, \delta_{3}\right.\right.$, $\left.\delta_{4}\right)=(1,2,3,4), \lambda=3$, and $\left.q=1,2, \ldots, 10\right)$ in equation (66) and the qROFWAHGMM operator $\left(\Delta=\left(\delta_{1}, \delta_{2}, \delta_{3}, \delta_{4}\right)=(1\right.$, $2,3,4), \lambda=3$ and $q=1,2, \ldots, 10$ ) in equation (67) (it is worth nothing that only the most generalized Hamacher operators were included in this and the subsequent experiments for the sake of simplicity). The results of the experiment are the calculated scores of the collective overall preferences of the five companies, which are depicted in Figure 1. From the figure, it can be found that the scores and ranking of the five companies will change as the value of $q$ changes. When $q=1$, $2,3,4,5$, the rankings generated by the two operators are all $A_{2}>A_{4}>A_{5}>A_{1}>A_{3}$. The best company has always been $A_{2}$. When $q=6,7,8,9,10$, the rankings generated by qROFWAHMM are difficult to distinguish, and the rankings generated by qROFWAHGMM are all $A_{4}>A_{2}>A_{5}>A_{1}>A_{3}$. The best company has changed to $A_{4}$. Obviously, if $q=1$, the qROFNs will become IFNs; if $q=2$, the qROFNs will become PFNs. From the experiment results in Figure 1, it is recommended that the smallest $q$ that can satisfy $0 \leq \mu^{q}+\nu^{q} \leq 1$ is assigned in practical applications. For example, if the value of a criterion is $\langle 0.9,0.6\rangle$, then $q$ is assigned 3 since $0.9^{2}+0.6^{2}>1$ and $0.9^{3}+0.6^{3}<1$. All of the values in $\mathbf{M}_{\mathrm{MM}}$ and $\mathbf{M}_{\mathrm{GMM}}$ can satisfy $\mu+\nu \leq 1$, and thus, the value of $q$ in the experiment can be assigned from 1 .

5.2.3. Experiment 3. It was carried out to show the influence of the assignment of different values to the parameter $\lambda$ on the aggregation results. In this experiment, $\mathbf{M}_{\mathrm{MM}}$ and $\mathbf{w}$ and $\mathbf{M}_{\mathrm{GMM}}$ and $\mathbf{w}$ in the example were, respectively, used as the input of the qROFWAHMM operator $\left(\Delta=\left(\delta_{1}, \delta_{2}, \delta_{3}\right.\right.$, $\left.\delta_{4}\right)=(1,2,3,4), q=1$, and $\lambda$ whose value ranges from 0.0001 to 20) in equation (66) and the qROFWAHGMM operator $\left(\Delta=\left(\delta_{1}, \delta_{2}, \delta_{3}, \delta_{4}\right)=(1,2,3,4), q=1\right.$, and $\lambda$ whose value ranges from 0.0001 to 20 ) in equation (67). The results of the experiment are the calculated scores of the collective overall preferences of the five companies, which are depicted in Figure 2. It can be seen from the figure that the scores of the five companies computed by qROFWAHMM gradually increase, the scores computed by qROFWAHGMM gradually decrease, and the rankings generated by the two operators remain the same, as the value of $\lambda$ gradually increases. Thus, the parameter $\lambda$ can be seen as an optimistic factor for the qROFWAHMM operator, while a pessimistic factor for the qROFWAHGMM operator. Generally, if the attitude is neutral, a small $\lambda(1,2$, or 3$)$ is recommended. If the attitude is optimistic enough, a bigger (smaller) $\lambda$ can be assigned when qROFWAHMM (qROFWAHGMM) is used. Otherwise, a smaller (bigger) $\lambda$ is recommended.

5.2.4. Experiment 4. It was carried out to show the influence of the assignment of different values to the parameters $\delta_{1}, \delta_{2}$, $\delta_{3}$, and $\delta_{4}$ on the aggregation results. In this experiment, $\mathbf{M}_{\mathrm{MM}}$ and $\mathbf{w}$ and $\mathbf{M}_{\mathrm{GMM}}$ and $\mathbf{w}$ in the example were, respectively, used as the input of the qROFWAHMM operator $\left(\Delta=\left(\delta_{1}, \delta_{2}, \delta_{3}, \delta_{4}\right)=(1,0,0,0) ;(1,2,0,0) ;(1,2,3,0) ;(1,2,3\right.$, 4), $q=1$, and $\lambda=3$ ) in equation (66) and the qROFWAHGMM operator $\left(\Delta=\left(\delta_{1}, \delta_{2}, \delta_{3}, \delta_{4}\right)=(1,0,0,0) ;(1,2,0\right.$, $0)$; $(1,2,3,0) ;(1,2,3,4), q=1$, and $\lambda=3)$ in equation (67). The results of the experiment are the calculated scores of the collective overall preferences of the five companies and the generated rankings of the five companies, which are listed in Table 2. As can be seen from the table, the rankings of the five companies generated by the two operators remain the same, in the cases where (1) all of the four criteria are independent of each other, (2) there are interrelationships between any two criteria, (3) there are interrelationships between any three criteria, and (4) there are interrelationships between all of the four criteria. From such results, it is difficult to see the differences of how the four cases affect the aggregation results. Even so, this does not mean that such differences do not exist, since the numerical example is a very simple example that includes only four criteria. For complex examples with more criteria, the differences may be more obvious. 


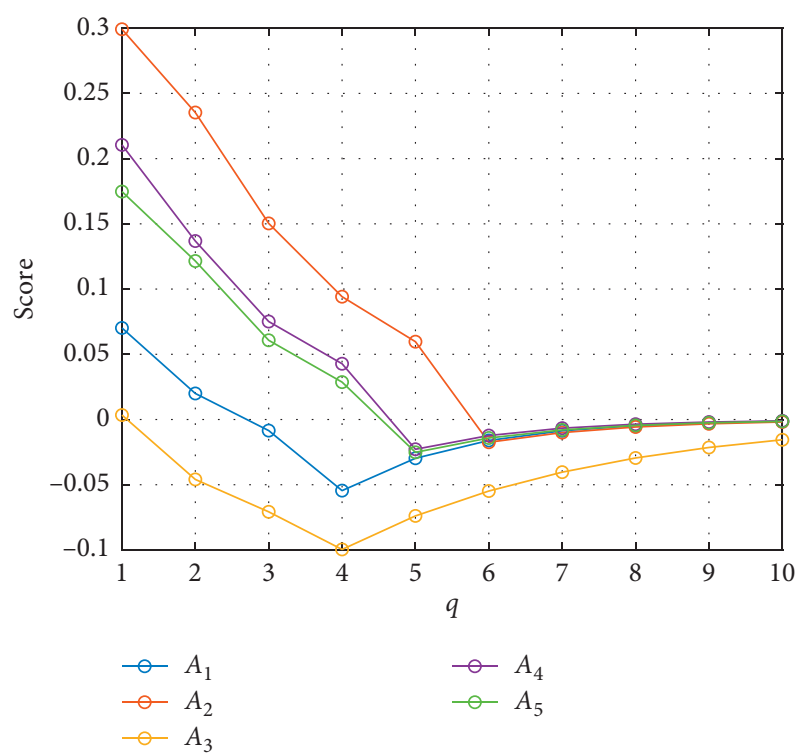

(a)

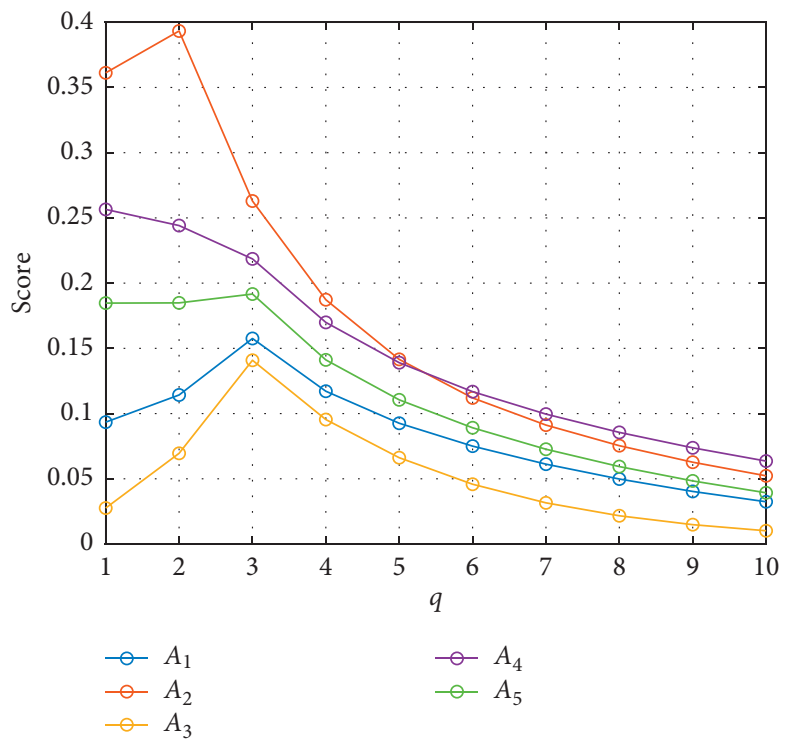

(b)

FIgURE 1: The results of Experiment 2. Scores of the five companies calculated by (a) qROFWAHMM and (b) qROFWAHGMM.

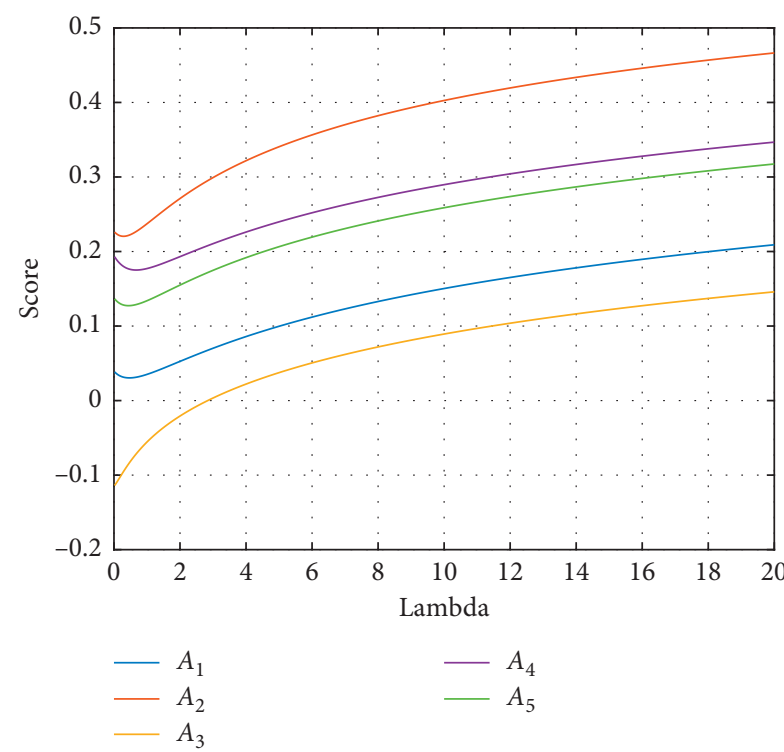

(a)

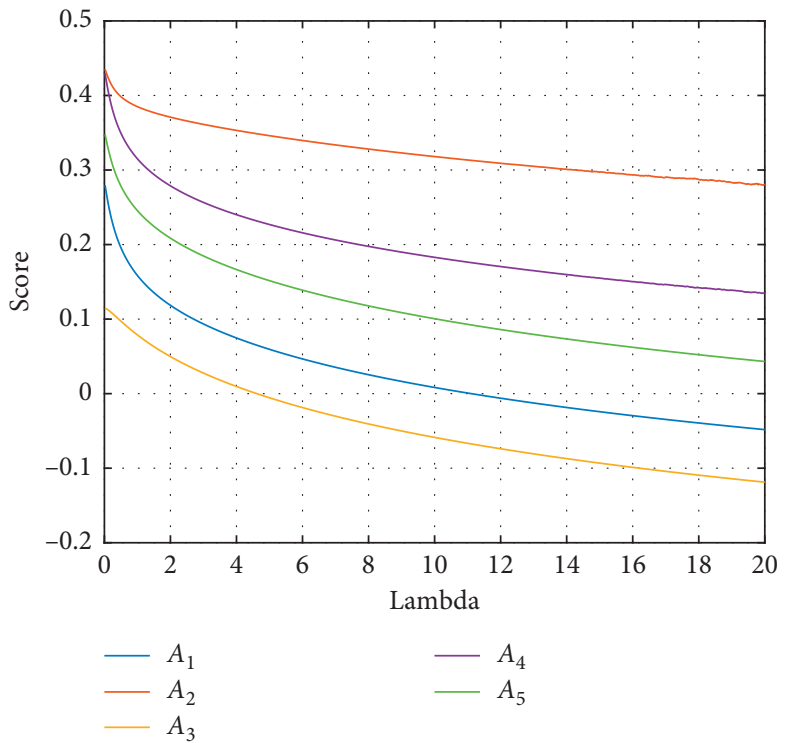

(b)

Figure 2: The results of Experiment 3. Scores of the five companies calculated by (a) qROFWAHMM and (b) qROFWAHGMM.

\subsection{Comparisons}

5.3.1. Comparisons with Muirhead Aggregation Operators of IFNs and PFNs. It is of necessity to make comparisons with the MCGDM methods based on Muirhead aggregation operators of IFNs and PFNs because these methods are very relevant to the proposed MCGDM method. At present, a number of Muirhead aggregation operators of IFNs and PFNs have been presented. Representative examples are the intuitionistic fuzzy MM (IFMM) and intuitionistic fuzzy GMM (IFGMM) operators presented by Liu and Li [55] and the Pythagorean fuzzy MM (PFMM) and Pythagorean fuzzy
GMM (PFGMM) operators presented by Zhu and Li [56]. In this subsection, the following qualitative and quantitative comparisons between the methods based on these operators and the proposed method were carried out.

(1) Qualitative Comparison. In general, a qualitative comparison among different MCGDM methods can be made by comparing their characteristics. For the four existing methods and the proposed method, the application range from the perspective of the values of criteria, the generality and flexibility in the aggregation of the values of criteria, and the capability to capture the attitudes of decision makers are 
TABLE 2: The results of Experiment 4.

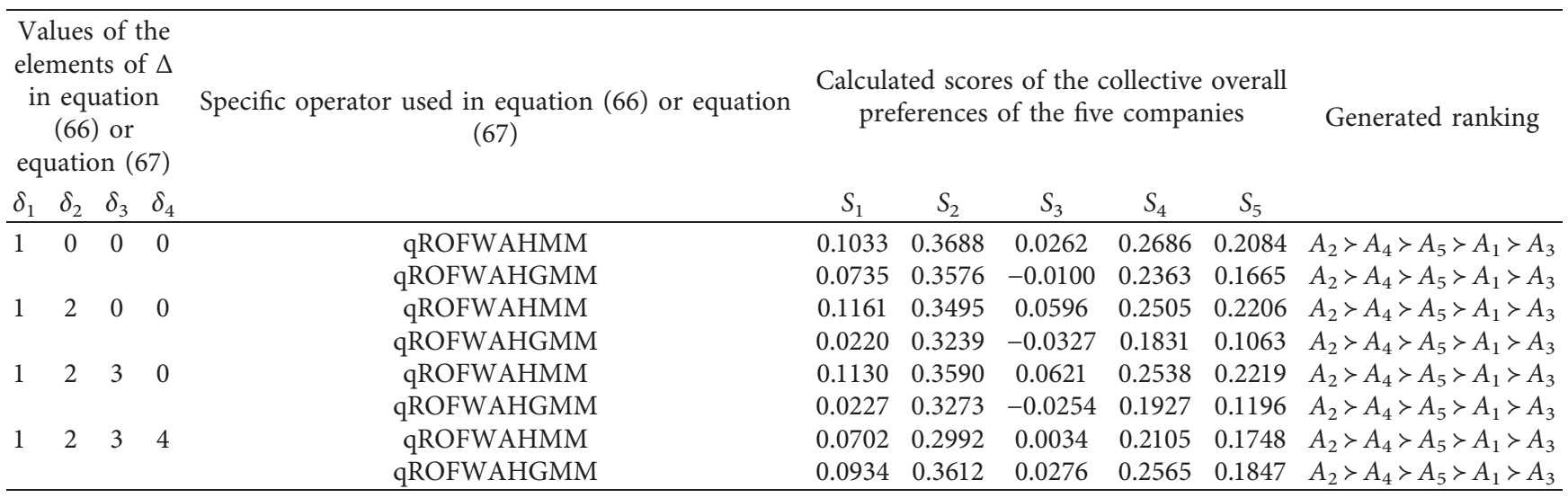

TABLE 3: The results of the qualitative comparison with the MCGDM methods based on Muirhead aggregation operators of IFNs and PFNs.

\begin{tabular}{|c|c|c|c|}
\hline MCGDM method & $\begin{array}{l}\text { Application range from the perspective } \\
\text { of the values of criteria }\end{array}$ & $\begin{array}{l}\text { Generality and flexibility in the } \\
\text { aggregation }\end{array}$ & $\begin{array}{l}\text { Capability to capture the } \\
\text { attitudes }\end{array}$ \\
\hline IFMM [55] & When the values of all criteria are in IFNs & Limited & No \\
\hline IFGMM [55] & When the values of all criteria are in IFNs & Limited & No \\
\hline PFMM [56] & When the values of all criteria are in IFNs or PFNs & Limited & No \\
\hline PFGMM [56] & When the values of all criteria are in IFNs or PFNs & Limited & No \\
\hline qROFWAMM & $\begin{array}{l}\text { When the values of all criteria are in IFNs or PFNs or } \\
\text { qROFNs }\end{array}$ & Satisfying & Yes \\
\hline qROFWAGMM & $\begin{array}{l}\text { When the values of all criteria are in IFNs or PFNs or } \\
\text { qROFNs }\end{array}$ & Satisfying & Yes \\
\hline
\end{tabular}

selected as the comparison characteristics. The results of the comparison are shown in Table 3. The details of the comparison are explained as follows:

(1) Application range from the perspective of the values of criteria: the methods based on the IFMM and IFGMM operators are applicable when the values of all criteria are in IFNs. The methods based on the PFMM and PFGMM operators can be applied when the values of all criteria are in PFNs, which include IFNs. The proposed method is applicable when the values of all criteria are in qROFNs, which include both IFNs and PFNs. The relationships among IFNs, PFNs, and qROFNs are depicted in Figure 3. From the figure, it can be seen intuitively that the proposed method has the widest application range among all comparison methods. In other words, the methods based on the IFMM, IFGMM, PFMM, and PFGMM operators are just the special cases of the proposed method (to be more specific, qROFWAMM (qROFWAGMM) will reduce to IFMM (IFGMM) if $f(t)=-\operatorname{In} t^{q}$ and $q=1$ and will reduce to PFMM (PFGMM) if $f(t)=-\operatorname{In} t^{q}$ and $q=2$ ).

(2) Generality and flexibility in the aggregation: the generality and flexibility of the proposed method are desirable because the aggregations in it are based on the operations of any family of ATTs. The

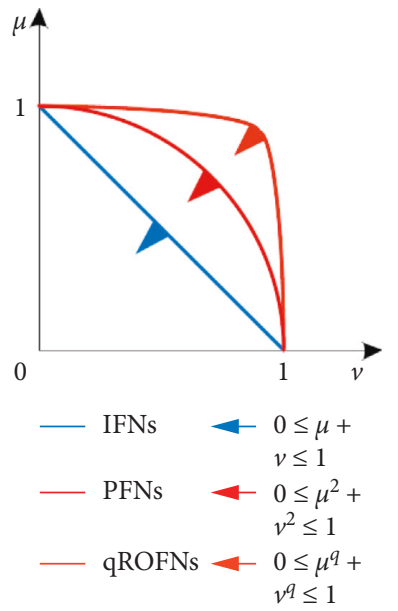

Figure 3: The relationships among IFNs, PFNs, and qROFNs.

aggregations in the remaining methods are based on the fixed Algebraic T-norm and T-conorm operation. These methods relatively have limited generality and flexibility.

(3) Capability to capture the attitudes: the proposed method has this capability because the aggregations in it are based on the operations of any family of ATTs and some families of ATTs (e.g., Hamacher 
T-norm and T-conorm, and Frank T-norm and T-conorm) can provide flexible parameters to describe the attitudes of decision makers. The remaining methods do not have the capability since the aggregations in them are based on the fixed Algebraic T-norm and T-conorm operation.

As can be summarized from the qualitative comparison, the proposed method can provide more flexible application range and more desirable generality and flexibility in the aggregation of the values of criteria and the capturing of the attitudes of decision makers over the methods based on Muirhead aggregation operators of IFNs and PFNs.

(2) Quantitative Comparison. Generally, a quantitative comparison among different MCGDM methods can be carried out using the same numerical example. The numerical example in Subsection 5.1 is used to quantitatively compare the MCGDM methods based on the IFMM, IFGMM, PFMM, and PFGMM operators and the proposed MCGDM method. The results of this comparison are the calculated scores of the collective overall preferences of the five companies and the generated rankings of the five companies, which are listed in Table 4 . As can be seen from the table, all of the comparison methods can be used to solve the MCGDM problem in the example and the rankings generated by them are exactly the same. This is because the values of all criteria in the example are in IFNs (i.e., the values of all criteria can satisfy $\mu+\nu \leq 1$ ). From these results, it is difficult to see the superiority of the proposed method over other comparison methods.

To show the superiority, suppose the value of $\Xi_{1,4,1}$ in $\mathbf{M}_{1}$ is changed to $\langle 0.8,0.7\rangle$ (such value is entirely possible in practical MCGDM problems) and the same methods are applied to solve the changed MCGDM problem. The results are also listed in Table 4. It can be seen from the table that only the proposed method is applicable and can generate results. The reason is that $\langle 0.8,0.7\rangle$ cannot satisfy $\mu+\nu \leq 1$ and $\mu^{2}+\nu^{2} \leq 1$, but can satisfy $\mu^{q}+\nu^{q} \leq 1(q=3,4,5, \ldots)$. This means that the application range of the proposed method from the perspective of the values of criteria can be adjusted by setting an appropriate value to the flexible parameter $q$. Thus, it can be concluded that the proposed method can provide more flexible application range over the methods based on Muirhead aggregation operators of IFNs and PFNs.

5.3.2. Comparisons with Aggregation Operators of qROFNs. It is also of necessity to make comparisons with the MCGDM methods based on aggregation operators of qROFNs, since both these methods and the proposed MCGDM method are for qROFNs. As mentioned in the introduction, more than twenty different aggregation operators of qROFNs have been presented so far. The representative aggregation operators are the WE [38], WP [39], WA and WG [40], WBM and WGBM [41], WABM [42], WEBM [43], WPBM and WPGBM [44], WHM and WGHM [45], WHM* and WPHM [46], WMSM and WGMSM [47],
WPMSM [48], WPPMSM [49], and WMM and WGMM [50] operators. In this subsection, the following qualitative and quantitative comparisons between the methods based on these operators and the proposed method were carried out.

(1) Qualitative Comparison. For the twenty existing methods and the proposed method, the generality and flexibility in the aggregation of qROFNs, the capability to deal with the cases where all criteria are independent of each other, there are interrelationships between any two criteria, and there are interrelationships among any multiple (more than two) criteria, and the capability to capture the attitudes of decision makers are selected as the comparison characteristics. The results of the comparison are shown in Table 5. The details of the comparison are explained as follows:

(1) Generality and flexibility in the aggregation: for the method based on the WP operator, any one of the twenty WP operators that have different operations can be used in the aggregation. Thus, its generality and flexibility can be seen as moderate. The generality and flexibility of the methods based on the WABM operator and the proposed method are desirable because the aggregations in them are based on the operations of any family of ATTs. The aggregations in the remaining methods are based on based on the fixed Algebraic T-norm and T-conorm operation. These methods relatively have limited generality and flexibility.

(2) When all criteria are independent of each other: it is no doubt that all methods can deal with this case.

(3) When there are interrelationships between any two criteria: the methods based on the WE, WP, WA, and WG operators are only suitable for the independent case. Except these methods, all methods can handle this situation.

(4) When there are interrelationships among any multiple criteria: among all methods, the methods based on the WMSM, WGMSM, WPMSM, WPPMSM, WMM, and WGMM operators can process this case due to the use of the MSM or MM operator in them.

(5) Capability to capture the attitudes: the methods based on the WABM operator and the proposed method have this capability because the aggregations in them are based on the operations of any family of ATTs and some families of ATTs (e.g., Hamacher T-norm and T-conorm, and Frank T-norm and $\mathrm{T}$-conorm) can provide flexible parameters to describe the attitudes of decision makers. The remaining methods do not have the capability since aggregations in them are based on the fixed Algebraic T-norm and T-conorm operation.

As can be summarized from the qualitative comparison, the proposed method has desirable generality and flexibility at both aggregating the values of criteria and capturing the interrelationships of criteria and the attitudes of decision makers. 
TABLE 4: The results of the quantitative comparison with the MCGDM methods based on Muirhead aggregation operators of IFNs and PFNs. Notes: when comparing the proposed method, the qROFWAHMM and qROFWAHGMM operators were selected. When adapting the groups of IFMMs, IFGMMs, PFMMs, and PFGMMs, $\Delta=\left(\delta_{1}, \delta_{2}, \delta_{3}\right)=(1,0,0)$ for IFMM and PFMM in equation (64) and IFGMM and PFGMM in equation (65), and $\Delta=\left(\delta_{1}, \delta_{2}, \delta_{3}\right)=(1,2,3,4)$ for IFMM and PFMM in equation (66) and IFGMM and PFGMM in equation (67). When adapting the groups of qROFWAHMMs and qROFWAHGMMs, $q=3, \lambda=3$, and $\Delta=\left(\delta_{1}, \delta_{2}, \delta_{3}\right)=(1,0,0)$ for qROFWAHMM in equation (64) and qROFWAHGMM in equation (65), and $q=1, \lambda=3$, and $\Delta=\left(\delta_{1}, \delta_{2}, \delta_{3}\right)=(1,2,3,4)$ for qROFWAHMM in equation (66) and qROFWAHGMM in equation (67).

\begin{tabular}{|c|c|c|c|c|c|c|c|c|}
\hline \multirow{2}{*}{$\begin{array}{l}\text { Value of } \\
\Xi_{1,4,1}\end{array}$} & \multirow{2}{*}{$\begin{array}{c}\text { Specific operator used in } \\
\text { equation }(64) \text { or equation } \\
(65)\end{array}$} & \multirow{2}{*}{$\begin{array}{c}\text { Specific operator used in } \\
\text { equation }(66) \text { or equation } \\
(67)\end{array}$} & \multicolumn{5}{|c|}{$\begin{array}{l}\text { Calculated scores of the collective overall } \\
\text { preferences of the five companies }\end{array}$} & \multirow[t]{2}{*}{ Generated ranking } \\
\hline & & & $S_{1}$ & $S_{2}$ & $S_{3}$ & $S_{4}$ & $S_{5}$ & \\
\hline$<0.8,0.2>$ & IFMM [55] & IFMM [55] & 0.0431 & 0.2400 & -0.0460 & 0.1834 & 0.1430 & $A_{2}>A_{4}>A_{5}>A_{1}>A_{3}$ \\
\hline$<0.8,0.2>$ & IFGMM [55] & IFGMM [55] & 0.1508 & 0.3815 & 0.0698 & 0.3077 & 0.2321 & $A_{2}>A_{4}>A_{5}>A_{1}>A_{3}$ \\
\hline$<0.8,0.2>$ & PFMM [56] & PFMM [56] & 0.0353 & 0.2196 & -0.0545 & 0.1525 & 0.1277 & $A_{2}>A_{4}>A_{5}>A_{1}>A_{3}$ \\
\hline$<0.8,0.2>$ & PFGMM [56] & PFGMM [56] & 0.1410 & 0.3447 & 0.0696 & 0.2830 & 0.2038 & $A_{2}>A_{4}>A_{5}>A_{1}>A_{3}$ \\
\hline$<0.8,0.2>$ & qROFWAMM & qROFWAMM & 0.0863 & 0.3024 & 0.0303 & 0.2186 & 0.1871 & $A_{2}>A_{4}>A_{5}>A_{1}>A_{3}$ \\
\hline$<0.8,0.2>$ & qROFWAGMM & qROFWAGMM & 0.0713 & 0.3519 & 0.0052 & 0.2414 & 0.1458 & $A_{2}>A_{4}>A_{5}>A_{1}>A_{3}$ \\
\hline$<0.8,0.7>$ & IFMM [55] & IFMM [55] & \multicolumn{5}{|c|}{ Cannot be calculated } & Cannot generate \\
\hline$<0.8,0.7>$ & IFGMM [55] & IFGMM [55] & \multicolumn{5}{|c|}{ Cannot be calculated } & Cannot generate \\
\hline$<0.8,0.7>$ & PFMM [56] & PFMM [56] & \multicolumn{5}{|c|}{ Cannot be calculated } & Cannot generate \\
\hline$<0.8,0.7>$ & PFGMM [56] & PFGMM [56] & \multicolumn{5}{|c|}{ Cannot be calculated } & Cannot generate \\
\hline$<0.8,0.7>$ & qROFWAMM & qROFWAMM & 0.0863 & 0.3024 & 0.0303 & 0.1830 & 0.1871 & $A_{2}>A_{5}>A_{4}>A_{1}>A_{3}$ \\
\hline$<0.8,0.7>$ & qROFWAGMM & qROFWAGMM & 0.0713 & 0.3519 & 0.0052 & 0.1976 & 0.1458 & $A_{2}>A_{4}>A_{5}>A_{1}>A_{3}$ \\
\hline
\end{tabular}

TABLE 5: The results of the qualitative comparison with the MCGDM methods based on aggregation operators of qROFNs.

\begin{tabular}{|c|c|c|c|c|c|}
\hline \multirow{2}{*}{ MCGDM method } & \multirow{2}{*}{$\begin{array}{l}\text { Generality and flexibility in the } \\
\text { aggregation }\end{array}$} & \multicolumn{3}{|c|}{$\begin{array}{l}\text { Capability to deal with the interrelationships of } \\
\text { criteria }\end{array}$} & \multirow{2}{*}{$\begin{array}{l}\text { Capability to capture the } \\
\text { attitudes }\end{array}$} \\
\hline & & Independent & $\begin{array}{l}\text { Any two } \\
\text { criteria }\end{array}$ & $\begin{array}{l}\text { Any multiple } \\
\text { criteria }\end{array}$ & \\
\hline qROFWE [38] & Limited & Yes & No & No & No \\
\hline qROFWP [39] & Moderate & Yes & No & No & No \\
\hline qROFWA [40] & Limited & Yes & No & No & No \\
\hline qROFWG [40] & Limited & Yes & No & No & No \\
\hline qROFWBM [41] & Limited & Yes & Yes & No & No \\
\hline qROFWGBM [41] & Limited & Yes & Yes & No & No \\
\hline qROFWABM [42] & Satisfying & Yes & Yes & No & Yes \\
\hline qROFWEBM [43] & Limited & Yes & Yes & No & No \\
\hline qROFWPBM [44] & Limited & Yes & Yes & No & No \\
\hline $\begin{array}{l}\text { qROFWPGBM } \\
\text { [44] }\end{array}$ & Limited & Yes & Yes & No & No \\
\hline qROFWHM [45] & Limited & Yes & Yes & No & No \\
\hline qROFWGHM [45] & Limited & Yes & Yes & No & No \\
\hline qROFWHM* ${ }^{*}$ [46] & Limited & Yes & Yes & No & No \\
\hline qROFWPHM [46] & Limited & Yes & Yes & No & No \\
\hline qROFWMSM [47] & Limited & Yes & Yes & Yes & No \\
\hline $\begin{array}{l}\text { qROFWGMSM } \\
\text { [47] }\end{array}$ & Limited & Yes & Yes & Yes & No \\
\hline $\begin{array}{l}\text { qROFWPMSM } \\
{[48]}\end{array}$ & Limited & Yes & Yes & Yes & No \\
\hline $\begin{array}{l}\text { qROFWPPMSM } \\
\text { [49] }\end{array}$ & Limited & Yes & Yes & Yes & No \\
\hline qROFWMM [50] & Limited & Yes & Yes & Yes & No \\
\hline $\begin{array}{l}\text { qROFWGMM } \\
{[50]}\end{array}$ & Limited & Yes & Yes & Yes & No \\
\hline qROFWAMM & Satisfying & Yes & Yes & Yes & Yes \\
\hline qROFWAGMM & Satisfying & Yes & Yes & Yes & Yes \\
\hline
\end{tabular}

(2) Quantitative Comparison. The numerical example in Subsection 5.1 is used to quantitatively compare the MCGDM methods based on the WA, WG, WBM, WGBM,
WABM, WHM, WGHM, WHM*, WMSM, WGMSM, WMM, and WGMM operators (please note that the methods based on the WE, WP, WEBM, WPBM, WPGBM, 
TABLE 6: The results of the quantitative comparison with the MCGDM methods based on aggregation operators of qROFNs. Notes: for easy comparison, all the methods used the same score function (see Definition 2). When comparing the method based on WABM, the most generalized q-rung orthopair fuzzy weighted Archimedean Hamacher Bonferroni mean (WAHBM) operator was selected. When comparing the proposed method, the most generalized WAHMM and WAHGMM operators were selected. When adapting the fourteen groups of operators, $q=1$ for all groups of operators; $\lambda=3$ for the groups of WAHBMs, WAHMMs, and WAHGMMs. $s=1(a=1)$ and $t=0(b=0)$ for WBM and WAHBM (WHM and WHM*) in equation (64) and WGBM (WGHM) in equation (65); $s=1(a=1)$ and $t=2(b=2)$ for WBM and WAHBM (WHM and WHM WH $^{*}$ in equation (66) and WGBM (WGHM) in equation $(67) ; k=1\left(\Delta=\left(\delta_{1}, \delta_{2}, \delta_{3}\right)=(1,0,0)\right)$ for WMSM (WMM and WAHMM) in equation (64) and WGMSM (WGMM and WAHGMM) in equation $(65)$; and $k=4\left(\Delta=\left(\delta_{1}, \delta_{2}, \delta_{3}\right)=(1\right.$, $2,3,4)$ ) for WMSM (WMM and WAHMM) in equation (66) and WGMSM (WGMM and WAHGMM) in equation (67).

\begin{tabular}{|c|c|c|c|c|c|c|c|}
\hline \multirow{2}{*}{$\begin{array}{l}\text { Specific operator used in } \\
\text { equation (64) or equation (65) }\end{array}$} & \multirow{2}{*}{$\begin{array}{l}\text { Specific operator used in } \\
\text { equation }(66) \text { or equation }(67)\end{array}$} & \multicolumn{5}{|c|}{$\begin{array}{l}\text { Calculated scores of the collective overall } \\
\text { preferences of the five companies }\end{array}$} & \multirow[t]{2}{*}{ Generated ranking } \\
\hline & & $S_{1}$ & $S_{2}$ & $S_{3}$ & $S_{4}$ & $S_{5}$ & \\
\hline qROFWA [40] & qROFWA [40] & 0.1144 & 0.3724 & 0.0417 & 0.2810 & 0.2244 & $A_{2}>A_{4}>A_{5}>A_{1}>A_{3}$ \\
\hline qROFWG [40] & qROFWG [40] & 0.0592 & 0.3523 & -0.0261 & 0.2212 & 0.1455 & $A_{2}>A_{4}>A_{5}>A_{1}>A_{3}$ \\
\hline qROFWBM [41] & qROFWBM [41] & -0.8638 & & -0.8753 & -0.8295 & -0.8 & $A_{2}>A_{4}>A_{5}>A_{1}>A_{3}$ \\
\hline qROFWGBM [41] & qROFWGBM [41] & 0.8917 & 0.9342 & 0.87 & 0.9 & 0.9058 & $A_{2}>A_{4}>A_{5}>A_{1}>A_{3}$ \\
\hline qROFWABM [42] & qROFWABM [42] & 0.1161 & 0.3495 & 0.0596 & 0.2505 & 0.2206 & $A_{2}>A_{4}>A_{5}>A_{1}>A_{3}$ \\
\hline qROFWHM [45] & qROFWHM [45] & -0.3015 & -0.0385 & -0.2834 & -0.0923 & -0.2129 & $A_{2}>A_{4}>A_{5}>A_{3}>A_{1}$ \\
\hline & & & & & & 0.4046 & $A_{2}>A_{4}>A_{5}>A_{1}>A_{3}$ \\
\hline $\mathrm{qRO}$ & $\mathrm{NHM}^{*}[46]$ & -0.8509 & -0.7779 & -0.8495 & -0.7931 & -0.8298 & $A_{2}>A_{4}>A_{5}>A_{3}>A_{1}$ \\
\hline qROFWMSM [47] & qROFWMSM [47] & 0.9035 & 0.9383 & 0.8908 & 0.9200 & 0.9159 & $A_{2}>A_{4}>A_{5}>A_{1}>A_{3}$ \\
\hline qROFWGMSM [47] & qROFWGMSM [47] & -0.8696 & -0.8179 & -0.8839 & -0.8407 & -0.8590 & $A_{2}>A_{4}>A_{5}>A_{1}>A_{3}$ \\
\hline qROFWMM [50] & qROFWMM [50] & 0.0431 & 0.2400 & -0.0460 & 0.1834 & 0.1430 & $A_{2}>A_{4}>A_{5}>A_{1}>A_{3}$ \\
\hline qROFWGMM [50] & qROFWGMM [50] & 0.1508 & 0.3815 & 0.0698 & 0.3077 & 0.2321 & $A_{2}>A_{4}>A_{5}>A_{1}>A_{3}$ \\
\hline qROFWAMM & qROFWAMM & 0.0702 & 0.2992 & 0.0034 & 0.2105 & 0.1748 & $A_{2}>A_{4}>A_{5}>A_{1}>A_{3}$ \\
\hline qROFWAGMM & qROFWAGMM & 0.0934 & 0.3612 & 0.0276 & 0.2565 & 0.1847 & $A_{2}>A_{4}>A_{5}>A_{1}>A_{3}$ \\
\hline
\end{tabular}

WPHM, WPMSM, and WPPMSM operators were not included in the quantitative comparison because of the following: the WE operator was presented to deal with the case in which the values of criteria are expressed by a fuzzy number in Zadeh's FS and the values of weights are described by qROFNs; the WP operator was presented to control the uncertainty of the evaluation results of some experts; the WEBM operator was presented to handle the heterogeneous relationship among criteria and unknown criterion weights; the WPBM, WPGBM, and WPHM operators were presented to handle heterogeneous interrelationships of criteria; the WPMSM operator was presented to reduce the influence of biased evaluation values on the aggregation result; the WPPMSM operator was presented to deal with the situation with heterogeneous interrelationships of criteria and some biased evaluation values; and the proposed method does not consider these characteristics) and the proposed MCGDM method. The results of this comparison are the calculated scores of the collective overall preferences of the five companies and the generated rankings of the five companies, which are listed in Table 6.

As can be seen from Table 6, the rankings of the proposed method are exactly the same as the rankings of the methods based on the WA, WG, WBM, WGBM, WABM, WGHM, WMSM, WGMSM, WMM, and WGMM operators, and it just has differences at the fourth and fifth places with the rankings of the methods based on the WHM and $\mathrm{WHM}^{*}$ operators. These results indicate its feasibility and effectiveness in solving the MCGDM problems based on
qROFNs. However, because of such results, it is difficult to see the differences of the comparison methods intuitively.

To show the differences more intuitively, the elements $\Xi_{k, 2,4}(k=1,2,3)$ in $\mathbf{M}_{\mathbf{N}, k}$ were constantly adjusted according to Table 7. It is easy to guess that such adjustments will affect the ranking of $A_{2}$, which may probably be changed from the best company to the worst one because the membership degree becomes smaller and smaller and the nonmembership degree becomes larger and larger. To confirm this conjecture, Figures 4-8, respectively, depict the change of the places of $A_{2}$ in the rankings generated by the methods based on the group of WAs and the group of WGs, the group of WBMs and the group of WGBMs, the group of WHMs and the group of WGHMs, the group of WMSMs and the group of WGMSMs, and the group of WAMMs and the group of WAGMMs. From these figures, it can be seen that the results generated by all of the ten comparison methods are consistent with the conjecture. This also demonstrates the effectiveness of the proposed method. Further, it can also be seen that the ten methods have different sensitivities to the changes in the input values of criteria, which is reflected in the difference in the backward speed of the ranking of $A_{2}$. Among the methods based on WAs, WBMs, WHMs, WMSMs, and WAMMs, the backward speed of WMSMs is the fastest, while the backward speed of WAs and WHMs is the slowest. Among the methods based on WGs, WGBMs, WGHMs, WGMSMs, and WAGMMs, the methods based on WGs and WGHMs have the fastest backward speed, while the method based on the WAGMMs has the slowest backward speed, and the backward speed of WGMSMs is 
TABLE 7: Seven groups of $\Xi_{k, 2,4}(k=1,2,3)$ in $\mathbf{M}_{\mathbf{N}, k}$.

\begin{tabular}{llll}
\hline Number & $\Xi_{1,2,4}$ & $\Xi_{2,2,4}$ & $\Xi_{3,2,4}$ \\
\hline 0 & $<0.6,0.2>$ & $<0.7,0.3>$ & $<0.7,0.2>$ \\
1 & $<0.5,0.3>$ & $<0.6,0.4>$ & $<0.6,0.3>$ \\
2 & $<0.4,0.4>$ & $<0.5,0.5>$ & $<0.5,0.4>$ \\
3 & $<0.3,0.5>$ & $<0.4,0.6>$ & $<0.4,0.5>$ \\
4 & $<0.2,0.6>$ & $<0.3,0.7>$ & $<0.3,0.6>$ \\
5 & $<0.1,0.7>$ & $<0.2,0.8>$ & $<0.2,0.7>$ \\
6 & $<0.1,0.7>$ & $<0.1,0.9>$ & $<0.1,0.8>$ \\
\hline
\end{tabular}

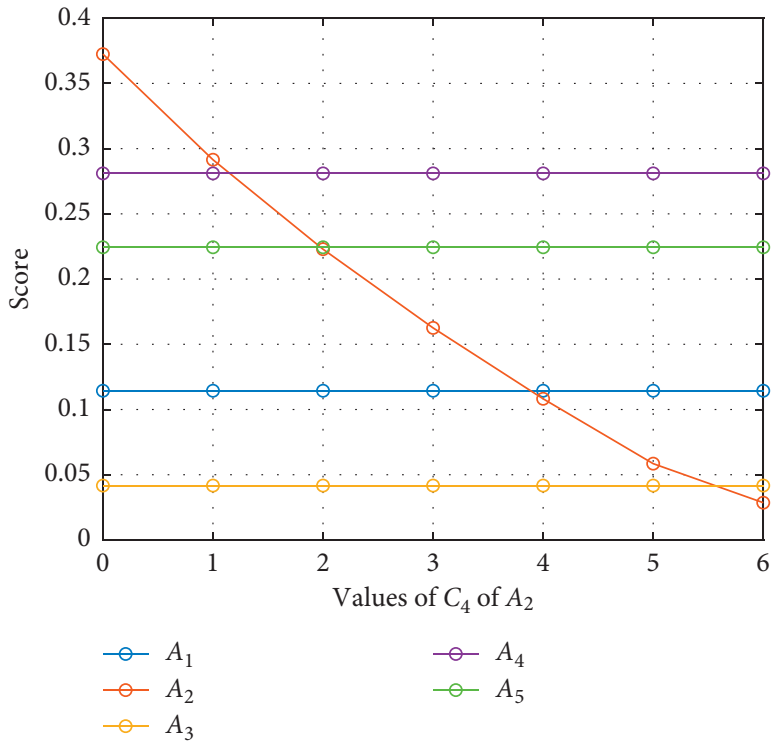

(a)

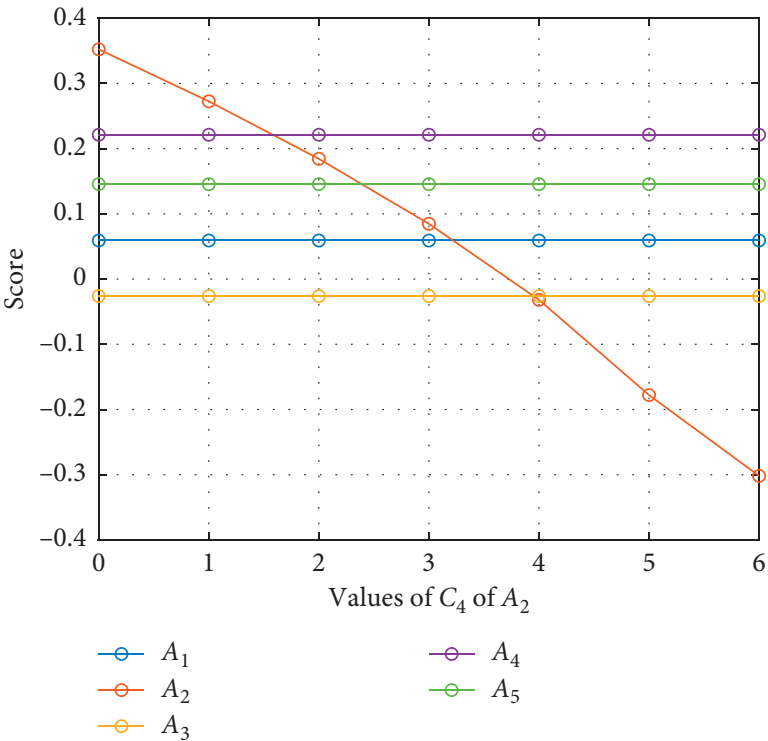

(b)

FIGURE 4: The change of the places of $A_{2}$ in the rankings of the group of WAs [40] and the group of WGs [40]. Scores of the five companies computed by the group of (a) qROFWAs and (b) qROFWGs.

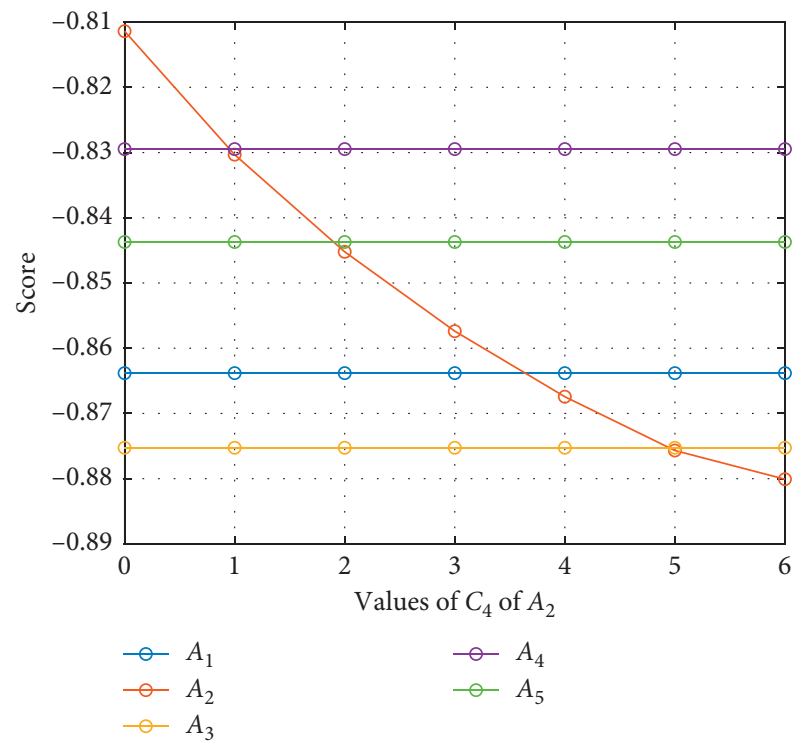

(a)

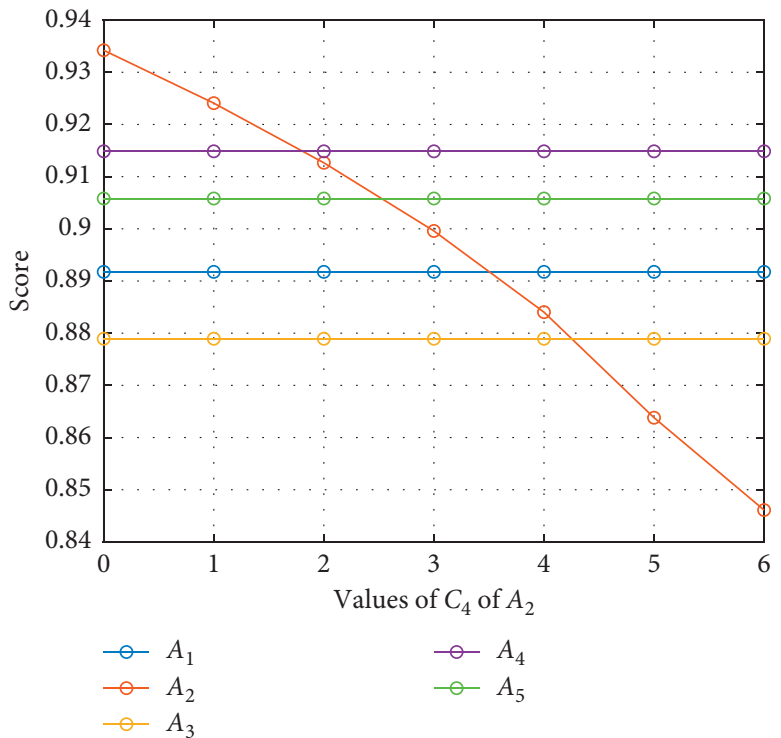

(b)

FIGURE 5: The change of the places of $A_{2}$ in the rankings of the group of WBMs [41] and the group of WGBMs [41]. Scores of the five companies computed by the group of (a) qROFWBMs and (b) qROFWGBMs. 


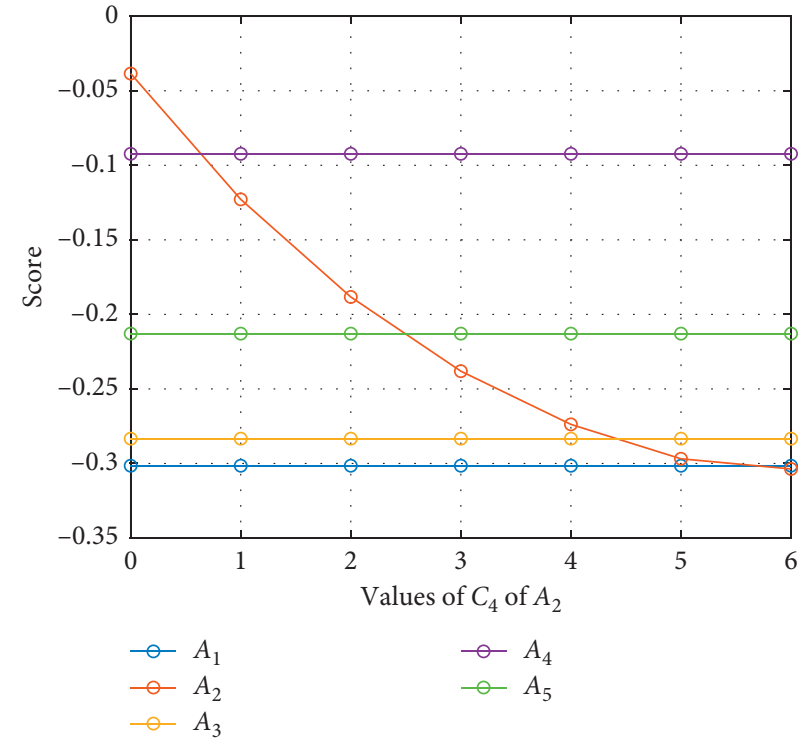

(a)

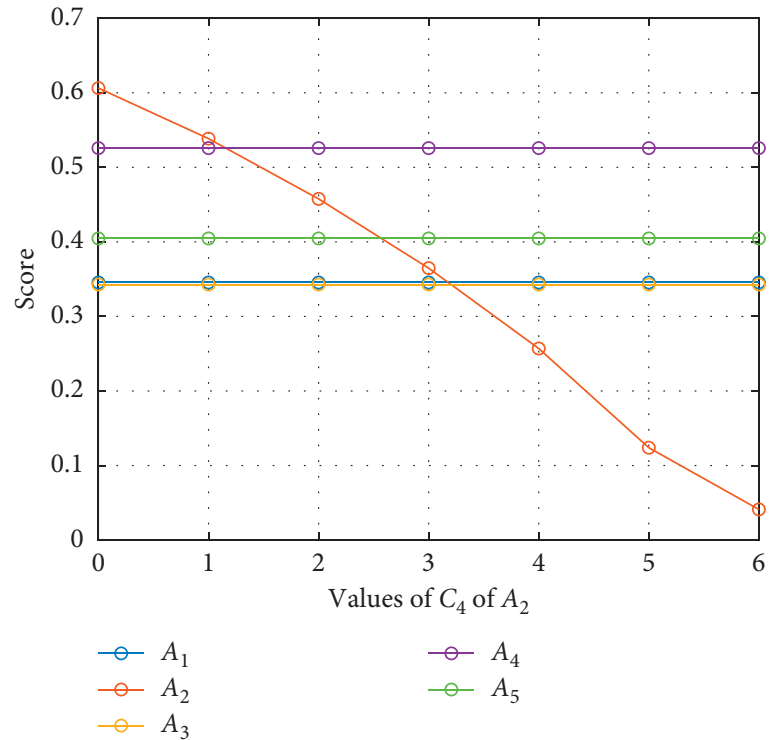

(b)

Figure 6: The change of the places of $A_{2}$ in the rankings of the group of WHMs [45] and the group of WGHMs [45]. Scores of the five companies computed by the group of (a) qROFWHMs and (b) qROFWGHMs.

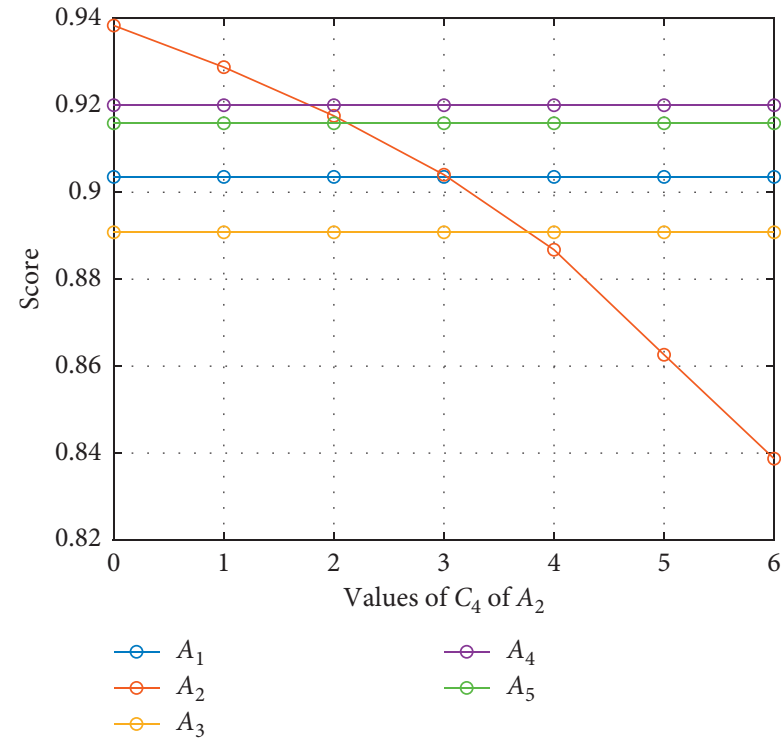

(a)

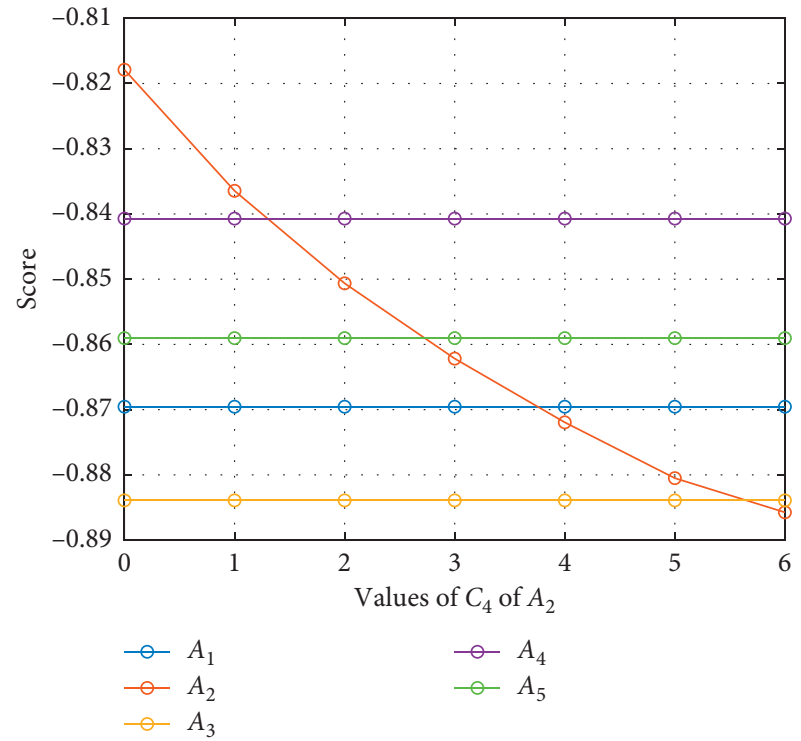

(b)

FIgUre 7: The change of the places of $A_{2}$ in the rankings of the group of WMSMs [47] and the group of WGMSMs [47]. Scores of the five companies computed by the group of (a) qROFWMSMs and (b) qROFWGMSMs.

also slow. From these results, it can be seen that the sensitivities of WAs, WBMs, WHMs, WMSMs, and WAMMs and the sensitivities of their dual forms are opposite, respectively.

Finally, it should be noted that the purpose of the quantitative comparison is not to illustrate that the proposed method is better than the comparison methods, but to verify its feasibility and effectiveness and show the differences of these methods. In general, it is difficult to say that one MCGDM method is better than another one because different methods have different characteristics, which determines their different application scenarios. What users have to do is choose suitable methods for specific application scenarios. 


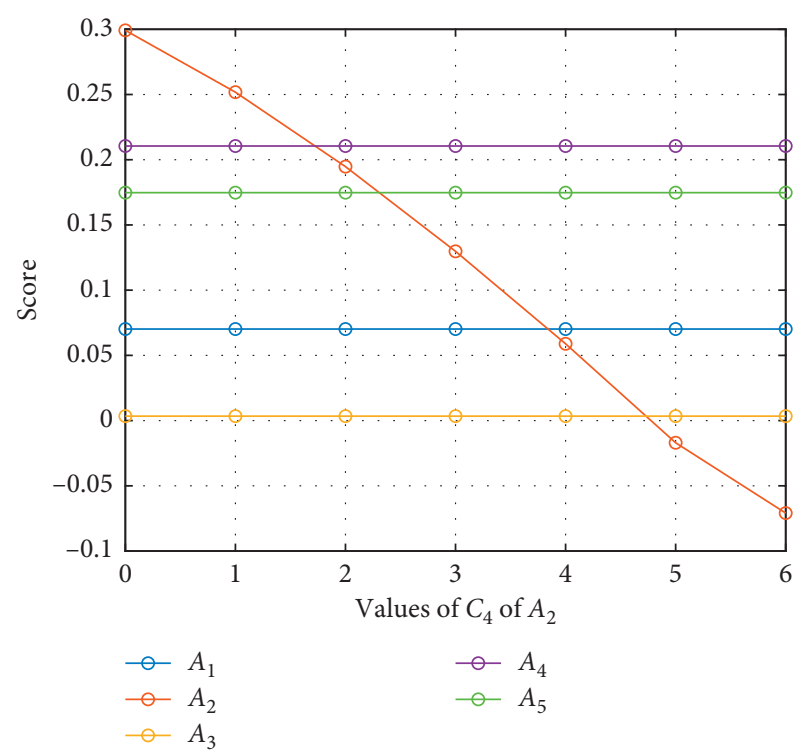

(a)

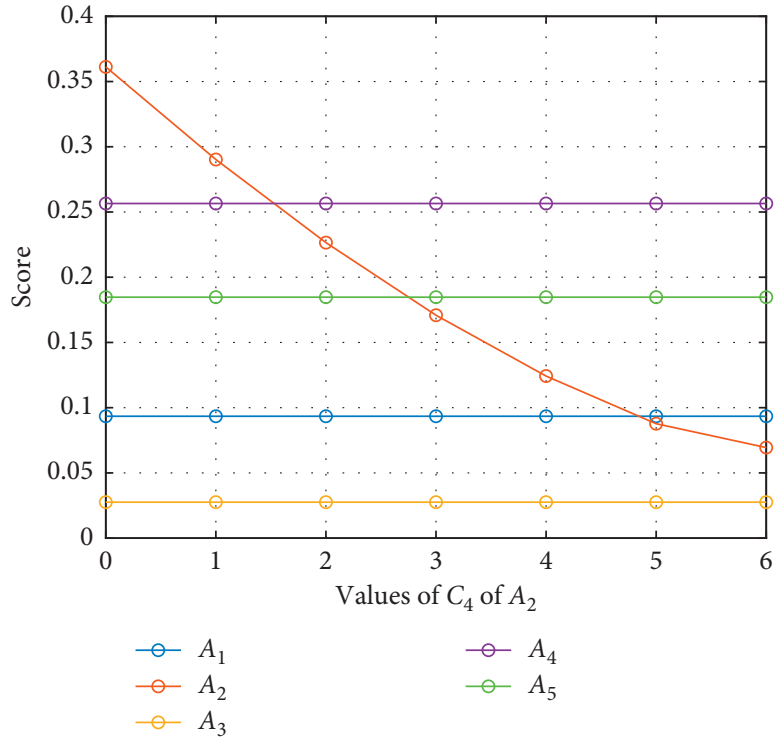

(b)

FIGURE 8: The change of the places of $A_{2}$ in the rankings of the group of WAMMs and the group of WAGMMs. Scores of the five companies computed by the group of (a) qROFWAMMs and (b) qROFWAGMMs.

\section{Conclusion}

In this paper, a qROFAMM operator, a qROFWAMM operator, a qROFAGMM operator, and a qROFWAGMM operator have been presented to aggregate q-rung orthopair fuzzy information for solving the MCGDM problems based on qROFNs. The idempotency, monotonicity, and boundedness of these operators have been proved and their four specific operators have been constructed using the Algebraic, Einstein, Hamacher, and Frank families of ATTs and their additive generators. On the basis of the presented operators, a method for solving the MCGDM problems based on qROFNs has been proposed. The paper has also provided a numerical example to show how the method works, carried out a set of experiments to explore the characteristics of the method, and made qualitative and quantitative comparisons to verify the advantages, feasibility, and effectiveness of the method. The results of the experiments and comparisons suggest that the presented operators and the proposed method are general and flexible at both the aggregation of q-rung orthopair fuzzy information and the capturing of the interrelationships of criteria and the attitudes of decision makers and are feasible and effective for solving the MCGDM problems based on qROFNs.

Future work will focus especially on extending the presented operators from the aspect of dealing with more complex interrelationships of criteria and reducing the influence of biased evaluation values on the aggregation result. The partitioned averaging operator and the power averaging operator will probably be introduced and combined with the presented operators to construct new aggregation operators. Further, the application of the proposed method in solving practical decision-making problems in manufacturing environment, such as determination of additive manufacturing

processes, planning of part build directions in additive manufacturing, and selection of automated inspection systems, will also be studied in the future work.

\section{Acronyms}

ATT:

BM:

FS:

GBM:

GMM:

GMSM:

IFGMM:

IFMM:

IFN:

IFS:

MCDM:

MCGDM:

MM:

MSM:

PFGMM:

PFMM:

PFN:

PFS:

qROFAGMM:

qROFAMM:

qROFN:

qROFS:

qROFAAMM:
Archimedean T-norm and T-conorm Bonferroni mean

Fuzzy set

Geometric Bonferroni mean

Geometric Muirhead mean

Geometric Maclaurin symmetric mean Intuitionistic fuzzy geometric Muirhead mean

Intuitionistic fuzzy Muirhead mean Intuitionistic fuzzy number Intuitionistic fuzzy set Multicriteria decision making Multicriteria group decision making Muirhead mean Maclaurin symmetric mean Pythagorean fuzzy geometric Muirhead mean

Pythagorean fuzzy Muirhead mean Pythagorean fuzzy number Pythagorean fuzzy set q-rung orthopair fuzzy Archimedean geometric Muirhead mean q-rung orthopair fuzzy Archimedean Muirhead mean q-rung orthopair fuzzy number q-rung orthopair fuzzy set q-rung orthopair fuzzy Archimedean Algebraic Muirhead mean 
qROFAEMM:

q-rung orthopair fuzzy Archimedean

Einstein Muirhead mean

qROFAFMM: q-rung orthopair fuzzy Archimedean

Frank Muirhead mean

qROFAHMM: q-rung orthopair fuzzy Archimedean

Hamacher Muirhead mean

qROFWAAMM: q-rung orthopair fuzzy weighted

Archimedean Algebraic Muirhead

mean

qROFAAGMM: q-rung orthopair fuzzy Archimedean

Algebraic geometric Muirhead mean

qROFAEGMM: q-rung orthopair fuzzy Archimedean

Einstein geometric Muirhead mean

qROFAFGMM: q-rung orthopair fuzzy Archimedean frank geometric Muirhead mean

qROFAHGMM: q-rung orthopair fuzzy Archimedean

Hamacher geometric Muirhead mean

qROFWAAGMM: q-rung orthopair fuzzy weighted

Archimedean Algebraic geometric

Muirhead mean

qROFWAEGMM: q-rung orthopair fuzzy weighted

Archimedean Einstein geometric

Muirhead mean

qROFWAFGMM: q-rung orthopair fuzzy weighted

Archimedean Frank geometric

Muirhead mean

qROFWAHGMM: q-rung orthopair fuzzy weighted

Archimedean Hamacher geometric

Muirhead mean

qROFWAEMM: q-rung orthopair fuzzy weighted

Archimedean Einstein Muirhead mean

qROFWAFMM: q-rung orthopair fuzzy weighted

Archimedean Frank Muirhead mean

qROFWAHMM: q-rung orthopair fuzzy weighted

Archimedean Hamacher Muirhead mean

qROFWAMM:

qROFWAGMM: q-rung orthopair fuzzy weighted

Archimedean geometric Muirhead mean

(qROF)WA:

(qROF)WABM:

(q-rung orthopair fuzzy) weighted averaging

(q-rung orthopair fuzzy) weighted

Archimedean Bonferroni mean

(qROF)WBM: (q-rung orthopair fuzzy) weighted

Bonferroni mean

(qROF)WE: (q-rung orthopair fuzzy) weighted exponential

(qROF)WEBM: (q-rung orthopair fuzzy) weighted extended Bonferroni mean

(qROF)WG: (q-rung orthopair fuzzy) weighted geometric

(qROF)WGBM: (q-rung orthopair fuzzy) weighted geometric Bonferroni mean

(qROF)WGHM: (q-rung orthopair fuzzy) weighted geometric Heronian mean
(qROF)WGMM: (q-rung orthopair fuzzy) weighted geometric Muirhead mean

(qROF)WGMSM: (q-rung orthopair fuzzy) weighted geometric Maclaurin symmetric mean

(qROF)WHM: (q-rung orthopair fuzzy) weighted Heronian mean

(qROF)WMM: (q-rung orthopair fuzzy) weighted Muirhead mean

(qROF)WMSM: (q-rung orthopair fuzzy) weighted Maclaurin symmetric mean

(qROF)WP: (q-rung orthopair fuzzy) weighted point

(qROF)WPBM: (q-rung orthopair fuzzy) weighted partitioned Bonferroni mean

(qROF)WPGBM: (q-rung orthopair fuzzy) weighted partitioned geometric Bonferroni mean

(qROF)WPHM: (q-rung orthopair fuzzy) weighted partitioned Heronian mean

(qROF)WPMSM: (q-rung orthopair fuzzy) weighted power Maclaurin symmetric mean

(qROF) (q-rung orthopair fuzzy) weighted

WPPMSM: $\quad$ power partitioned Maclaurin symmetric mean.

\section{Data Availability}

The Java implementation code of all quantitative comparison methods and related data used to support the findings of this study have been deposited in the GitHub repository (https://github.com/YuchuChingQin/AOsOfqROFNs).

\section{Conflicts of Interest}

The authors declare that there are no conflicts of interest regarding the publication of this paper.

\section{Acknowledgments}

The authors would like to acknowledge the financial supports by the National Natural Science Foundation of China (nos. 51765012 and 61562016) and the Key Laboratory Project of Guangxi (no. GIIP1805).

\section{Supplementary Materials}

The supplementary material associated with the present paper consists of ten sections: Appendixes A-J, which provide the proofs of Theorems 1-10, respectively. (Supplementary Materials)

\section{References}

[1] S. Greco, J. Figueira, and M. Ehrgott, Multiple Criteria Decision Analysis: State of the Art Surveys, Springer-Verlag New York, New York, USA, 2016.

[2] H. Bustince, E. Barrenechea, M. Pagola et al., "A historical account of types of fuzzy sets and their relationships," IEEE 
Transactions on Fuzzy Systems, vol. 24, no. 1, pp. 179-194, 2016.

[3] L. A. Zadeh, "Fuzzy sets," Information and Control, vol. 8, no. 3, pp. 338-353, 1965.

[4] K. T. Atanassov, "Intuitionistic fuzzy sets," Fuzzy Sets and Systems, vol. 20, no. 1, pp. 87-96, 1986.

[5] S. K. De, R. Biswas, and A. R. Roy, "Some operations on intuitionistic fuzzy sets," Fuzzy Sets and Systems, vol. 114, no. 3, pp. 477-484, 2000.

[6] E. B. Jamkhaneh and H. Garg, "Some new operations over the generalized intuitionistic fuzzy sets and their application to decision-making process," Granular Computing, vol. 3, no. 2, pp. 111-122, 2018.

[7] P. Liu and S.-M. Chen, "Group decision making based on Heronian aggregation operators of intuitionistic fuzzy numbers," IEEE Transactions on Cybernetics, vol. 47, no. 9, pp. 2514-2530, 2017.

[8] P. Liu, J. Liu, and S.-M. Chen, "Some intuitionistic fuzzy Dombi Bonferroni mean operators and their application to multi-attribute group decision making," Journal of the Operational Research Society, vol. 69, no. 1, pp. 1-24, 2018.

[9] H. Garg and D. Rani, "Some generalized complex intuitionistic fuzzy aggregation operators and their application to multicriteria decision-making process," Arabian Journal for Science and Engineering, vol. 44, no. 3, pp. 2679-2698, 2019.

[10] Z. Zhang and W. Pedrycz, "Models of mathematical programming for intuitionistic multiplicative preference relations," IEEE Transactions on Fuzzy Systems, vol. 25, no. 4, pp. 945-957, 2017.

[11] F. Meng, J. Tang, and H. Fujita, "Linguistic intuitionistic fuzzy preference relations and their application to multi-criteria decision making," Information Fusion, vol. 46, pp. 77-90, 2019.

[12] Q. Lei and $\mathrm{Z}$. $\mathrm{Xu}$, "Chain and substitution rules of intuitionistic fuzzy calculus," IEEE Transactions on Fuzzy Systems, vol. 24, no. 3, pp. 519-529, 2016.

[13] Z. Ai and Z. Xu, "Multiple definite integrals of intuitionistic fuzzy calculus and isomorphic mappings," IEEE Transactions on Fuzzy Systems, vol. 26, no. 2, pp. 670-680, 2018.

[14] H. Garg, "Novel intuitionistic fuzzy decision making method based on an improved operation laws and its application," Engineering Applications of Artificial Intelligence, vol. 60, pp. 164-174, 2017.

[15] P. Liu and X. Liu, "Multiattribute group decision making methods based on linguistic intuitionistic fuzzy power Bonferroni mean operators," Complexity, vol. 2017, Article ID 3571459, 15 pages, 2017.

[16] J. Xu, J. Dong, S. Wan, D. Yang, and Y. Zeng, "A heterogeneous multiattribute group decision-making method based on intuitionistic triangular fuzzy information," Complexity, vol. 2019, Article ID 9846582, 18 pages, 2019.

[17] R. R. Yager, "Pythagorean membership grades in multicriteria decision making," IEEE Transactions on Fuzzy Systems, vol. 22, no. 4, pp. 958-965, 2014.

[18] R. R. Yager, "Properties and applications of pythagorean fuzzy sets," in Imprecision and Uncertainty in Information Representation and Processing, P. Angelov and S. Sotirov, Eds., vol. 332, Springer, Studies in Fuzziness and Soft Computing, pp. 119-136, Springer, 2016.

[19] S. Dick, R. R. Yager, and O. Yazdanbakhsh, "On Pythagorean and complex fuzzy set operations," IEEE Transactions on Fuzzy Systems, vol. 24, no. 5, pp. 1009-1021, 2016.

[20] T. Y. Chen, "An effective correlation-based compromise approach for multiple criteria decision analysis with pythagorean fuzzy information," Journal of Intelligent \& Fuzzy Systems, vol. 35, no. 3, pp. 3529-3541, 2018.

[21] Z. Li and M. Lu, "Some novel similarity and distance measures of pythagorean fuzzy sets and their applications," Journal of Intelligent \& Fuzzy Systems, vol. 37, no. 2, pp. 1781-1799, 2019.

[22] X. Peng, "New similarity measure and distance measure for Pythagorean fuzzy set," Complex \& Intelligent Systems, vol. 5, no. 2, pp. 101-111, 2019.

[23] X. T. Nguyen, V. D. Nguyen, V. H. Nguyen, and H. Garg, "Exponential similarity measures for pythagorean fuzzy sets and their applications to pattern recognition and decisionmaking process," Complex \& Intelligent Systems, vol. 5, no. 2, pp. 217-228, 2019.

[24] W. Yang, J. Shi, Y. Liu, Y. Pang, and R. Lin, "Pythagorean fuzzy interaction partitioned bonferroni mean operators and their application in multiple-attribute decision-making," Complexity, vol. 2018, Article ID 3606245, 25 pages, 2018.

[25] R. Zhang, J. Wang, X. Zhu, M. Xia, and M. Yu, "Some generalized Pythagorean fuzzy Bonferroni mean aggregation operators with their application to multiattribute group decision-making," Complexity, vol. 2017, Article ID 5937376, 16 pages, 2017.

[26] W. Yang and Y. Pang, "New pythagorean fuzzy interaction maclaurin symmetric mean operators and their application in multiple attribute decision making," IEEE Access, vol. 6, pp. 39241-39260, 2018.

[27] M. S. A. Khan, S. Abdullah, A. Ali, and F. Amin, "Pythagorean fuzzy prioritized aggregation operators and their application to multi-attribute group decision making," Granular Computing, vol. 4, no. 2, pp. 249-263, 2019.

[28] P. Ren, Z. Xu, and X. Gou, "Pythagorean fuzzy TODIM approach to multi-criteria decision making," Applied Soft Computing, vol. 42, pp. 246-259, 2016.

[29] T. Y. Chen, "Remoteness index-based Pythagorean fuzzy VIKOR methods with a generalized distance measure for multiple criteria decision analysis," Information Fusion, vol. 41, pp. 129-150, 2018.

[30] D. Liang, Z. Xu, D. Liu, and Y. Wu, "Method for three-way decisions using ideal TOPSIS solutions at Pythagorean fuzzy information," Information Sciences, vol. 435, pp. 282-295, 2018.

[31] R. R. Yager, "Generalized orthopair fuzzy sets," IEEE Transactions on Fuzzy Systems, vol. 25, no. 5, pp. 1222-1230, 2017.

[32] X. Shu, Z. Ai, Z. Xu, and J. Ye, "Integrations of q-rung orthopair fuzzy continuous Information," IEEE Transactions on Fuzzy Systems, vol. 27, no. 10, pp. 1974-1985, 2019.

[33] J. Gao, Z. Liang, J. Shang, and Z. Xu, "Continuities, derivatives and differentials of q-rung orthopair fuzzy functions," IEEE Transactions on Fuzzy Systems, vol. 27, no. 8, pp. 1687-1699, 2019.

[34] R. R. Yager and N. Alajlan, "Approximate reasoning with generalized orthopair fuzzy sets," Information Fusion, vol. 38, pp. 65-73, 2017.

[35] B. P. Joshi, A. Singh, P. K. Bhatt, and K. S. Vaisla, "Interval valued q-rung orthopair fuzzy sets and their properties," Journal of Intelligent \& Fuzzy Systems, vol. 35, no. 5, pp. 5225-5230, 2018.

[36] S. Sharma and S. Singh, "On some generalized correlation coefficients of the fuzzy sets and fuzzy soft sets with application in cleanliness ranking of public health centres," Journal of Intelligent \& Fuzzy Systems, vol. 36, no. 4, pp. 3671-3683, 2019. 
[37] P. Wang, J. Wang, G. Wei, and C. Wei, "Similarity measures of q-rung orthopair fuzzy sets based on cosine function and their applications," Mathematics, vol. 7, no. 4, p. 340, 2019.

[38] X. Peng, J. Dai, and H. Garg, "Exponential operation and aggregation operator for q-rung orthopair fuzzy set and their decision-making method with a new score function," International Journal of Intelligent Systems, vol. 33, no. 11, pp. 2255-2282, 2018.

[39] Y. Xing, R. Zhang, Z. Zhou, and J. Wang, "Some q-rung orthopair fuzzy point weighted aggregation operators for multi-attribute decision making," Soft Computing, 2019.

[40] P. Liu and P. Wang, "Some q-rung orthopair fuzzy aggregation operators and their applications to multiple-attribute decision making," International Journal of Intelligent Systems, vol. 33, no. 2, pp. 259-280, 2018.

[41] P. Liu and J. Liu, "Some q-rung orthopair fuzzy Bonferroni mean operators and their application to multi-attribute group decision making," International Journal of Intelligent Systems, vol. 33, no. 2, pp. 315-347, 2018.

[42] P. Liu and P. Wang, "Multiple-attribute decision making based on Archimedean Bonferroni operators of q-rung orthopair fuzzy numbers," IEEE Transactions on Fuzzy Systems, vol. 27, no. 5, pp. 834-848, 2019.

[43] Z. Liu, P. Liu, and X. Liang, "Multiple attribute decisionmaking method for dealing with heterogeneous relationship among attributes and unknown attribute weight information under q-rung orthopair fuzzy environment," International Journal of Intelligent Systems, vol. 33, no. 9, pp. 1900-1928, 2018.

[44] W. Yang and Y. Pang, "New q-rung orthopair fuzzy partitioned Bonferroni mean operators and their application in multiple attribute decision making," International Journal of Intelligent Systems, vol. 34, no. 3, pp. 439-476, 2019.

[45] G. Wei, H. Gao, and Y. Wei, "Some q-rung orthopair fuzzy Heronian mean operators in multiple attribute decision making," International Journal of Intelligent Systems, vol. 33, no. 7, pp. 1426-1458, 2018.

[46] Z. Liu, S. Wang, and P. Liu, "Multiple attribute group decision making based on q-rung orthopair fuzzy Heronian mean operators," International Journal of Intelligent Systems, vol. 33, no. 12, pp. 2341-2363, 2018.

[47] G. Wei, C. Wei, J. Wang, H. Gao, and Y. Wei, "Some q-rung orthopair fuzzy Maclaurin symmetric mean operators and their applications to potential evaluation of emerging technology commercialization," International Journal of Intelligent Systems, vol. 34, no. 1, pp. 50-81, 2019.

[48] P. Liu, S. M. Chen, and P. Wang, "Multiple-attribute group decision-making based on q-rung orthopair fuzzy power Maclaurin symmetric mean operators," IEEE Transactions on Systems, Man, and Cybernetics: Systems, 2018.

[49] K. Bai, X. Zhu, J. Wang, and R. Zhang, "Some partitioned Maclaurin symmetric mean based on q-rung orthopair fuzzy information for dealing with multi-attribute group decision making," Symmetry, vol. 10, no. 9, p. 383, 2018.

[50] J. Wang, R. Zhang, X. Zhu, Z. Zhou, X. Shang, and W. Li, "Some q-rung orthopair fuzzy Muirhead means with their application to multi-attribute group decision making," Journal of Intelligent \& Fuzzy Systems, vol. 36, no. 2, pp. 1599-1614, 2019.

[51] M. Grabisch, J. L. Marichal, R. Mesiar, and E. Pap, Encyclopedia of Mathematics and its Applications 127, Aggregation Functions, Cambridge University Press, Cambridge, UK, 2009.
[52] M. Grabisch, J.-L. Marichal, R. Mesiar, and E. Pap, “Aggregation functions: means," Information Sciences, vol. 181, no. 1, pp. 1-22, 2011.

[53] M. Grabisch, J.-L. Marichal, R. Mesiar, and E. Pap, “Aggregation functions: construction methods, conjunctive, disjunctive and mixed classes," Information Sciences, vol. 181, no. 1, pp. 23-43, 2011.

[54] R. F. Muirhead, "Some methods applicable to identities and inequalities of symmetric algebraic functions of n letters," Proceedings of the Edinburgh Mathematical Society, vol. 21, pp. 144-162, 1902.

[55] P. Liu and D. Li, "Some Muirhead mean operators for intuitionistic fuzzy numbers and their applications to group decision making," PLoS One, vol. 12, no. 1, Article ID e0168767, 2017.

[56] J. Zhu and Y. Li, "Pythagorean fuzzy Muirhead mean operators and their application in multiple-criteria group decision-making," Information, vol. 9, no. 6, p. 142, 2018.

[57] E. P. Klement, R. Mesiar, and E. Pap, Triangular Norms, Kluwer Academic Publishers, Dordrecht, Netherlands, 2000.

[58] G. Wei, "Some induced geometric aggregation operators with intuitionistic fuzzy information and their application to group decision making," Applied Soft Computing, vol. 10, no. 2, pp. 423-431, 2010. 


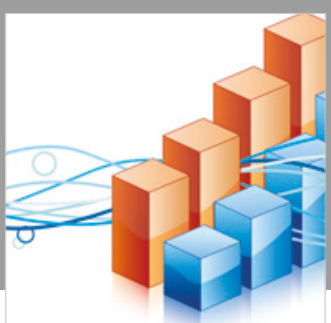

Advances in

Operations Research

\section{-n-m}
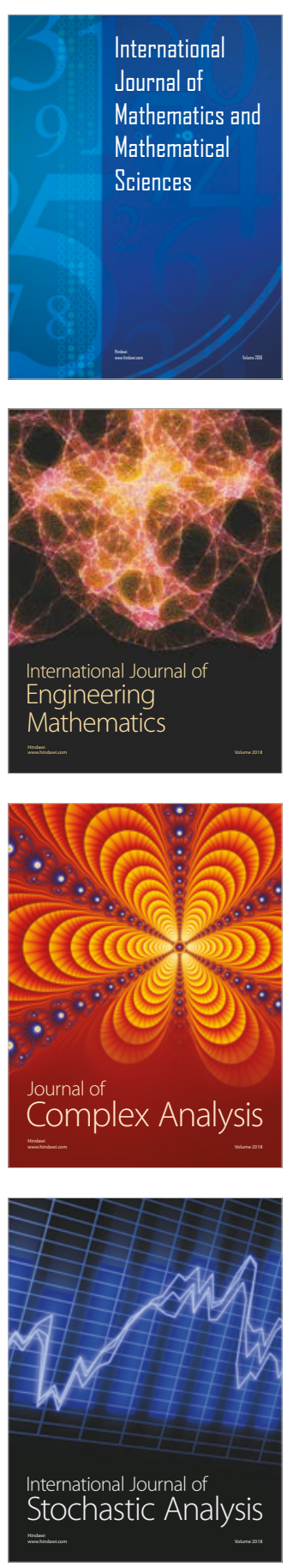
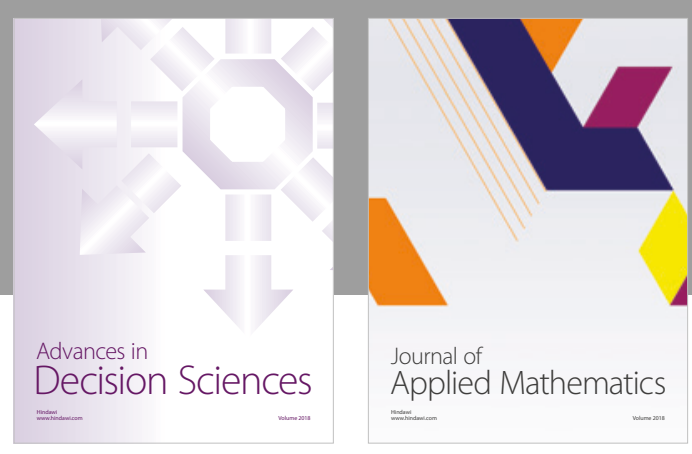

Journal of

Applied Mathematics
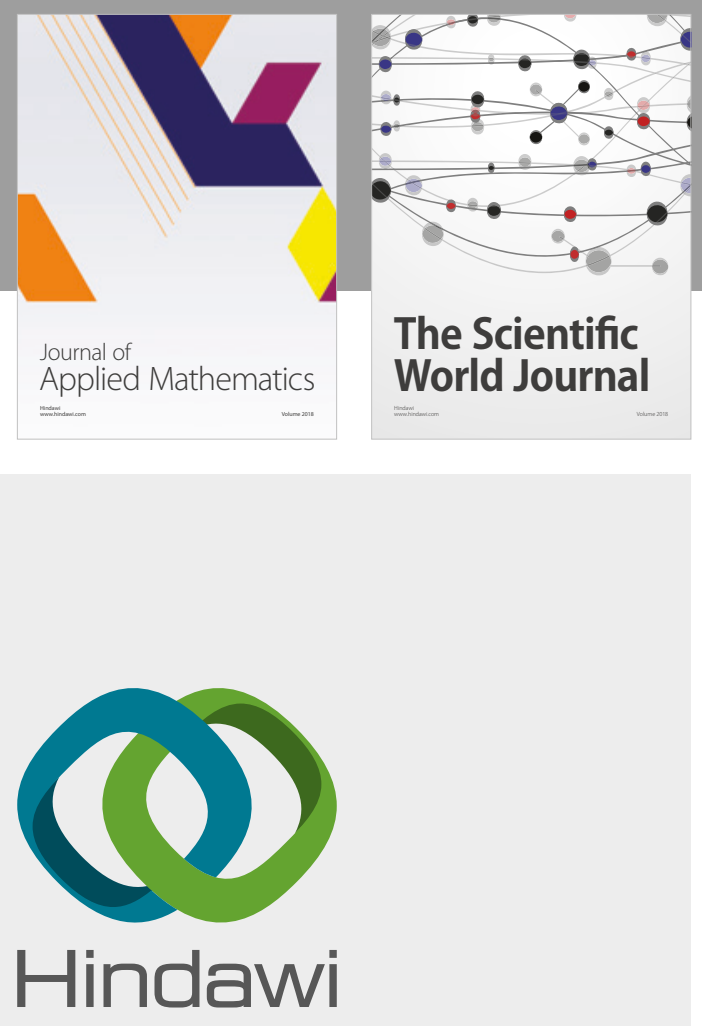

Submit your manuscripts at

www.hindawi.com

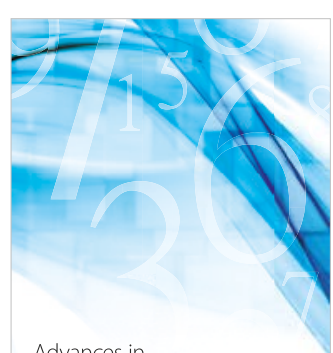

Advances in
Numerical Analysis
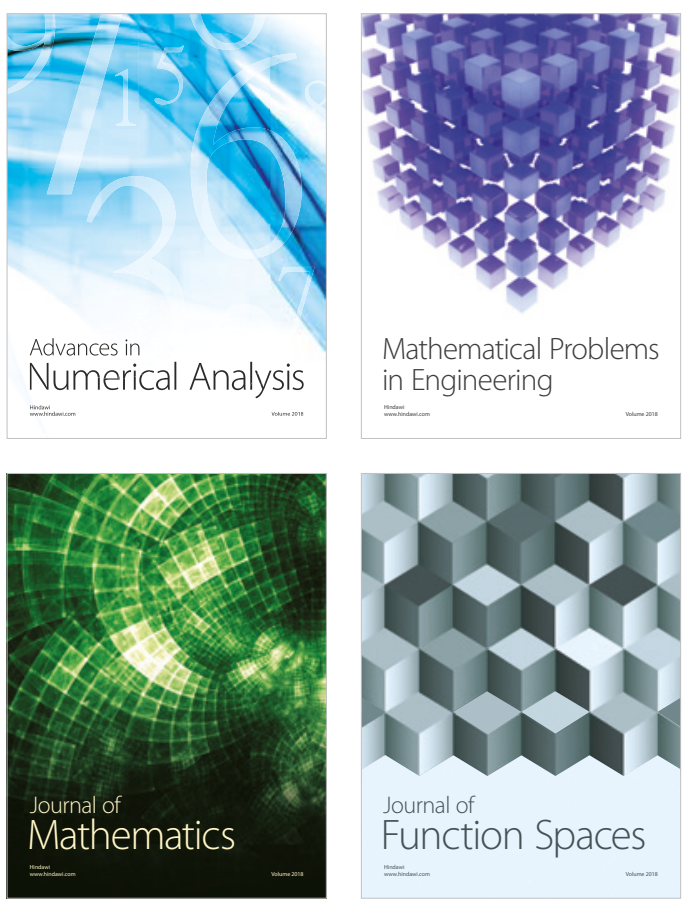

Mathematical Problems in Engineering

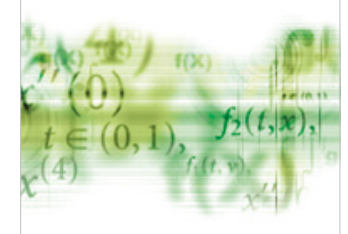

International Journal of

Differential Equations

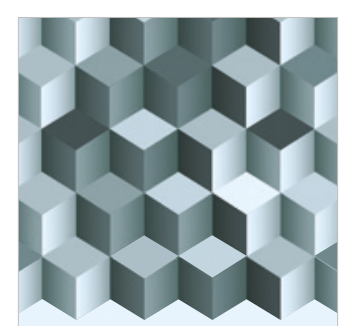

Journal of

Function Spaces
The Scientific

World Journal

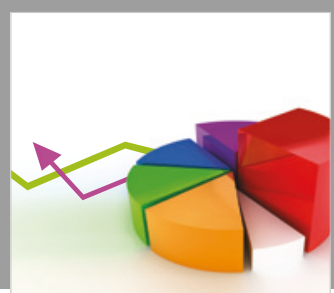

Journal of

Probability and Statistics
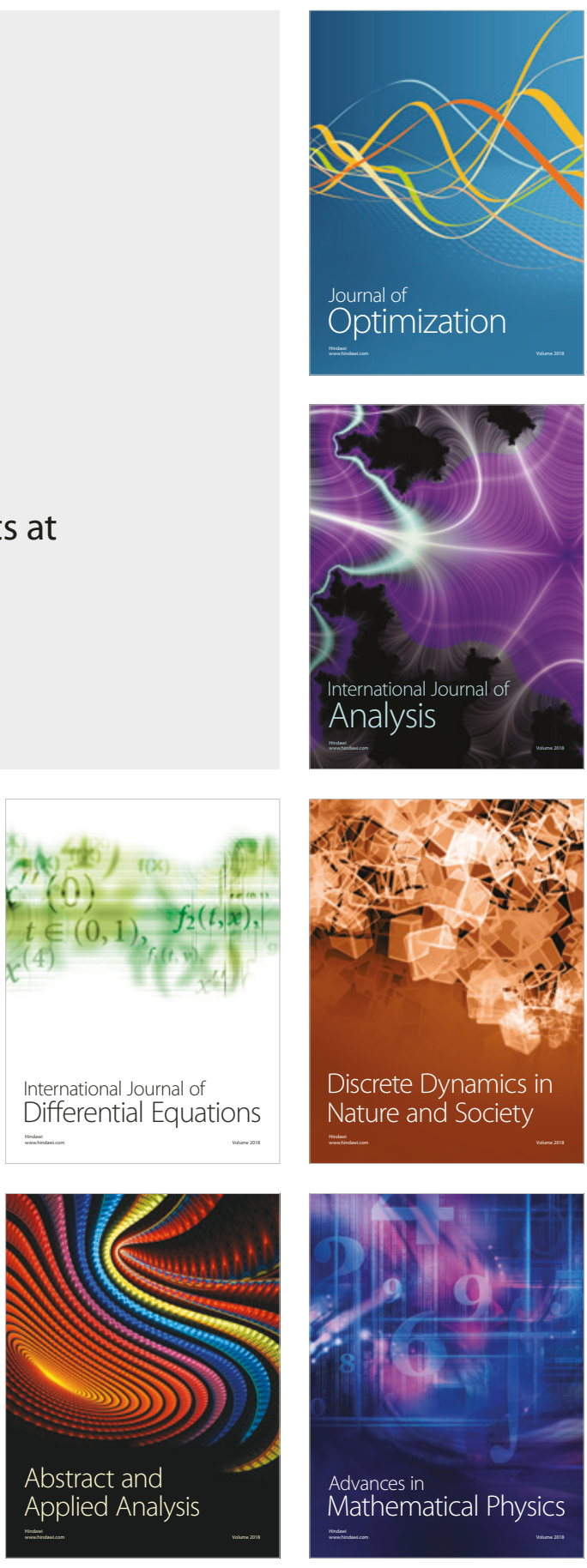